\title{
Competitive Pricing Strategies in Social Networks*
}

\author{
Ying-Ju Chen ${ }^{\dagger} \quad$ Yves Zenou ${ }^{\ddagger} \quad$ Junjie Zhou ${ }^{\S}$
}

March 12, 2018

\begin{abstract}
We study pricing strategies of competing firms that sell heterogeneous products to consumers in a social network. Goods are substitutes and there are network externalities between neighboring consumers. We show that there exists a unique subgame perfect equilibrium where, in the first stage, firms choose prices while, in the second stage, individuals consume differentiated goods. In equilibrium, firms price discriminate based on the network positions and charge lower prices to more central consumers. We also show that, under some conditions, firms' equilibrium profits decrease when either the network becomes denser or network effects increase. In contrast, consumers always benefit from being more connected to each other because this increases their utilities and, at the same time, this lowers the prices due to intensified competition between firms. We determine the optimal network structure both for firms and consumers, and compare uniform pricing and discriminatory pricing from the perspectives of firms and consumers.
\end{abstract}

Keywords: social networks, pricing, competition, differentiated products, welfare, network design.

JEL classifications: L14, L13, D43, D85

${ }^{*}$ We would like to thank the editor, three anonymous referees, Rabah Amir, Masaki Aoyagi, Francis Bloch, Ozan Candogan, Yi-Chun Chen, Yongmin Chen, Gabrielle Demange, Itay Fainmesser, Jacob Goeree, Ben Golub, Ben Hermalin, Bruno Jullien, Alessandro Lizzeri, Alessandro Pavan, Cheng-Zhong Qin, John Quah, Andrew Rhodes, Chris Shannon, Adam Szeidl, Satoru Takahashi, Guofu Tan, Curtis Taylor, Julian Wright, Jidong Zhou and conference and seminar participants at Stockholm University, Singapore Management University, the Johns Hopkins Carey Business School, National University of Singapore, University of Technology Sydney, University of Southern California, CUHK, SHUFE, UIBE, 2014 Workshop on Industrial Organization and Management Strategy (The University of Hong Kong), 2015 POMS-HK Conference at Guangzhou, and 2017 AMES for valuable comments. The usual disclaimers apply.

${ }^{\dagger}$ School of Business and Management \& School of Engineering, The Hong Kong University of Science and Technology; e-mail: imchen@ust.hk.

†Department of Economics, Monash University, IFN and CEPR. e-mail: yves.zenou@monash.edu.

§Department of Economics, National University of Singapore; e-mail: zhoujjo3001@gmail.com. 


\section{Introduction}

The past decade has witnessed an emerging role of social networks in shaping individual choices. Consumers now make consumption decisions largely based on whether their close friends, neighbors, and celebrities also adopt the same products. In economic terminology, these social goods provide network externalities to connected consumers. ${ }^{1}$ Various sources of network externalities have been documented in economics. For telecommunication devices, these externalities are generated via physical connections. For operating systems and software packages, the externalities come from compatibility concerns. Network externalities may also arise because people want to conform to the behavior of their peers. The successes of Facebook, Linkedin, Twitter, Whatsapp, WeChat and various online game producers have confirmed the high potential of market profitability in the social networking business.

These social interactions create abundant opportunities for service providers and thereby lead to intense competition among them. For instance, in the mobile and data services market, consumers can choose among AT\&T, Verizon, T-mobile, and other operators in the United States. In the market for mobile messaging apps, Whatsapp, Line, and WeChat are fighting for market shares. In such a scenario, consumers are confronted with various options to stay connected with their friends, and service providers strive to induce the consumers to lean towards their own products rather than their competitors' products.

To better understand these issues, we provide a framework that examines product competition between firms when products are differentiated and exhibit local network effects. Each consumer has access to two products, which are offered by two firms. Within each product there are local network externalities amongst the consumers in terms of their consumption utilities: a consumer pays more attention to her close friends' decisions than to others' choices. As in Ballester et al. (2006), we develop a model with strategic complementarities in consumption choices so that more consumption from a consumer reinforces other consumers' decisions to consume the same good. In contrast, to capture the competition between firms, the products are assumed to be substitutable. ${ }^{2}$ The firms incur heterogeneous costs of serving different consumers and are allowed to charge discriminatory prices to these consumers.

We show that there is a unique subgame perfect equilibrium where, first, firms choose the prices of each good for each consumer, and, then, the individuals decide their consumption levels of the two goods. We provide a full characterization of the equilibrium prices, which can be decomposed into two parts. The first term corresponds to the monopoly price, which is independent of the network configuration. The second term is proportional to the Katz-Bonacich centrality measure of a consumer (Katz 1953; Bonacich 1987). Thus, contrary to the monopoly case (Bloch and Quérou (2013)), the equilibrium prices exhibit strong network dependence where more central consumers obtain a larger discount because of their impact in terms of consumption

\footnotetext{
${ }^{1}$ Network externalities exist if the value of a product increases when there are more users joining the network. Here, we are focusing on local network externalities where the value of consuming a product for a given agent increases when others directly connected to this agent consume this product.

${ }^{2}$ In the Online Appendix C.3, we also consider the case of complementary products.
} 
on their neighbors.

In the real world, firms do price discriminate consumers based on their location or centrality in their networks. This is often done through digital marketing, which is an important aspect of firms' strategy. A key aspect of digital marketing is influencer marketing. ${ }^{3}$ Firms are gathering and using data on users' social media activity (social media platforms such as Facebook, Twitter, and Google+; and by consultancy firms such as Klout.com and Ammo Marketing) ${ }^{4}$ to identify influencers of a specific market segment and then orienting marketing activities around them. After acquiring this information, firms do price discriminate consumers, offering discounts to customers depending on their influence. For example, Microsoft, Sony and Samsung have influencer campaigns via Klout. In 2011, Microsoft increased its marketing efforts to promote the Windows Phone 7.5 by working with Klout and offering a free phone to users who had a high "Klout" score. More recently, highly influential Klout users received a free Sony NEX ${ }_{3} \mathrm{~N}$ camera and Sony Action Cam.

There are many other examples of price discrimination based on the degree of influence of consumers. For example, providing price discounts to communications between "friends and family" members has become an increasingly popular practice in telecommunication industry. A prominent example was MCI's Friends and Family Program, which offered price discounts to long distance communications between consumers and their pre-selected friends and family members. Sprint offered a similar plan that applied discounts automatically to those telephone numbers connected most frequently with consumers during a billing period. Both examples can be seen as price discrimination based on strength of callers' social ties (Shi 2013).

Building upon the equilibrium price discrimination in this competitive setting, we also show that increasing network externalities among consumers or having a denser network pushes the equilibrium price downwards. Indeed, stronger network effects or a denser network influence profits in two ways. On the one hand, they tend to increase firms' profits because they enhance demands due to higher utility externalities for consumers. On the other hand, they can lead to price reductions because stronger network effects or denser networks lead to fiercer competition between firms. The former effect is dominated by (dominates) the latter when products are close substitutes (sufficiently differentiated). By contrast, a monopoly firm always obtains a higher profit under the same circumstances. Therefore, competition can lead to substantially different implications in terms of prices and profits.

We find that consumers always benefit from being more connected to each other because this increases their consumption utilities and, at the same time, intensifies firms' competition. This suggests that the complete network maximizes consumer surplus. In contrast, firms may either

\footnotetext{
3Influencer marketing (also influence marketing) is a form of marketing in which the focus is placed on influential people rather than the target market as a whole. It identifies the individuals that have influence over potential buyers, and orients marketing activities around these influencers.

4For example, Klout.com provides social media analytics to firms to measure a user's influence in her social network (see Rao et al. (2015)). The service uses social network data (such as Twitter, Facebook, Google+, LinkedIn, YouTube, Instagram, and Wikipedia) and assigns individuals a "Klout" score, which presumably reflects their influence.
} 
prefer the complete network or the empty network, depending on the degree of product substitution. Finally, we compare uniform pricing and discriminatory pricing from the perspectives of firms and consumers. We show that when the network is not regular, firms obtain higher profits under uniform pricing when the products are sufficiently differentiated. In contrast, consumers are better off under uniform pricing than under discriminatory pricing when the products are highly substitutable. We articulate how these contrasting preferences arise from the firms' salient incentives to compensate or discriminate against players that are more central.

The remainder of this paper unfolds as follows. Section 2 reviews some relevant literature. Section 3 describes the model. Section 4 determines the equilibrium outcomes both in the second stage (consumers' consumption stage) and in the first stage (firms' pricing strategies), derives some comparative statics results on equilibrium prices and extends the model for the case when consumers only consume one good in equilibrium. Section 5 determines the welfare of this economy both for consumers and firms and derive some comparative statics results. Section 6 gives the optimal network structure for both consumers and firms, and examines the policy implication of uniform pricing rule. Finally, Section 7 concludes. In the Appendix, we provide the proofs of all our results in the main text. In the Online Appendix, we derive some matrix operations and define the Katz-Bonacich centrality (Online Appendix A), deal with the single representative consumer case (Online Appendix B), provide additional results for the duopoly case (Online Appendix C), illustrate our results for specific networks (Online Appendix D), characterize the total welfare of the economy (Online Appendix E), and provide the proofs of all the results in the Online Appendix (Online Appendix F).

\section{Related literature}

A large literature in economics has investigated the issue of network effects/externalities. The classical papers primarily focus on the aggregate level of network externalities (e.g., Rohlfs (1974), Katz and Shapiro (1985), Farrell and Saloner (1986)). Monopoly pricing of network goods is modeled and analyzed in various papers such as Cabral et al. (1999), Dybvig and Spatt (1983), and Ochs and Park (2010). 5 The competitive pricing problem is mostly studied in the context of two-sided networks in which players on one side care about the aggregate contributions of those on the other side (see, e.g., Armstrong (2006), Caillaud and Jullien (2003), and Rochet and Tirole (2006)). Using our terminology, this corresponds to the complete bipartite network case. In contrast, we study local network effects and explicitly model the network structure among players. ${ }^{6}$.

Ballester et al. (2006) provide a tractable approach to study network games with strategic complementarities amongst players using linear-quadratic utility functions. They show that the Nash equilibrium in effort is proportional to the "Katz-Bonacich centrality" of each agent. Two

\footnotetext{
${ }^{5}$ See Economides (1996) for an extensive survey of this literature.

${ }^{6}$ The economics of networks is a growing field. For recent overviews, see Jackson (2008), Ioannides (2012), Jackson et al. (2017) and Jackson and Zenou (2015).
} 
recent contributions by Bloch and Quérou (2013) and Candogan et al. (2012) incorporate the pricing decisions into the framework of Ballester et al. (2006). They independently show that if the monopoly firm can price discriminate among players and the network effects are symmetric (undirected), the resulting optimal prices do not depend on the network structure. Fainmesser and Galeotti (2016) use a somewhat different framework and consider the possibility that the firm knows partial information about the players' in-degrees or/and out-degrees (i.e., how influential they are or how significantly they are influenced by their neighbors). Allowing for third-degree price discrimination, Fainmesser and Galeotti (2016) show that the optimal pricing depends on the network configuration as well as the firm's knowledge about it. ${ }^{7}$

Our work is related to Ballester et al. (2006). However, contrary to Ballester et al. (2006), we introduce multiple products framework. As a result, the consumption game amongst players is not only driven by network externalities but also by the interdependence between different products. Few papers have analyzed monopoly pricing for products with network effects; these include Hartline et al. (2008), Arthur et al. (2009), Bloch and Quérou (2013), Candogan et al. (2012), and Fainmesser and Galeotti (2016). ${ }^{8}$ As stated above, when there is only one firm in the market, different researchers have shown that monopoly prices are network-independent but this is a knife-edge result that heavily depends on the quadratic utility specification and on constant marginal costs. When competition is introduced, as in our model, this is not anymore the case: firms do price discriminate even with quadratic utility function and constant marginal costs. To be more precise, we extend Bloch and Quérou (2013) and Candogan et al. (2012) by allowing price competition between firms. We show that the competitive pricing strategies can in general exhibit strong network dependence and the firms' profitability can even be reduced when the network becomes denser. When the products are independent, our model degenerates to the models of Bloch and Quérou (2013) and Candogan et al. (2012). We show that the dependence of prices on network structure would not arise in the monopoly case. While the network-dependent pricing shares some similarities with that in Fainmesser and Galeotti (2016), the underlying drivers are very different. Fainmesser and Galeotti (2016) show that, in directed networks, a monopoly firm charge different prices to distinct consumers; thus, such price heterogeneity comes from the heterogeneity in network effects. In contrast, in our model, the sole driver for networkdependent pricing strategies is product competition. In addition, we study the welfare effects for both consumers and firms and identify the optimal network structures. ${ }^{9}$

In a related paper, Aoyagi (2014) assumes that both products are incompatible and, therefore,

7There are papers that study pricing problems on social networks without the use of the linear-quadratic utility framework. Leduc et al. (2017) and Lobel et al. (2016) study pricing policies that involve price discounts as well as referral incentives. See also Shin (2017) for a related pricing problem and Bimpikis et al. (2016) and Grabisch et al. (2018), who study the optimal targeted advertising strategies and highlight their dependence on the underlying social network structure.

${ }^{8}$ See also Shi (2013), Deroïan and Gannon (2006), Billand et al. (2014), Bloch (2016), Carroni and Righi (2015), Currarini and Feri (2015), Belhaj and Deroïan (2016), Bimpikis et al. (2015), Ushchev and Zenou (2015), Zhang and Chen (2018), and Zhou and Chen (2017).

${ }^{9}$ In a recent paper, Fainmesser and Galeotti (2017) introduce competition with network effects but focus on the case when the network is unobserved while, here, we focus on the importance of substitution between goods when the network is perfectly observable. 
each consumer only consumes at most one product from two firms. With this extreme form of product substitution, the author focuses on the condition under which both firms charging marginal costs is a subgame perfect equilibrium. He also illustrates how multiplicity of equilibria in the consumption stage arises and provides some equilibrium selection criteria. In contrast, in our paper, the consumption behavior is continuous and the Nash equilibrium in the consumption stage is shown to be unique. Moreover, we allow for any degree of substitution (or complements) between products, and generate closed-form expressions on players' consumptions, equilibrium prices, and firms' profits. These analytical expressions allow us to conduct various comparative statics results on the network structure, degree of substitution, and strength of network effects.

Banerji and Dutta (2009) also consider price competition in the presence of local network effects. However, they focus on the uniform pricing case in which the firms cannot price discriminate between players. Similar assumptions on the flat/uniform pricing are imposed in Armstrong (2006), Caillaud and Jullien (2003), and Rochet and Tirole (2006) as well. As in Aoyagi (2014), they provide conditions under which either a firm monopolizes the market or the market is completely segmented. Ambrus and Argenziano (2009) and Jullien (2011) discuss the pricing competition among platforms in two-sided markets. Thus, the underlying network structure is bipartite. Fazeli and Jadbabaie (2012) consider a similar problem. However, they assume that the firms charge uniform pricing to the consumers. Moreover, in their model, each consumer has an inelastic unit demand and decides the fraction of consumptions between the two products.

\section{Model}

Consider a duopoly model where two firms $A$ and $B$ sell products to $n$ consumers, indexed by $i=1,2, \cdots, n$, who are embedded in a social network. Let $\mathcal{N}=\{1,2, \cdots, n\}$ denote the set of consumers. The network with $n$ players is described by the $n \times n$ adjacency matrix $\mathbf{G}=\left(g_{i j}\right)$ where $g_{i j}=0$ means that there is no link between $i$ and $j$ and $g_{i j}>0$ measures the intensity of the link between $i$ and $j .{ }^{10}$ We assume that there are no self loops, i.e. $g_{i i}=0$ and that $g_{i j}=g_{j i}$, i.e., $\mathbf{G}$ is symmetric. Each consumer consumes two products, $A$ and $B$, which are offered by firms $A$ and $B$, and can be consumed in any nonnegative quantities. Firm $A(B)$ is the sole provider for product $A(B)$; thus, each of them has full price discrimination power over their products. However, the two products are interdependent and there are network externalities between neighboring consumers.

\footnotetext{
${ }^{10}$ In most applications, $\mathrm{G}$ is the adjacency matrix of a network, and we abuse notation and let $\mathrm{G}$ denote both the network and the adjacency matrix.
} 
Consumers' utilities. Consumer $i^{\prime}$ s utility function can be expressed as follows: ${ }^{11}$

$$
\begin{aligned}
u_{i}= & a_{i}^{A} x_{i}^{A}+a_{i}^{B} x_{i}^{B}-\left\{\frac{1}{2}\left(x_{i}^{A}\right)^{2}+\frac{1}{2}\left(x_{i}^{B}\right)^{2}+\beta x_{i}^{A} x_{i}^{B}\right\} \\
& +\delta \sum_{j=1}^{n} g_{i j} x_{i}^{A} x_{j}^{A}+\delta \sum_{j=1}^{n} g_{i j} x_{i}^{B} x_{j}^{B} \\
& -p_{i}^{A} x_{i}^{A}-p_{i}^{B} x_{i}^{B} .
\end{aligned}
$$

This utility consists of three parts.

The first part of (1), $a_{i}^{A} x_{i}^{A}+a_{i}^{B} x_{i}^{B}-\left\{\frac{1}{2}\left(x_{i}^{A}\right)^{2}+\frac{1}{2}\left(x_{i}^{B}\right)^{2}+\beta x_{i}^{A} x_{i}^{B}\right\}$, corresponds to consumer $i^{\prime}$ 's own consumption utility and is expressed as a cost-benefit function. Parameter $a_{i}^{t}$ measures the intrinsic marginal utility of consumer $i$ for product $t=A, B$. The quadratic terms capture the decreasing marginal returns from consuming each product while the cross term $\beta x_{i}^{A} x_{i}^{B}$ describes the interconnection between products. While our analysis can be extended to general values of $\beta \in(-1,1)$, we will restrict our discussions to the special case when $\beta \in[0,1)$ so that the goods $A$ and $B$ are substitutes, i.e. $\frac{\partial^{2} u_{i}}{\partial x_{i}^{A} \partial x_{i}^{B}}=-\beta<0 .{ }^{12}$ When $\beta$ is close to 1 , the products are almost perfectly substitutable. ${ }^{13}$ When $\beta=0$, the two products are independent. As a result, $\beta$ measures the degree of substitution between the two goods or equivalently the degree of differentiation between goods $A$ and $B$. Indeed, the higher is $\beta$, the more substitutable the goods are and, thus, the less differentiated they are.

The second part of (1), $\delta \sum_{j=1}^{n} g_{i j} x_{i}^{A} x_{j}^{A}+\delta \sum_{j=1}^{n} g_{i j} x_{i}^{B} x_{j}^{B}$, captures the network externalities within each product. The intensity of the network effects is captured by $\delta \geq 0$ since a higher $\delta$ indicates that a consumer's utility depends more heavily on others' consumption decisions. When $g_{i j} \geq 0$, which indicates that consumers $i$ and $j$ are neighbors, a higher consumption of product $t=A, B$ from consumer $j$ increases by $\delta$ the marginal utility of consuming product $t=A, B$ for consumer $i$, i.e. $\frac{\partial^{2} u_{i}}{\partial x_{i}^{A} \partial x_{j}^{A}}=\frac{\partial^{2} u_{i}}{\partial x_{i}^{B} \partial x_{j}^{B}}=\delta$.

The third term in (1), $-p_{i}^{A} x_{i}^{A}-p_{i}^{B} x_{i}^{B}$, is the total expenditure on both products for consumer $i$, where $p_{i}^{A}$ and $p_{i}^{B}$ are the prices charged by firms $A$ and $B$ to consumer $i$, respectively.

Firms' profit functions. The marginal cost of producing product $t=A, B$ for consumer $i$ is denoted by $c_{i}^{t}$. Quite naturally, we assume that $a_{i}^{A}>c_{i}^{A}$ and $a_{i}^{B}>c_{i}^{B}, \forall i$. Given the prices $\mathbf{p}^{A}=\left(p_{1}^{A}, \cdots, p_{n}^{A}\right)^{\prime}$ and $\mathbf{p}^{B}=\left(p_{1}^{B}, \cdots, p_{n}^{B}\right)^{\prime}$, the consumptions $\mathbf{x}^{A}=\left(x_{1}^{A}, \cdots, x_{n}^{A}\right)^{\prime}$ and $\mathbf{x}^{B}=$

\footnotetext{
${ }^{11}$ In this utility function, it is assumed that there is an outside good, which is the numeraire good, which price is normalized to 1 so that there is a constant marginal utility to money. Indeed, denote by $x_{i}^{0}$ the level of consumption of an outside good for consumer $i$ and consider the utility function (1) without the last two terms and adding $x_{i}^{0}$. Then, the budget constraint for each consumer $i$ can be written as: $w=x_{i}^{0}+p_{i}^{A} x_{i}^{A}+p_{i}^{B} x_{i}^{B}$, where $w$ is the (common) income of each consumer. By plugging the value of $x_{i}^{0}$ from the budget constraint into this utility function, we obtain (1). See Online Appendix B.

${ }^{12}$ In the Online Appendix C.3, we deal with the case when $\beta \in(-1,0)$ so that the goods $A$ and $B$ are complements.

${ }^{13}$ Our analysis requires $\beta \in(-1,1)$ because we need $\beta$ to be, in absolute value, less than 1 (see Assumption 1 ). Otherwise, $u_{i}$ is not concave in $\mathbf{x}_{i}$. The case of perfect substitutes $(\beta=1)$ is of independent interest in the existing literature (e.g., Ambrus and Argenziano (2009) and Banerji and Dutta (2009)). Incidentally, Aoyagi (2014) studies the case of perfect substitutes in a somewhat different setup (each player makes a binary purchasing decision).
} 
$\left(x_{1}^{B}, \cdots, x_{n}^{B}\right)^{\prime}$, and the marginal costs $\mathbf{c}^{A}=\left(c_{1}^{A}, \cdots, c_{n}^{A}\right)^{\prime}$ and $\mathbf{c}^{B}=\left(c_{1}^{B}, \cdots, c_{n}^{B}\right)^{\prime}$, the profit is equal to: $\boldsymbol{\Pi}^{t}=\left\langle\mathbf{p}^{t}-\mathbf{c}^{t}, \mathbf{x}^{t}\right\rangle$ for firm $t=A, B .{ }^{14}$

Two benchmark models. Let us now discuss two benchmark models in the literature. The first benchmark model is when $\delta=0$ (no network effects) so that consumer $i$ 's utility function reduces to

$$
u_{i}=a_{i}^{A} x_{i}^{A}+a_{i}^{B} x_{i}^{B}-\left\{\frac{1}{2}\left(x_{i}^{A}\right)^{2}+\frac{1}{2}\left(x_{i}^{B}\right)^{2}+\beta x_{i}^{A} x_{i}^{B}\right\}-p_{i}^{A} x_{i}^{A}-p_{i}^{B} x_{i}^{B} .
$$

The above setup is commonly used in the industrial organization literature (Dixit 1979; Singh and Vives 1984), especially in love-for-variety models of monopolistic competition with linearquadratic utility (see e.g. Ottaviano et al. (2002); Melitz (2003); Combes et al. (2008)), where $\beta$ serves as an (inverse) measure of product differentiation. Indeed, since the network linkages disappear, the model degenerates to the case of $n$ independent representative consumers and the utility function corresponds to a standard linear-quadratic model with product differentiation for the case of two goods and $n$ consumers. As a result, the two firms engage in a price competition to attract each representative consumer. In the Online Appendix B, we provide the detailed derivations for a single representative consumer where the utility function is given by (2).

The second benchmark model is when $\beta=0$ so that consumer $i$ 's utility is now equal to

$$
u_{i}=\left\{a_{i}^{A} x_{i}^{A}-\frac{1}{2}\left(x_{i}^{A}\right)^{2}+\delta \sum_{j=1}^{n} g_{i j} x_{i}^{A} x_{j}^{A}-p_{i}^{A} x_{i}^{A}\right\}+\left\{a_{i}^{B} x_{i}^{B}-\frac{1}{2}\left(x_{i}^{B}\right)^{2}+\delta \sum_{j=1}^{n} g_{i j} x_{i}^{B} x_{j}^{B}-p_{i}^{B} x_{i}^{B}\right\} .
$$

Because the two terms in brackets are totally separable, there is no interaction between the two products. Consequently, each firm simply acts as a monopoly in her own market, and the model degenerates to the single-product monopoly setting studied by Bloch and Quérou (2013) and Candogan et al. (2012).

Assumptions. We now introduce some assumptions regarding the degree of activity interdependence and the intensity of network effects. ${ }^{15}$ Denote by $\lambda_{1}(\mathbf{G})$ the largest eigenvalue of the adjacency matrix $\mathbf{G}$.

Assumption 1. $1-|\beta|-\delta \lambda_{1}(\mathbf{G})>0$.

Assumption 1 guarantees the existence and uniqueness of equilibrium in the consumption stage. When $\beta=0$, this condition is equivalent to $\delta<1 / \lambda_{1}(\mathbf{G})$, which is commonly assumed in the network literature with a single activity (see e.g. Ballester et al. (2006) and Zhou and Chen (2015)).

The next two assumptions concern the intrinsic marginal utilities and marginal costs. To simplify exposition, in some parts of the paper, we will assume the following semi-symmetry (SS) assumption:

Assumption 2. $a_{i}^{A}=a_{i}^{B}=a_{i}, c_{i}^{A}=c_{i}^{B}=c_{i}, i=1,2, \cdots, n$.

\footnotetext{
${ }^{14}$ The inner product of two vectors $\mathbf{x}=\left(x_{1}, \cdots, x_{n}\right)^{\prime}$ and $\mathbf{y}=\left(y_{1}, \cdots, y_{n}\right)^{\prime}$ in $\mathbf{R}^{n}$ is denoted as $\langle\mathbf{x}, \mathbf{y}\rangle=\sum_{i} x_{i} y_{i}$.

${ }^{15}$ See Online Appendix A for all matrix and vector notations and some related definitions.
} 
Assumption 2 is referred to as semi-symmetry (SS) since it implies that, for each consumer $i$, the intrinsic marginal utilities and the marginal costs are the same between the two markets $A$ and $B$ but it still keep the heterogeneity of $a_{i}$ and $c_{i}$ between consumers.

Assumption 3. $a_{i}^{A}=a_{i}^{B}=a, c_{i}^{A}=c_{i}^{B}=c, i=1,2, \cdots, n$.

This assumption is referred to as full symmetry (FS) because it implies that all consumers are ex ante identical apart from their network positions.

Timing. The sequence of events is as follows. First, firms $A$ and $B$ determine simultaneously the price vectors $\mathbf{p}^{A}=\left(p_{1}^{A}, \cdots, p_{n}^{A}\right)^{\prime}$ and $\mathbf{p}^{B}=\left(p_{1}^{B}, \cdots, p_{n}^{B}\right)^{\prime}$. Second, after observing $\mathbf{p}^{A}$ and $\mathbf{p}^{B}$, each consumer $i$ non-cooperatively chooses her consumption bundle $\mathbf{x}_{i}=\left(x_{i}^{A}, x_{i}^{B}\right)$ and this choice is made simultaneously with all other consumers. The solution concept for this two-stage game is subgame-perfect Nash equilibrium (SPNE). As usual, we solve the model backwards.

\section{Equilibrium Analysis}

By backward induction, we first derive the equilibrium outcomes amongst consumers in the consumption stage. We then analyze the firms' pricing strategies.

Consumption stage. We first take as given the price vectors $\mathbf{p}^{A}$ and $\mathbf{p}^{B}$ offered by firms $A$ and $B$ and solve the consumers' equilibrium consumptions. Define the following two $n \times n$ matrices:

$$
\mathbf{M}^{+}:=\left[(1+\beta) \mathbf{I}_{n}-\delta \mathbf{G}\right]^{-1} \text {, and } \mathbf{M}^{-}:=\left[(1-\beta) \mathbf{I}_{n}-\delta \mathbf{G}\right]^{-1} \text {. }
$$

For $t=A, B$, denote $\mathbf{x}^{t}=\left(x_{1}^{t}, \cdots, x_{n}^{t}\right)^{\prime}, \mathbf{a}^{t}=\left(a_{1}^{t}, \cdots, a_{n}^{t}\right)^{\prime}$ and $\mathbf{p}^{t}=\left(p_{1}^{t}, \cdots, p_{n}^{t}\right)^{\prime}$. We have the following theorem, which is directly adapted from Chen et al. (2017), after taking prices into account:

Proposition 1. If Assumption 1 holds, then, for any prices $\mathbf{p}^{A}$ and $\mathbf{p}^{B}$ charged by firms $A$ and $B$, there exists a unique equilibrium in the consumption stage characterized by:

$$
\left\{\begin{array}{l}
\mathbf{x}^{A}=\mathbf{M}^{+}\left[\left(\frac{\mathbf{a}^{A}+\mathbf{a}^{B}}{2}\right)-\left(\frac{\mathbf{p}^{A}+\mathbf{p}^{B}}{2}\right)\right]+\mathbf{M}^{-}\left[\left(\frac{\mathbf{a}^{A}-\mathbf{a}^{B}}{2}\right)-\left(\frac{\mathbf{p}^{A}-\mathbf{p}^{B}}{2}\right)\right] \\
\mathbf{x}^{B}=\mathbf{M}^{+}\left[\left(\frac{\mathbf{a}^{A}+\mathbf{a}^{B}}{2}\right)-\left(\frac{\mathbf{p}^{A}+\mathbf{p}^{B}}{2}\right)\right]-\mathbf{M}^{-}\left[\left(\frac{\mathbf{a}^{A}-\mathbf{a}^{B}}{2}\right)-\left(\frac{\mathbf{p}^{A}-\mathbf{p}^{B}}{2}\right)\right] .
\end{array}\right.
$$

Proposition 1 provides a clean characterization of the equilibrium outcomes and has some intuitive interpretations. It characterizes the equilibrium of a quadratic game with two products where the social network is explicitly modeled. We show that the average consumption across the two goods taken by each player is determined by a social interaction matrix multiplied by the average intrinsic marginal utilities minus average prices across the two goods while (half of) the difference between consumptions in two goods is determined by another social-interaction matrix times (half of) the difference in intrinsic marginal utilities minus difference in prices between the two products. 
Moreover, Proposition 1 leads to the following characterizations:

$$
\overline{\mathbf{x}}=\mathbf{M}^{+}(\overline{\mathbf{a}}-\overline{\mathbf{p}}) \text { and } \widehat{\mathbf{x}}=\mathbf{M}^{-}(\widehat{\mathbf{a}}-\widehat{\mathbf{p}})
$$

where $\overline{\mathbf{x}}:=\left(\mathbf{x}^{A}+\mathbf{x}^{B}\right) / 2, \overline{\mathbf{a}}:=\left(\mathbf{a}^{A}+\mathbf{a}^{B}\right) / 2$ and $\overline{\mathbf{p}}:=\left(\mathbf{p}^{A}+\mathbf{p}^{B}\right) / 2$ are the average consumption, intrinsic marginal utilities and price, and $\widehat{\mathbf{x}}:=\left(\mathbf{x}^{A}-\mathbf{x}^{B}\right) / 2, \widehat{\mathbf{a}}:=\left(\mathbf{a}^{A}-\mathbf{a}^{B}\right) / 2$, and $\widehat{\mathbf{p}}:=\left(\mathbf{p}^{A}-\mathbf{p}^{B}\right) / 2$ corresponds to half of the difference in consumptions, intrinsic marginal utilities and prices. This shows that what determines the consumption of each good is the sum and difference of the marginal utility of consumption and prices of each product multiplied by two matrices $\mathbf{M}^{+}$and $\mathbf{M}^{-}$. These two matrices, which count the number of walks in $\mathbf{G}$ starting from $i$ and ending at $j$, where walks of length $k$ are weighted by $\delta^{k} /(1+\beta)^{1+k}$ and $\delta^{k} /(1-\beta)^{1+k}$, respectively (see (3)), are clearly related to the Katz-Bonacich centrality (which is defined in the Online Appendix A). They appear with a decay factor that not only depends on $\delta$, the intensity of network externalities between directly connected individuals, but also on $\beta$, the degree of substitution between the two products.

Indeed, when the consumers make their consumption decisions, they take into account both local network effects and the degree of substitution between the two products. Observe that we have assumed here a linear-quadratic utility function for the consumers, which allows us to have linear best responses and a clean characterization of the equilibrium consumptions. As shown by Bramoullé et al. (2014), our equilibrium analysis and results on optimal pricing carry over to a more general class of utility functions that induce linear best responses.

We can also obtain some comparative statics results. Since $\beta \geq 0, \mathbf{M}^{+} \preceq \mathbf{M}^{-}$. Then, it is straightforward to show that:

$$
\frac{\partial \mathbf{x}^{A}}{\partial \mathbf{a}^{A}}=-\frac{\partial \mathbf{x}^{A}}{\partial \mathbf{p}^{A}}=\frac{\mathbf{M}^{+}+\mathbf{M}^{-}}{2} \succ \mathbf{0}, \text { and } \frac{\partial \mathbf{x}^{B}}{\partial \mathbf{a}^{A}}=-\frac{\partial \mathbf{x}^{B}}{\partial \mathbf{p}^{A}}=\frac{\mathbf{M}^{+}-\mathbf{M}^{-}}{2} \preceq \mathbf{0}
$$

Indeed, when $\mathbf{p}^{A}\left(\mathbf{p}^{B}\right)$, the price of product $A(B)$, increases, each consumer consumes less of product $A(B)$ and more of product $B(A)$ (pure substitution effect). These comparative statics formulas have implications for the equilibrium prices determined in the next subsection.

Let us now impose a symmetry condition to obtain a cleaner expression.

Corollary 1. Suppose that Assumptions 1 and 2 hold, which mean that $\mathbf{a}^{A}=\mathbf{a}^{B}=\mathbf{a}$. Suppose that both firms charge the same prices, i.e., $\mathbf{p}^{A}=\mathbf{p}^{B}=\mathbf{p}$. Then, $\mathbf{x}^{A}=\mathbf{x}^{B}=\mathbf{M}^{+}(\mathbf{a}-\mathbf{p})$.

Pricing stage. In Proposition 1, for given prices, we characterized the equilibrium consumption bundles for all consumers in the social network. In this section, we solve the first stage of the game, i.e. the pricing decisions of both firms. For $t=A, B$, denote $c^{t}=\left(c_{1}^{t}, \cdots, c_{n}^{t}\right)^{\prime}$. The profit of each firm is given by: $\Pi^{A}(\mathbf{p})=\left\langle\mathbf{p}^{A}-\mathbf{c}^{A}, \mathbf{x}^{A}\left(\mathbf{p}^{A}, \mathbf{p}^{B}\right)\right\rangle$ and $\Pi^{B}(\mathbf{p})=\left\langle\mathbf{p}^{B}-\mathbf{c}^{B}, \mathbf{x}^{B}\left(\mathbf{p}^{A}, \mathbf{p}^{B}\right)\right\rangle$, where $\mathbf{x}^{A}\left(\mathbf{p}^{A}, \mathbf{p}^{B}\right)$ and $\mathbf{x}^{B}\left(\mathbf{p}^{A}, \mathbf{p}^{B}\right)$ are given in Proposition 1 .

Theorem 1. Suppose Assumptions 1 and 2 hold. Then, there exists a unique interior equilibrium in the 
pricing stage where both firms charge $\mathbf{p}^{* A}=\mathbf{p}^{* B}=\mathbf{p}^{*}$, given by:

$$
\mathbf{p}^{*}=\left(\frac{\mathbf{a}+\mathbf{c}}{2}\right)-\frac{\beta}{2}\left[(2-\beta) \mathbf{I}_{n}-2 \delta \mathbf{G}\right]^{-1}(\mathbf{a}-\mathbf{c}) .
$$

First, observe that we use the semi-symmetry (SS) Assumption 2 in Theorem 1, which implies that, for each consumer $i$, the intrinsic marginal utilities and the marginal costs are the same between the two markets $A$ and $B$ but there is still heterogeneity of $a_{i}$ and $c_{i}$ between consumers. In Theorem C.2, which is stated in the Online Appendix C.4, we solve the general case when Assumption 2 is relaxed so that the equilibrium prices are not the same anymore. We still obtain a similar result since each price $\mathbf{p}^{A *}$ and $\mathbf{p}^{B *}$ can be expressed as Katz-Bonacich centralities.

In the symmetric equilibrium characterized by Theorem 1, firm A (or B) has no incentive to deviate from $\mathbf{p}^{*}$. In other words, the marginal benefit due to higher price-cost margins and the marginal cost due to reduced demands must cancel out at the equilibrium, i.e.,

$$
\langle\underbrace{\mathbf{M}^{+}\left(\mathbf{a}-\mathbf{p}^{*}\right)}_{=\mathbf{x}^{\mathbf{A}}}, \Delta \mathbf{p}^{* A}\rangle=\langle\underbrace{\left(\frac{\mathbf{M}^{+}+\mathbf{M}^{-}}{2}\right) \Delta \mathbf{p}^{* A}}_{=\Delta \mathbf{x}^{\mathbf{A}}}, \mathbf{p}^{*}-\mathbf{c}\rangle,
$$

which holds for any price change $\Delta \mathbf{p}^{* A} \in \mathbf{R}^{n}$. Equation (8) leads to the following equation: ${ }^{16}$

$$
\mathbf{M}^{+}\left(\mathbf{a}-\mathbf{p}^{*}\right)=\frac{1}{2}\left(\mathbf{M}^{+}+\mathbf{M}^{-}\right)\left(\mathbf{p}^{*}-\mathbf{c}\right),
$$

which, after simplification, leads to (7) in Theorem 1.

Second, let us give some economic intuition of the equilibrium price vector $\mathbf{p}^{*}$ given in Theorem 1. This equilibrium price is made of two terms. The first one, $(\mathbf{a}+\mathbf{c}) / 2$, is the monopoly price, i.e. the equilibrium price when products are independent $(\beta=0)$. Moreover, by Lemma C.I in Online Appendix C.2, it is also the collusive price (or the equilibrium price without competition). The second term in (7) is due to competition. In particular, it is easily verified that

$$
\frac{\beta}{2}\left[(2-\beta) \mathbf{I}_{n}-2 \delta \mathbf{G}\right]^{-1}(\mathbf{a}-\mathbf{c})=\frac{\beta}{2(2-\beta)} \mathbf{b}\left(\mathbf{G}, \frac{2 \delta}{2-\beta}, \mathbf{a}-\mathbf{c}\right),
$$

where $\mathbf{b}\left(\mathbf{G}, \frac{2 \delta}{2-\beta}, \mathbf{a}-\mathbf{c}\right)$ is the vector of Katz-Bonacich centralities (defined in the Online Appendix A) for network $\mathbf{G}$, where the discount factor is $2 \delta /(2-\beta)$ and the weight is a $-\mathbf{c}$. Because $0 \leq \beta<1$, this term is always positive: with competition the business stealing effect always drives prices downwards. More importantly, this term is consumer specific. ${ }^{17}$

\footnotetext{
${ }^{16}$ Here we have implicitly used the fact that both $\mathbf{M}^{+}$and $\mathbf{M}^{-}$are symmetric, which is indeed the case since $\mathbf{G}$ is symmetric. When $\mathrm{G}$ is not symmetric, the equilibrium characterization for the consumption stage (i.e., Proposition 4) still holds. Furthermore, we still have a unique equilibrium price in the first stage. The equilibrium pricing is pinned down by equation (9), except that we need to put a transpose sign on the matrix $\left(\mathbf{M}^{+}+\mathbf{M}^{-}\right)$in equation (9). The expression for $\mathbf{p}^{*}$ is thus slightly more complicated than the current form in (7).

${ }_{17}$ When the network effect $\delta$ is moderate, the second term in equation (10) is dominated by the first term $(\mathbf{a}+\mathbf{c}) / 2$,
} 
Finally, observe that in Corollary 1 , we give the values of the consumption of each good when Assumptions 1 and 2 hold. These values could be negative if the price of these goods were too high. Since the price of each good is given in Theorem 1 , let us verify that the equilibrium consumptions are always strictly positive. Since, by Corollary $1, \mathbf{x}^{A}=\mathbf{x}^{B}=\mathbf{x}=\mathbf{M}^{+}(\mathbf{a}-\mathbf{p})$, we need to show that: $\mathbf{a}>\mathbf{p}$, which, using (7), is equivalent to:

$$
(\mathbf{a}-\mathbf{c})+\beta\left[(2-\beta) \mathbf{I}_{n}-2 \delta \mathbf{G}\right]^{-1}(\mathbf{a}-\mathbf{c})>\mathbf{0}
$$

which is clearly always true. So, the consumption of the two goods will always be strictly positive in equilibrium.

Implications in terms of price discrimination. Because different consumers obtain different price adjustments, depending on $\beta, \delta$ and $\mathbf{G}$, in equilibrium, firms do exercise price discrimination. Indeed, since the second term in (7) (see (10)) is proportional to the Katz-Bonacich centrality measure of each consumer, the more central the consumers are in the network, the lower is the price paid for consuming goods $A$ and $B$. This is an interesting result, which shows that more central consumers obtain a larger discount because of their impact in terms of consumption on their neighbors. In other words, when firms have a precise information about "who influences whom" in the network, they can set different prices to different consumers in order to enhance network effects and earn larger profits. The most central agents receive each product at a lower price while others, less central, are exploited by the firms and pay a higher price.

Monopoly versus duopoly pricing. Let us now give some intuition of why network effects matter only where there is competition between firms. In the monopoly case (without competition), given a fixed price vector $\mathbf{p}$, the demands are equal to: $\mathbf{x}^{m}=\mathbf{M}\left(\mathbf{a}-\mathbf{p}^{m}\right)$, where $\mathbf{M}=[\mathbf{I}-\delta \mathbf{G}]^{-1}$ (Bloch and Quérou 2013; Candogan et al. 2012). Hence, the monopoly firm's pricing problem is $\max _{\mathbf{p}^{m}}\left\langle\mathbf{x}^{m}, \mathbf{p}^{m}-\mathbf{c}\right\rangle$. If $\mathbf{p}^{m *}$ is the optimal monopoly price vector, then, after each price deviation, $\mathbf{p}^{* m}$ will be equal to $\mathbf{p}^{* m}-\boldsymbol{\Delta} \mathbf{p}^{* m}$. Thus, the marginal cost (lower margins) and marginal benefit (demand enhancing effect) must exactly cancel each other, i.e.,

$$
\langle\underbrace{\mathbf{M}\left(\mathbf{a}-\mathbf{p}^{* m}\right)}_{=\mathbf{x}^{* \mathbf{m}}}, \Delta \mathbf{p}^{* m}\rangle=\langle\underbrace{\mathbf{M} \Delta \mathbf{p}^{* m}}_{=\Delta \mathbf{x}^{* \mathbf{m}}}, \mathbf{p}^{* m}-\mathbf{c}\rangle,
$$

which holds for any price changes $\Delta \mathbf{p}^{* m}$. As a consequence,

$$
\mathbf{M}\left(\mathbf{a}-\mathbf{p}^{* m}\right)=\mathbf{M}\left(\mathbf{p}^{* m}-\mathbf{c}\right)
$$

which leads to the monopoly price:

$$
\mathbf{p}^{* m}=\frac{\mathbf{a}+\mathbf{c}}{2} .
$$

The price $\mathbf{p}^{* m}$ is independent of any network effect and, in particular, of the position of consumers in the network. Indeed, when selling network goods to a group of consumers, a firm faces

and hence the equilibrium prices are positive. In the extreme case when $\delta=0, \mathbf{p}^{*}=[\mathbf{a}(1-\beta)+\mathbf{c}] /(2-\beta)$, which is obviously positive. 
two trade-offs when choosing optimal prices, regardless of whether there is competition or not. On the one hand, more central players obtain more network externalities from their neighbors. Hence, the firm has a strong incentive to charge a larger premium to these consumers in order to capture the surplus associated with it. This is the usual logic of third-degree price discrimination. On the other hand, the firm has an incentive to give these consumers larger discounts because they generate large network externalities on their neighbors. This is the logic of internalization of externalities in network games.

For a monopoly firm, these two forces exactly cancel each other out, and thus the firm charges a price of $(\mathbf{a}+\mathbf{c}) / 2$, which is independent of the network. ${ }^{18}$ This is clearly an artefact of the linear demand structure. However, when competing with another firm, firm $A^{\prime}$ s incentives to attract consumers towards her own products is stronger. Indeed, because the two goods are substitute, more consumption of product $A$ leads automatically to less consumption of product $B$. This reduction is further reinforced by the complementarity effects within each product, which, in turn, makes product $A$ even more attractive.

Moreover, we can compare equations (9) and (12). We observe that the difference between the monopoly and the competitive cases is purely driven by the difference between the matrices $\mathbf{M}^{+}$and $\left(\mathbf{M}^{+}+\mathbf{M}^{-}\right) / 2$, which matters only when products are not independent, i.e. when $\beta \neq 0$ (if $\beta=0, \mathbf{M}^{+}=\mathbf{M}^{-}=\mathbf{M}$ ). In fact, $\mathbf{M}^{-} \succeq \mathbf{M}^{+}$when $\beta>0$. Thus, in (9), the right-hand side is greater than the left-hand side when setting $\mathbf{p}^{*}=\mathbf{p}^{* m}=(\mathbf{a}+\mathbf{c}) / 2$. Therefore, under the symmetry price $\mathbf{p}^{*}=(\mathbf{a}+\mathbf{c}) / 2$, firm $A$ has a strict incentive to undercut her prices $\mathbf{p}^{A}$, and so does firm $B$. Consequently, the symmetric equilibrium prices with competition must be lower than $\mathbf{p}^{*}=(\mathbf{a}+\mathbf{c}) / 2 .{ }^{19}$ The exact differences correspond to the adjustment term given by (7) in Theorem 1. It is easily verified that, when either $\beta$ or $\delta$ increases, the adjustment term increases and thus, with a higher degree of product substitution or stronger network effects, the equilibrium prices decrease.

Collusive price. In the Online Appendix C.2, we analyze the case where the two firms determine the prices $\mathbf{p}^{A}$ and $\mathbf{p}^{B}$ jointly to maximize their total profits. This could be the case when two firms merge into a single firm that controls both prices. We show that, again, the equilibrium prices (collusive prices) do not depend on the network structure.

Complementary versus substitutable goods. In the Online Appendix C.3, we analyze the case where the two goods are complement $(\beta<0)$ instead of substitute $(\beta>0)$. In Proposition C.1, we show that, when $\beta<0$, the competitive prices $\mathbf{p}^{*}$ now exhibit influence-based premium so that more central consumers in the network are charged a higher price than less central consumers. This is exactly the opposite result to that obtained in Theorem 1 . This is because, when products are complements, when the price of good $A$ increases, then individuals will consume less of good $B$ while, when products are substitutes, they consume more of good $B$. Therefore, the slope of the prices with respect to centrality is related to the nature of product interdependence. This

\footnotetext{
${ }^{18}$ See Bloch (2016) for further discussions regarding this network-independent result in the monopoly case.

${ }^{19}$ For complementary products $(\beta<0)$, the incentive to undercut the price is reversed. As a consequence, the equilibrium price is actually higher than $\mathbf{p}^{* m}=(\mathbf{a}+\mathbf{c}) / 2$.
} 
may be potentially empirically testable.

Special cases. We now apply Theorem 1 to the two benchmark cases presented in Section 3.

When $\delta=0$ (no network effect), there is a unique equilibrium given by:

$$
\mathbf{p}^{*}=\frac{(1-\beta) \mathbf{a}+\mathbf{c}}{2-\beta}
$$

which is decreasing in $\beta$, and converges to the marginal cost $\mathbf{c}$ (Bertrand prices) when $\beta$ approaches 1 .

The other special case $(\beta=0)$ exhibits very peculiar properties. The equilibrium price is characterized in the next Corollary.

Corollary 2. Suppose Assumptions 1 and 2 hold.

(i) When $\beta=0$ (two independent networks), $\mathbf{p}^{*}=\mathbf{p}^{* m}=(\mathbf{a}+\mathbf{c}) / 2$, which is independent of the parameter $\delta$ and the network matrix $\mathbf{G}$.

(ii) For a fixed nonempty network $\mathbf{G}$, if $\mathbf{p}^{*}$ is independent of $\delta$, then it must be the case that $\beta=0$.

(iii) For any pair of consumers $i, j$ with $g_{i j}>0$, suppose $\delta>0$ and consumer $i$ 's equilibrium price $p_{i}$ is independent of $a_{j}$ or $c_{j}$. Then it must be the case that $\beta=0$.

Note that item (i) in Corollary 2 revisits the established results in the literature (Bloch and Quérou (2013) and Candogan et al. (2012)), and we present it here for completeness. When $\beta=0$ (the two products are independent), each firm behaves as a monopolist in her own product market. Therefore, the problem degenerates to the monopoly setup and the intensity of network effects $\delta$ has no impact on the optimal (equilibrium) prices. This result is very different to the one obtained in Theorem 1 for the duopoly case where both the structure of the network and the intensity of network effects matter in the price determination of the products. In other words, the competition between firms make the impact of network effects on prices non negligible.

Corollary 2 also shows that this surprising result crucially depends on the product independence assumption or the monopoly assumption. In the presence of product competition, the network does matter. In addition, it also provides the identification result in the reverse direction. Unless the products are independent (i.e., unless the two firms have no interactions), the equilibrium prices must utilize the knowledge of the network structure. Taken together, Corollary 2 attempts to identify the boundary within which the insight of network-independent optimal pricing applies.

Small network effects. In the Online Appendix C.1, we consider an asymptotic regime with sufficiently small network effects, i.e., when $\delta$ is sufficiently small. Using Taylor expansions, we obtain more transparent expressions of the equilibrium prices, which only uses information about of the degrees of consumers. 
Comparative statics analysis on prices. Let us now derive some comparative statics results on the equilibrium prices.

Definition 1. $\mathbf{G}^{\prime} \succ \mathbf{G}$ if $\mathbf{G}^{\prime}$ contains $\mathbf{G}$ as a subgraph and there are additional links in $\mathbf{G}^{\prime}$ but not in $\mathbf{G}$. In that case, network $\mathbf{G}^{\prime}$ is denser than network $\mathbf{G}$.

This provides an incomplete ordering of graphs and we can compare two graphs if the sets of links are nested. Clearly $\lambda_{1}\left(\mathbf{G}^{\prime}\right) \geq \lambda_{1}(\mathbf{G})$ if $\mathbf{G}^{\prime} \succ \mathbf{G}$.

Proposition 2. Suppose Assumptions 1 and 2 hold for both $\mathbf{G}$ and $\mathbf{G}^{\prime}$.

1. We have:

$$
\begin{gathered}
\frac{\partial p_{i}^{*}}{\partial a_{i}}>0, \forall i \text { and } \frac{\partial p_{i}^{*}}{\partial a_{j}}<0 \text { when } i \neq j, \\
\frac{\partial p_{i}^{*}}{\partial c_{j}} \geq 0, \forall(i, j),
\end{gathered}
$$

and

$$
\frac{\partial p_{i}^{*}}{\partial \delta} \leq 0, \forall i \text { and } \frac{\partial p_{i}^{*}}{\partial \beta} \leq 0, \forall i
$$

2. If $\mathbf{G}^{\prime} \succ \mathbf{G}$, then $\mathbf{p}^{*}\left(\mathbf{G}^{\prime}\right) \preceq \mathbf{p}^{*}(\mathbf{G})$.

The comparative statics results obtained in this proposition can be better understood using the decomposition formula (7). Recall that

$$
p_{j}^{*}=\frac{a_{j}+c_{j}}{2}-\frac{\beta}{2(2-\beta)} b_{j}\left(\mathbf{G}, \frac{2 \delta}{2-\beta},(\mathbf{a}-\mathbf{c})\right) .
$$

When $a_{j}$ increases, $p_{j}^{*}$ is affected in two different ways. First, $\left(a_{j}+c_{j}\right) / 2$ increases. Second, the other term also increases since the weight of $(\mathbf{a}-\mathbf{c})$ in the Katz-Bonacich centrality measure raises. Thus $\frac{\partial p_{i}^{*}}{\partial a_{i}}>0$. By contrast, for consumer $i \neq j, \frac{\partial p_{i}^{*}}{\partial a_{j}}<0$.

Similarly, when the cost $c_{j}$ for firm $j$ increases, $p_{j}^{*}$ goes up. Furthermore, this cost increase for consumer $j$ also negatively affects all other consumers via their social interactions. Formally, the Katz- Bonacich centrality $b_{i}$ not only depends on player i's characteristic $a_{i}-c_{i}$ but also on all the other players' characteristics. When $c_{i}$ goes up, all the $b_{j}$ s are reduced and, therefore, all of the prices $p_{i}^{*}$ rise. For player $i$, the increment is stronger as the first term also becomes larger.

When two products become more substitutable (i.e., $\beta$ increases), the firms are competing more intensively in the pricing game: the coefficient $\frac{\beta}{2(2-\beta)}$ goes up as well as the decay factor $\frac{2 \delta}{2-\beta}$ in $b_{j}\left(\mathbf{G}, \frac{2 \delta}{2-\beta},(\mathbf{a}-\mathbf{c})\right)$. Therefore, in equilibrium, both firms give more generous discounts to players, which leads to uniformly lower prices. The same intuition applies for $\delta$, the intensity of network effects. Finally, when the network becomes denser, $b_{j}\left(\mathbf{G}, \frac{2 \delta}{2-\beta},(\mathbf{a}-\mathbf{c})\right)$ becomes higher and thus prices decrease. 
In the Online Appendix D, we illustrate our main results about pricing with specific network structures. In the Online Appendix D.1, we consider the case of a dyad (complete network with two consumers); in the Online Appendix D.2, we consider the family of regular graphs such as, for example, the circle; in the Online Appendix D.3, we study the complete bipartite graph. For all these different graphs, we determine the equilibrium prices and derive some comparative statics results. Finally, in the Online Appendix D.4, we illustrate the last result of Proposition 2 by showing that switching from a star network with three consumers to a complete network with three consumers, the network becomes denser and the price of the latter network is lower for all consumers compared to that of the former network.

Consuming one good only. Let us now develop an alternative model where each consumer finds it optimal to consume one good only. The other components of this alternative model is similar to our main model. There are two firms $A$ and $B$ producing two different goods competing for $n$ consumers located in the network $\mathrm{G}$. Each player $i \in \mathcal{N}$ makes a binary decision of whether or not buying the good $A$ or $B$, i.e. $\eta_{i} \in\{A, B\}$. The utility of consumer $i$ is given by:

$$
u_{i}\left(\eta_{i}, \eta_{-i}\right)= \begin{cases}v_{i}^{A}+\phi \sum_{j} g_{i j} 1_{\left\{\eta_{j}=A\right\}}-p_{i}^{A}+\epsilon_{i}^{A} & \text { if } \eta_{i}=A \\ v_{i}^{B}+\delta \sum_{j} g_{i j} 1_{\left\{\eta_{j}=B\right\}}-p_{i}^{B}+\epsilon_{i}^{B} & \text { if } \eta_{i}=B\end{cases}
$$

where $v_{i}^{k}$ is the intrinsic valuation of each product $k=A, B$ for each consumer $i \in \mathcal{N}$, $p_{i}^{k}$ is the price charged by firm $k$ to consumer $i, 1_{\left\{\eta_{j}=k\right\}}$ is the indicator function, and $\epsilon_{i}^{k}$ is the random shocks. As above, $\delta$ uniformly scales the network effects. This is alternative model using a discrete choice approach, which generalizes Bloch and Quérou (2013) for the case of the choice of two goods. Unlike our model above, each consumer consumes either product $A$ or product $B$, but not both.

For simplicity, we assume that $\epsilon_{i}^{A}-\epsilon_{i}^{B}$ is uniformly distributed on the interval $[-t, t] .{ }^{20}$ Here $t$ measures the degree of differentiation between the two products. The timing of the game is the same as before. We focus on a symmetric setting with $v_{i}^{A}=v_{i}^{B}, \forall i$. Also, for simplicity, we normalize the marginal costs to be zero, i.e., $c_{i}=0$. Denote by $d_{i}$ the degree (i.e. number of links) of consumer $i$.

Proposition 3. Assume that $t-\delta \lambda_{1}(\mathbf{G})>0$. Then, if the utility of consumers is given by (13), there exists a unique subgame perfect Nash equilibrium where, in the first stage, both firms charge the same prices $\mathbf{p}^{*}=\left(p_{1}^{*}, \cdots, p_{n}^{*}\right)$ where $p_{i}^{*}=t-\delta d_{i}$, for each consumer $i$, and in the second state the equilibrium market demand is equal to: $\frac{1}{2} \mathbf{1}_{n}$. Moreover, in equilibrium, each firm obtains a profit of $\sum_{i \in \mathcal{N}} \frac{1}{2}\left(t-\delta d_{i}\right)$.

We show that the demand function derived from our discrete choice model with the difference of shocks being uniformly distributed is basically the same as the one derived from our benchmark model. As a result, the equilibrium prices derived from both models have the same flavor in that firms discriminate consumers based on their position in the network. To be more precise, in both models, the demand functions are both linear in prices, and the equilibrium

${ }^{20}$ This is equivalent to the Hotelling specification of product differentiation. 
prices are network dependent. The price discount in the discrete-choice model is much simpler since it is negatively related to degree of each consumer while, in our benchmark model, it was negatively related to Bonacich centrality of each consumer, which is approximately equal to the consumer's degree only when the network effects are very small (i.e. when $\delta$ close to 0 ).

Observe that, one limitation of the discrete-choice model, is that the total demand is always equal to one, and the products are always assumed to be substitutable. On the contrary, in our benchmark model, the total demand varies with price and the two products $A$ and $B$ can be either substitutes (positive $\beta$ ) or complements (negative $\beta$ ). This generates different implications. For example, it is easily checked that, when the network becomes denser, the equilibrium profit under the discrete choice model always decreases since the equilibrium demand is fixed $\left(\frac{1}{2} \mathbf{1}_{n}\right.$ by Proposition 3) and equilibrium prices decrease. On the contrary, we show in Proposition 7 below, that, in our benchmark model, the equilibrium profit could increase or decrease depending on the value of $\beta$.

\section{Welfare Analysis}

In the Online Appendix E (Theorem E.1), we determine the equilibrium consumptions $\mathbf{x}^{*}(\mathbf{G} ; \beta, \delta)$, the equilibrium profits $\boldsymbol{\Pi}^{*}(\mathbf{G} ; \beta, \delta)$, the consumer surplus $\mathbf{C S}^{*}(\mathbf{G} ; \beta, \delta)$ and the total welfare $\mathbf{T W}^{*}(\mathbf{G} ; \beta, \delta)$.

Comparative statics analysis: the consumers' perspective. Define ${ }^{21}$

$$
\phi^{E X}(\mathbf{G} ; \beta, \delta):=\frac{\left[\mathbf{I}_{n}-\delta \mathbf{G}\right]}{\left[(1+\beta) \mathbf{I}_{n}-\delta \mathbf{G}\right]\left[(2-\beta) \mathbf{I}_{n}-2 \delta \mathbf{G}\right]}
$$

Proposition 4. Suppose Assumptions 1 and 2 hold. Then,

$$
\begin{gathered}
\frac{\partial \mathbf{x}^{*}(\mathbf{G} ; \beta, \delta)}{\partial \mathbf{a}}=-\frac{\partial \mathbf{x}^{*}(\mathbf{G} ; \beta, \delta)}{\partial \mathbf{c}}=\phi^{E X}(\mathbf{G} ; \beta, \delta) \succeq \mathbf{0} \\
\frac{\partial \mathbf{x}^{*}(\mathbf{G} ; \beta, \delta)}{\partial \beta}=\frac{\partial \phi^{E X}(\mathbf{G} ; \beta, \delta)}{\partial \beta}(\mathbf{a}-\mathbf{c}) \\
\frac{\partial \mathbf{x}^{*}(\mathbf{G} ; \beta, \delta)}{\partial \delta}=\frac{\partial \phi^{E X}(\mathbf{G} ; \beta, \delta)}{\partial \delta}(\mathbf{a}-\mathbf{c}) \succeq \mathbf{0}
\end{gathered}
$$

Furthermore, if $\mathbf{G}^{\prime} \succ \mathbf{G}$, then $\mathbf{x}^{*}\left(\mathbf{G}^{\prime} ; \beta, \delta\right) \succeq \mathbf{x}^{*}(\mathbf{G} ; \beta, \delta)$.

${ }^{21}$ Note that $\phi^{E X}(\mathbf{G} ; \beta, \delta)$ should be interpreted as

$$
\left[\mathbf{I}_{n}-\delta \mathbf{G}\right] \times\left[(1+\beta) \mathbf{I}_{n}-\delta \mathbf{G}\right]^{-1} \times\left[(2-\beta) \mathbf{I}_{n}-2 \delta \mathbf{G}\right]^{-1} .
$$

Since the matrices $\left[\mathbf{I}_{n}-\delta \mathbf{G}\right],\left[(1+\beta) \mathbf{I}_{n}-\delta \mathbf{G}\right]^{-1}$ and $\left[(2-\beta) \mathbf{I}_{n}-2 \delta \mathbf{G}\right]^{-1}$ commute, the order of multiplications does not matter. Similar explanations for $\phi^{C S}(\mathbf{G} ; \beta, \delta)$ (consumer surplus) and $\phi^{P T}(\mathbf{G} ; \beta, \delta)$ (firm profit) below. Also, see Remark 1 after Theorem E.1. 
To understand this result, observe that, in equilibrium,

$$
\mathbf{x}^{*}=\underbrace{\left[(1+\beta) \mathbf{I}_{n}-\delta \mathbf{G}\right]^{-1}}_{\text {social multiplier }} \underbrace{\left(\mathbf{a}-\mathbf{p}^{*}\right)}_{\text {price effect }}
$$

Therefore, the impact of the parameters $\mathbf{a}, \mathbf{c}, \beta, \delta$ and of the network structure $\mathbf{G}$ on the equilibrium consumption is determined through the social multiplier $\mathbf{M}^{+}=\left[(1+\beta) \mathbf{I}_{n}-\delta \mathbf{G}\right]^{-1}$ and the price effect $\mathbf{a}-\mathbf{p}^{*}$. For $\mathbf{a}$ and $\mathbf{c}$, the results are straightforward since both a and $\mathbf{c}$ only affect the price effect. Let us analyze the effect of the other variables. When the products are more substitutable (i.e. $\beta$ increases), on the one hand, the equilibrium price $\mathbf{p}^{*}$ decreases (see Proposition 2), but, on the other hand, the social multiplier effect is smaller (paths are less discounted in the network). Hence, the net effect is ambiguous and depends on the relative importance of these two effects. However, when $\delta$, the importance of network effects, increases, the social multiplier is higher, and, at the same time, the equilibrium price is lower due to intensified competition (Proposition 2). This leads to an increase in consumption of the two goods. The same intuition applies for the last result on denser networks.

The aggregate consumer surplus, determined in Theorem E.1 in the Online Appendix E, is given by:

$$
C S^{*}=(1+\beta) \sum_{i}\left(x_{i}^{*}\right)^{2}=(1+\beta)\left\langle\mathbf{x}^{*}, \mathbf{x}^{*}\right\rangle
$$

Therefore, for a fixed degree of substitution $(\beta)$, the consumer surplus increases if and only the equilibrium consumption goes up. Hence, the comparative statics results of the consumer surplus are similar to that of $x^{*}$, given in Proposition 4. More precisely, define

$$
\phi^{C S}(\mathbf{G} ; \beta, \delta):=(1+\beta)\left[\frac{\left[\mathbf{I}_{n}-\delta \mathbf{G}\right]}{\left[(1+\beta) \mathbf{I}_{n}-\delta \mathbf{G}\right]\left[(2-\beta) \mathbf{I}_{n}-2 \delta \mathbf{G}\right]}\right]^{2} .
$$

We have the following result:

Corollary 3. In equilibrium, we find that:

- $\frac{\partial \mathbf{C S}^{*}(\mathbf{G} ; \beta, \delta)}{\partial \beta}=\left\langle(\mathbf{a}-\mathbf{c}), \frac{\partial \phi^{C S}(\mathbf{G} ; \beta, \delta)}{\partial \beta}(\mathbf{a}-\mathbf{c})\right\rangle$. In general, the sign of $\frac{\partial \mathbf{C S}^{*}(\mathbf{G} ; \beta, \delta)}{\partial \beta}$ is ambiguous.

- $\frac{\partial \mathbf{C S}^{*}(\mathbf{G} ; \beta, \delta)}{\partial \delta}=\left\langle(\mathbf{a}-\mathbf{c}), \frac{\partial \phi^{C S}(\mathbf{G} ; \beta, \delta)}{\partial \delta}(\mathbf{a}-\mathbf{c})\right\rangle$. Moreover $\frac{\partial \mathbf{C S}^{*}(\mathbf{G} ; \beta, \delta)}{\partial \delta} \succeq \mathbf{0}$.

- If $\mathbf{G}^{\prime} \succ \mathbf{G}$, then $\mathbf{C S}^{*}\left(\mathbf{G}^{\prime} ; \beta, \delta\right) \succeq \mathbf{C S}^{*}(\mathbf{G} ; \beta, \delta)$.

For example, as shown in (14), the network connectivity affects the consumer surplus through two different channels. The first channel is directly tied to the functional form that displays strategic complementarities and positive externalities. Given this functional form, players tend to consume more in a more connected network, holding prices fixed. The second channel operates through strategic effects, which only exist because of firms' competition. In a competitive environment, both firms reduce prices more aggressively in a denser network and this enhances 
consumer demands. Since both effects move in the same direction, the consumer surplus always increases with network connectivity. The same result holds under the monopoly case, though the second channel does not exist. We can follow the same argument to examine the comparative statics for $\beta$ and $a$ and link them to the two aforementioned effects.

Comparative statics analysis: the firms' perspective. Let us first study the impact of $\beta$ and $\delta$ on the equilibrium profit $\mathbf{\Pi}^{*}(\mathbf{G} ; \beta, \delta)$.

Define:

$$
\phi^{P T}(\mathbf{G} ; \beta, \delta):=\frac{\left[\mathbf{I}_{n}-\delta \mathbf{G}\right]\left[(1-\beta) \mathbf{I}_{n}-\delta \mathbf{G}\right]}{\left[(1+\beta) \mathbf{I}_{n}-\delta \mathbf{G}\right]\left[(2-\beta) \mathbf{I}_{n}-2 \delta \mathbf{G}\right]^{2}}
$$

We have the following result:

Proposition 5. Suppose Assumptions 1 and 2 hold. Then,

$$
\begin{gathered}
\frac{\partial \boldsymbol{\Pi}^{*}(\mathbf{G} ; \beta, \delta)}{\partial \beta}=\left\langle(\mathbf{a}-\mathbf{c}), \frac{\partial \phi^{P T}(\mathbf{G} ; \beta, \delta)}{\partial \beta}(\mathbf{a}-\mathbf{c})\right\rangle<0, \\
\frac{\partial \boldsymbol{\Pi}^{*}(\mathbf{G} ; \beta, \delta)}{\partial \delta}=\left\langle(\mathbf{a}-\mathbf{c}), \frac{\partial \phi^{P T}(\mathbf{G} ; \beta, \delta)}{\partial \delta}(\mathbf{a}-\mathbf{c})\right\rangle .
\end{gathered}
$$

To understand this result, observe that the equilibrium profit can be written as follows:

$$
\boldsymbol{\Pi}^{*}=\langle\underbrace{\left(\mathbf{p}^{*}-\mathbf{c}\right)}_{\text {price-cost margin }}, \overbrace{\underbrace{\left.(1+\beta) \mathbf{I}_{n}-\delta \mathbf{G}\right]^{-1}}_{\text {social multiplier }} \underbrace{\left(\mathbf{a}-\mathbf{p}^{*}\right)}_{\text {effective marginal utility }}}^{=\overbrace{}^{*}}\rangle .
$$

As a result, when products are more substitutable (i.e. $\beta$ increases), the equilibrium price $\mathbf{p}^{*}$ becomes lower because of fiercer competition (Proposition 2). Therefore, $\mathbf{p}^{*}-\mathbf{c}$ decreases while $\mathbf{a}-\mathbf{p}^{*}$ increases. Furthermore, paths are discounted at a lower rate and the social multiplier effects, captured by $\mathbf{M}^{+}$, are smaller. It turns out that the negative effects dominate the positive one and thus the total effect of $\beta$ on equilibrium profits is negative. By the same logic, increasing $\delta$ will affect the equilibrium profit through the same three effects identified above but in the opposite way. Indeed a higher $\delta$ leads to a higher $\mathbf{p}^{*}$ and stronger social multiplier effects. The net effect cannot, however, be signed. ${ }^{22}$

Since, in Proposition 5, we could not sign the impact of $\delta$ on equilibrium profit $\Pi^{*}$, in order to obtain more intuition, let us now consider regular graphs. ${ }^{23}$ Indeed, suppose that the adjacency matrix $\mathbf{G}$ is regular with degree $d$, i.e., $\mathbf{G} \mathbf{1}_{n}=d \mathbf{1}_{n}$, and assume, for simplicity, that $a_{i}=1$ and $c_{i}=0, \forall i$.

Proposition 6. Suppose Assumptions 1 and 3 hold and consider regular networks of degree d. Then,

\footnotetext{
${ }^{22}$ Since the total welfare consists of two parts, that is consumer surplus and profit, the comparative statics results for total welfare directly follow from those of the consumer surplus and of the firm's profit. Hence, we omit them for conciseness.

${ }^{23}$ In graph theory, a regular graph is a graph where each node or vertex has the same number of neighbors, i.e. every node has the same degree or valency.
} 
1. the equilibrium profit is always decreasing in $\beta$ :

$$
\frac{\partial \Pi^{*}(\mathbf{G} ; \beta, \delta)}{\partial \beta}<0
$$

2. Moreover, the profit is increasing in $\delta$ or $d$ if and only if $\beta$ is below a cutoff. Specifically, we have

$$
\frac{\boldsymbol{\Pi}^{*}(\mathbf{G} ; \beta, \delta)}{\partial \delta} \geq 0 \Leftrightarrow \frac{\Pi^{*}(\mathbf{G} ; \beta, \delta)}{\partial d} \geq 0 \Leftrightarrow \beta \leq \beta^{*}(1-d \delta)
$$

where $\beta^{*} \approx 0.596$ is the unique real root of $\beta^{3}+3 \beta-2 .^{24}$

Let us better understand the impact of $\delta$ (or $d$ the number of links of each consumer) on the equilibrium profit. First, for a fixed price vector, increasing $\delta$ (or $d$ ) leads to a higher intensity of the network effects because paths are discounted at a higher rate and, as a result, the consumers consume more since they are more influenced by their neighbors. This has a positive effect on profits. Second, increasing $\delta$ (or $d$ ) leads to a fiercer price competition and thus lower prices, which reduces the firms' profits. In Proposition 6, we show that the net effect depends on the value of $\beta$, the degree of substitution between products, $\delta$, the intensity of network effects and $d$, the degree of each consumer. If $\delta$ and $d$ are fixed, then when products are sufficiently highly (not too) differentiated so that $\beta$ is small (high) enough, the network effect dominates (is dominated by) the price effect and $\delta$ has a positive (negative) impact on equilibrium profits.

Observe that, for a monopoly firm, the price effect disappears because $p^{m}=(a+c) / 2$, which does not vary with $\delta$. As a result, when $\beta=0$ (monopoly case), the condition $\beta<\beta^{*}(1-$ $d \delta$ ) is trivially satisfied for any $\delta$. Therefore, increasing $\delta$ is always beneficial for a monopoly firm, but it could be detrimental in the presence of product competition.

We can summarize our findings as follows:

Observation 1. Suppose Assumptions 1 and 3 hold and consider regular networks of degree d:

1. When $\beta=0$ (which is equivalent to the monopoly case), increasing $\delta$ or increasing $d$ always benefits the firms in terms of profits.

2. Consider the duopoly case (i.e. $\beta>0$ ):

2a. When $0<\beta<\beta^{*}(1-d \delta)$, increasing $\delta$ or d improves firms' profits. $2 b$ When $\beta>\beta^{*}(1-d \delta)$, increasing $\delta$ or d reduces firms' profits.

Let us provide some graphic illustrations of these results. For $d=2$, in Figure 1 , we plot the range for which $\Pi^{*}$ is increasing or decreasing in $\delta$. In Figure 1 , the horizontal axis corresponds

\footnotetext{
${ }^{24}$ The cubic polynomial $\beta^{3}+2 \beta-2$ is strictly increasing in $\beta$ and it has a unique real root given by $\beta^{*}=\sqrt[3]{1+\sqrt{2}}-$ $\frac{1}{\sqrt[3]{1+\sqrt{2}}} \approx 0.596$.
} 
to $\beta$, while the vertical axis corresponds to $\delta$. Moreover, the dark region is when $\frac{\partial \Pi^{*}(\mathbf{G} ; \beta, \delta)}{\partial \delta}>0$ while the light region is when $\frac{\partial \Pi^{*}(\mathbf{G} ; \beta, \delta)}{\partial \delta}<0$. We see that an increase in $\beta$ (horizontal axis) and/or $\delta$ (vertical axis) can indeed lead to a decrease in equilibrium profits.

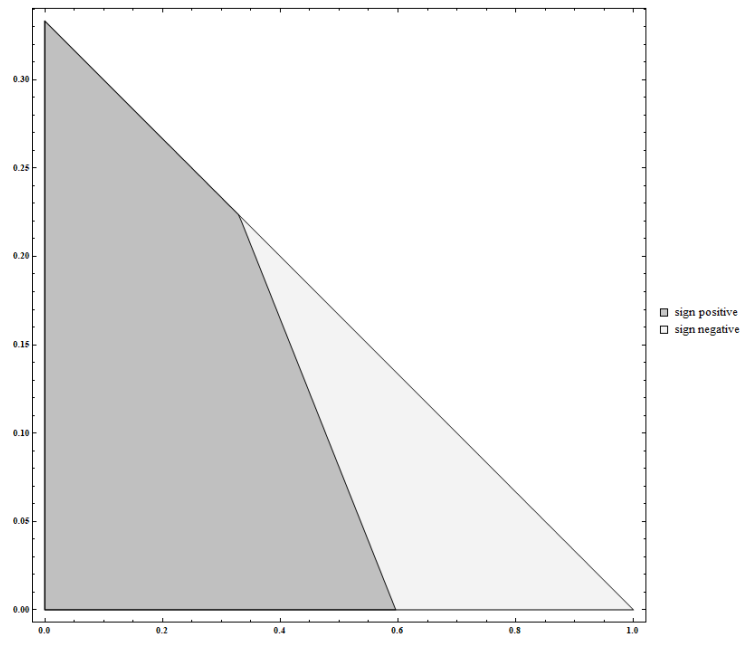

Figure 1: The sign of $\partial \Pi^{*}(\mathbf{G} ; \beta, \delta) / \partial \delta$ for a regular graph with $d=2$ : positive on the dark area, negative on the light area.

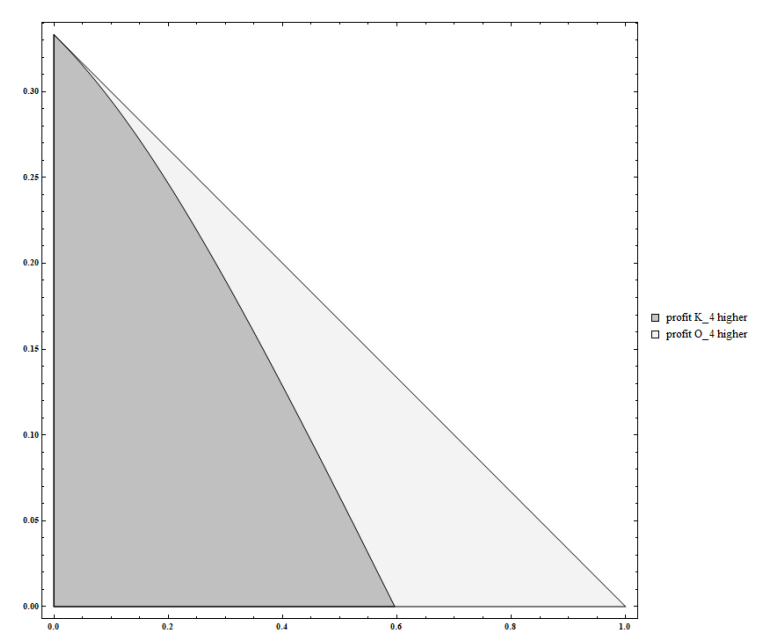

Figure 2: Comparing profits between $K_{4}$ and $\mathrm{O}_{4}$. dark area: profit of $K_{4}$ higher; light area: profit of $\mathrm{O}_{4}$ higher.

Impact of network density on equilibrium profits. Let us go back to general networks so that $\mathbf{G}$ denotes any network structure. As above (Definition 1), define a denser network $\mathbf{G}^{\prime}$ as $\mathbf{G}^{\prime} \succ \mathbf{G}$ if $\mathbf{G}^{\prime}$ contains $\mathbf{G}$ as a subgraph. We will show that the sign of $\mathbf{\Pi}^{*}\left(\mathbf{G}^{\prime} ; \beta, \delta\right)-\mathbf{\Pi}^{*}(\mathbf{G} ; \beta, \delta)$ is generally ambiguous. Let

$$
D^{\prime}=\left\{(\beta, \delta)|0 \leq \beta<1, \delta \geq 0,1-| \beta|-| \delta \mid \lambda_{1}\left(\mathbf{G}^{\prime}\right)>0\right\}
$$

be the feasible domain for the parameter $(\beta, \delta)$ under $\mathbf{G}^{\prime}$.

Proposition 7. Suppose Assumptions 1 and 2 hold. Suppose also that $\mathbf{G}^{\prime} \succ \mathbf{G}$. Then,

1. There exists a nonempty open subset $\Theta_{1}$ of $D^{\prime}$ such that for any parameters $(\beta, \delta)$ in this open set, the equilibrium profit under $\mathbf{G}^{\prime}$ is higher than that under $\mathbf{G}$ :

$$
\boldsymbol{\Pi}^{*}\left(\mathbf{G}^{\prime} ; \beta, \delta\right)>\boldsymbol{\Pi}^{*}(\mathbf{G} ; \beta, \delta), \quad \forall(\beta, \delta) \in \Theta_{1} .
$$

2. There exists a nonempty open subset $\Theta_{2}$ of $D^{\prime}$ such that for any parameters $(\beta, \delta)$ in this open set, the equilibrium profit under $\mathbf{G}^{\prime}$ is lower than that under $\mathbf{G}$ :

$$
\boldsymbol{\Pi}^{*}\left(\mathbf{G}^{\prime} ; \beta, \delta\right)<\boldsymbol{\Pi}^{*}(\mathbf{G} ; \beta, \delta), \quad \forall(\beta, \delta) \in \Theta_{2}
$$

The key message of Proposition 7 is that the equilibrium profit $\Pi^{*}(\mathbf{G} ; \beta, \delta)$ is very sensitive to the parameters of the model, in particular, $\beta$, the degree of substitution between the two products. In the proof of Proposition 7 , we show that, for small values of $\delta$, the sign of 
$\boldsymbol{\Pi}^{*}\left(\mathbf{G}^{\prime} ; \beta, \delta\right)-\boldsymbol{\Pi}^{*}(\mathbf{G} ; \beta, \delta)$ is determined by whether $\beta$ is lower or greater than $\beta^{*} \approx 0.596$. If, for example, we pick a value of $\beta=\beta_{1}\left(\beta=\beta_{2}\right)$ such that $\beta_{1}<0.596\left(\beta_{2}>0.596\right)$, then we can always find a $\delta>0$ but small enough so that $\boldsymbol{\Pi}^{*}\left(\mathbf{G}^{\prime} ; \beta, \delta\right)>\boldsymbol{\Pi}^{*}(\mathbf{G} ; \beta, \delta), \forall\left(\beta_{1}, \delta\right)$ $\left(\boldsymbol{\Pi}^{*}\left(\mathbf{G}^{\prime} ; \beta, \delta\right)<\boldsymbol{\Pi}^{*}(\mathbf{G} ; \beta, \delta), \forall\left(\beta_{2}, \delta\right)\right)$. By continuity, we obtain the result of Proposition $7 .{ }^{25}$

Let us illustrate the impact of network density on equilibrium profits using regular graphs. Let us compare the circle network $\mathbf{O}_{4}$ and the complete network $\mathbf{K}_{4}$ with four agents. Both are regular graphs with 4 consumers but they differ in their degrees since, for the circle network, the degree is two while, for the complete graph, it is three. The complete network $\mathbf{K}_{4}$ is clearly denser than the circle network $\mathbf{O}_{4}\left(\mathbf{K}_{4} \succ \mathbf{O}_{4}\right)$. This implies that the equilibrium price for the circle network is higher than for the complete network, i.e. $\mathbf{p}^{*}\left(\mathbf{K}_{4} ; \beta, \delta\right) \preceq \mathbf{p}^{*}\left(\mathbf{O}_{4} ; \beta, \delta\right)$ (Proposition 2). On the other hand, as shown in Proposition 7 , the impact on equilibrium profit is unclear. In Figure 2, we determine the profit difference between the complete network and the star with four consumers, i.e. $\boldsymbol{\Pi}^{*}\left(\mathbf{K}_{4} ; \beta, \delta\right)-\boldsymbol{\Pi}^{*}\left(\mathbf{O}_{4} ; \beta, \delta\right)$, for different values of $\beta$ (horizontal axis) and $\delta$ (vertical axis). In particular, the dark region is when $\boldsymbol{\Pi}^{*}\left(\mathbf{K}_{4} ; \beta, \delta\right)>\boldsymbol{\Pi}^{*}\left(\mathbf{O}_{4} ; \beta, \delta\right)$ while the light region is when $\boldsymbol{\Pi}^{*}\left(\mathbf{K}_{4} ; \beta, \delta\right)<\boldsymbol{\Pi}^{*}\left(\mathbf{O}_{4} ; \beta, \delta\right)$. We can see that the profit of firms is higher in $\mathbf{K}_{4}$ compared to $\mathbf{O}_{4}$ only when the parameter $\beta$ is below a certain cut-off curve of $\delta$. In particular, for very small values of $\beta$, the profits in the complete network $\mathbf{K}_{4}$ are always higher than in the circle network $\mathbf{O}_{4}$ for almost all $\delta>0$ in the feasible domain.

\section{Policy implications}

Optimal network structure. Let us now examine what is the optimal network structure for consumers and for firms. ${ }^{26}$ Let us fix the network size ( $n$ nodes) and let us allow for arbitrary number links between each pair of nodes. The set of possible network structures is denoted as $\mathcal{G}_{n}$. Let $\mathbf{E}_{n}$ denote the empty network without any link, and $\mathbf{K}_{n}$ denote the complete network. Formally, we define

$$
\begin{aligned}
\mathbf{G}^{* C S}(\beta, \delta) & :=\arg \max _{\mathbf{G} \in \mathcal{G}_{n}} \mathbf{C S}^{*}(\mathbf{G} ; \beta, \delta), \\
\mathbf{G}^{* \Pi}(\beta, \delta): & =\arg \max _{\mathbf{G} \in \mathcal{G}_{n}} \Pi^{*}(\mathbf{G} ; \beta, \delta)
\end{aligned}
$$

as the optimal network structure from the perspectives of consumers and firms, respectively. On the consumers' side, the ranking is very clear. Because Proposition 4 implies that a denser network generates lower prices, larger consumption and thus bigger consumer surplus, we obtain the following straightforward result.

\footnotetext{
${ }^{25}$ Similarly, one can show that, for a fixed graph $\mathbf{G}$, there exists a nonempty subset $\widehat{\Theta}_{1}$ of $D$ such that $\Pi^{*}$ is increasing in $\delta$ in this open set $\widehat{\Theta}_{1}$, while there exists another nonempty subset $\widehat{\Theta}_{2}$ of $D$ such that $\Pi^{*}$ is decreasing in $\delta$ in this open set $\widehat{\Theta}_{2}$.

${ }^{26}$ Very few papers have examined optimal network design in network games. Exceptions include Hiller (2017), König et al. (2016) and Belhaj et al. (2016).
} 
Theorem 2. Suppose Assumptions 1 and 2 hold. ${ }^{27}$ Then, every consumers prefers the complete network over any other network, moreover,

$$
\mathbf{G}^{* C S}(\beta, \delta)=\mathbf{K}_{n}, \quad \forall(\beta, \delta)
$$

Theorem 2 shows that consumers always benefit from being more connected to each other, i.e. the consumers' surplus is the largest in the complete network. This arises from two reasons. First, there is a direct effect since, other things being equal, consumers obtain higher consumption utilities from having more friends. Second, there is an indirect effect, which is more subtle. When consumers become more connected, the competition between firms intensifies. This reduces firms' equilibrium prices and leads to more surplus to consumers.

On the firms' side, the preference over $\mathcal{G}_{n}$ actually varies with the parameters $\beta$ and $\delta$ as we have seen in Corollary 5 .

Theorem 3. Suppose Assumptions 1 and 2 hold. Then, there exists a positive number $\bar{\delta}>0$ such that, for any $\delta<\bar{\delta}$

1. if $\beta<\beta^{*} \approx 0.596$, the complete network is the optimal network structure for firms, i.e., $\mathbf{G}^{* \Pi}(\beta, \delta)=$ $\mathbf{K}_{n}$.

2. if $\beta>\beta^{*} \approx 0.596$, the empty network is the optimal network structure for firms, i.e., $\mathbf{G}^{* \Pi}(\beta, \delta)=$ $\mathbf{E}_{n}$.

Theorem 3 gives a clear-cut prediction on the optimal network structure for firms and shows that it critically depends on the level of product substitution $\beta$ between products and is independent of the consumers' intrinsic utility $(a)$ and the firms' marginal cost $(c)$. The intuition of this result is similar to that of the consumer surplus. A denser network is good for firms since it implies more consumption of both goods for the consumers. However, if the products are more substitutable (i.e. $\beta$ is higher), competition becomes fiercer. This reduces each firm's profitability when consumers become more connected and explains why the firms prefer the empty network when $\beta>\beta^{*}$. In contrast, where products are less substitutable (i.e. $\beta$ is lower), the firms benefit from higher consumption utilities since their products become more attractive and this makes the complete network desirable. In terms of total welfare, defined as the sum of $\mathbf{C S}^{*}$ and $\boldsymbol{\Pi}^{*}$, we deduce that, when $\beta<\beta^{*}, \mathbf{K}_{n}$ is the optimal network structure but, when $\beta>\beta^{*}$, the optimal network is undetermined.

In fact, the results of Theorems 2 and 3 could be used in order to analyze the equilibrium of a game where firms endogenously chose to allow consumers to form links or to subsidize the formation of social links. Indeed, consider a three-stage game where, in the first stage, consumers form links while the second (price determination) and third stage (consumption decisions) are as before. For network formation (first stage), consider the standard concept of pairwise stability

\footnotetext{
${ }^{27}$ For the complete network, Assumptions 1 reduces to $1-|\beta|-\delta(n-1)>0$.
} 
for equilibrium networks (Jackson and Wolinsky (1996)) and add a cost of forming a link. Then Theorems 2 and 3 show that, when the degree of substitution between the two products is not too high (i.e. $\beta<0.596$ ), then the optimal structure for both firms and consumers is the complete network. As a result, in that case, firms have as strong incentive to subsidize link formation (the consumer's cost of forming links) up to the point where the marginal cost of link equals the marginal gain in profit from having a denser network. On the contrary, if the degree of substitution between the two products is high enough (i.e. $\beta>0.596$ ), firms will impose a sufficiently high tax on link formation so that no consumer has an incentive to form a link since the empty network is the optimal structure for the firms.

Another way to understand the results of Theorems 2 and 3 is to assume that the network is given and that firms can decide whether or not they want to subsidize the weights $g_{i j}>0$ of the links between all consumers $i$ and $j .{ }^{28}$ Start with any network structure. Then, for small $\beta$, i.e. $\beta<0.596$, (the products are less substitutable), the firms always have incentives to subsidize $g_{i j}$ because they want consumers to interact more with each other since this leads to higher consumption. However, when $\beta$ becomes higher $(\beta>0.596)$, competition becomes fiercer and firms want consumers to interact less so that have no incentives to subsidize the weights of the links between consumers.

Uniform prices versus third-degree price discrimination. Suppose that there is a ban on third degree price discrimination so that all firms set the same price, regardless on consumers' position in the network. To see how this restriction affects the analysis, note that in a symmetric equilibrium with uniform prices, both firms choose $\mathbf{p}^{* A}=\mathbf{p}^{* B}=p^{u} \mathbf{1}_{n}$. Thus, the induced consumptions are $\mathbf{x}^{A}=\mathbf{x}^{B}=\mathbf{x}^{*}=\mathbf{M}^{+}\left(\mathbf{a}-p^{u} \mathbf{1}_{n}\right)$ by Corollary 1 , and, accordingly, the profits are $\Pi^{A}=\Pi^{B}=\left\langle\mathbf{x}^{*}, p^{u} \mathbf{1}_{n}-\mathbf{c}\right\rangle$. Since uniform pricing limits firms' best response to competitor's prices, hence affect the equilibrium prices and firm profits. The equilibrium under uniform pricing scheme is present in the following result.

Theorem 4. Suppose that Assumptions 1 and 2 hold and that both firms are restricted to charge uniform prices to all consumers. Then, there exists a unique equilibrium in the pricing stage where both firms charge $p^{u} \mathbf{1}_{n}$, where

$$
\begin{aligned}
p^{u} & =\frac{\left\langle\mathbf{1}_{n},\left(\mathbf{M}^{+} \mathbf{a}+\frac{\mathbf{M}^{+}+\mathbf{M}^{-}}{2} \mathbf{c}\right)\right\rangle}{\left\langle\mathbf{1}_{n},\left(\mathbf{M}^{+} \mathbf{1}_{n}+\frac{\mathbf{M}^{+}+\mathbf{M}^{-}}{2} \mathbf{1}_{n}\right)\right\rangle} \\
& =\frac{\left\langle\mathbf{1}_{n},\left[(1+\beta) \mathbf{I}_{n}-\delta \mathbf{G}\right]^{-1}(2 \mathbf{a}+\mathbf{c})\right\rangle+\left\langle\mathbf{1}_{n},\left[(1-\beta) \mathbf{I}_{n}-\delta \mathbf{G}\right]^{-1} \mathbf{c}\right\rangle}{\left\langle\mathbf{1}_{n}, 3\left[(1+\beta) \mathbf{I}_{n}-\delta \mathbf{G}\right]^{-1} \mathbf{1}_{n}\right\rangle+\left\langle\mathbf{1}_{n},\left[(1-\beta) \mathbf{I}_{n}-\delta \mathbf{G}\right]^{-1} \mathbf{1}_{n}\right\rangle} .
\end{aligned}
$$

The derivation of this theorem is similar to that of Theorem 1 . We observe that unless certain strong assumptions on network $\mathbf{G}$ are satisfied, the symmetric discriminatory price vectors derived in Theorem 1 and the uniform price vector derived in Theorem 4 are different. There-

\footnotetext{
${ }^{28}$ Our main results easily carry over to the case with a weighted network matrix where $g_{i j}$ takes nonnegative real values, not just $\{0,1\}$.
} 
fore, the competitive firms do exercise their price discrimination power at very refined levels. Interestingly, $p^{u}$ can be understood as an average over the prices that would prevail under price discrimination since, in the latter case, different consumers obtain different prices depending on their position in the network. ${ }^{29}$ More importantly, even if the firms are restricted to charge uniform prices, the matrix decomposition of demand functions (via $\mathbf{M}^{+}$and $\mathbf{M}^{-}$in (4)) still plays a crucial role in the market equilibrium. In other words, the network structure matters even though consumers are all charged the same price.

Let us now compare the welfare (both in terms of consumer surplus and firms' profits) between the uniform and the discrimination price model. For simplicity, and without loss of generality, we assume that $a_{i}=1, c_{i}=0$, for all $i$. Denote by

$$
\Delta \Pi \equiv \Pi^{u}-\Pi^{*} \text { and } \Delta C S \equiv C S^{u}-C S^{*}
$$

the difference in total profit and consumer surplus, respectively, between the uniform and the discrimination price model.

Proposition 8. Suppose that Assumptions 1 and 2 hold.

1. If $G$ is a regular network, or $\beta=0$, or $\delta=0$, then the total welfare (i.e. the sum of the equilibrium profits and consumer surplus) is the same under uniform and discriminatory pricing.

2. If $G$ is not a regular network and $\beta \neq 0$ and $\delta \neq 0$, then, for low enough $\delta$, there exist two positive thresholds $\widehat{\beta}(\delta)$ and $\widetilde{\beta}(\delta)$ such that

$$
\Delta \Pi \gtreqless 0 \Longleftrightarrow \beta \lesseqgtr \widehat{\beta}(\delta)
$$

and

$$
\Delta C S \gtreqless 0 \Longleftrightarrow \beta \gtreqless \widetilde{\beta}(\delta) .
$$

This interesting result shows that, when the network is not regular, and network effects are small (low $\delta$ ), then $\beta$ has to be small enough for the firms' profit to be higher under uniform pricing while $\beta$ has to be large enough for the consumer surplus to be higher under uniform pricing. To interpret this result, we first note that in equilibrium consumer surplus can be expressed as $(1+\beta)$ times the sum of the square terms of their equilibrium consumptions (see (15)). Therefore, the more disperse the consumptions are, the higher consumer surplus is generated. Under uniform pricing, the firms are restricted to offer the same prices for all consumers, and more central players tend to consume more. By contrast, in the discriminatory pricing regime the firms can charge charge a lower price to more central players; this further enlarges the consumption discrepancy between more and less central players. Thus, uniform pricing tends to reduce the consumption variation and hurts consumer surplus. This, however, is only half of the story.

\footnotetext{
${ }^{29}$ Observe in Figure 5, the uniform price curve lies between the prices for the center (below) and the periphery nodes (above).
} 
Uniform pricing also restricts firms' ability to conduct discrimination and extract revenue from heterogeneous consumers.

The above two conflicting forces collectively give rise to the findings in Proposition 8. Recall that the higher is $\beta$, the more the products are substitutable (i.e. the less differentiated they are), and, thus, the higher is the competition between the two firms. As a result, an increase of $\beta$ leads to a lower price on average, and enlarges the consumption variation among more and less central players. When $\beta$ is low, there is less competition to attract consumers. Since the network effects are small (i.e. small $\delta$ ), firms prefers uniform pricing because what they lose on the central consumers is not enough compensated by what they gain on the less central consumers. Obviously, this is the reverse for the consumers who prefer the discriminatory pricing regime when $\beta$ is small. On the contrary, when $\beta$ is high enough, competition becomes fiercer and firms obtain higher profits under discriminatory pricing policy.

We hereby provide some simple numerical simulations that confirm this intuition even when $\delta$ has higher values. Consider the star network with one center and two periphery nodes. In Figure 3, we study an increase in $\beta$ on $\Delta \Pi$ (left panel) and on $\Delta C S$ (right panel) for $\delta=0.1$ (dashed curve) and $\delta=0.2$ (solid curve). Even when $\delta>0$, we still obtain results similar to that of Proposition 8. Indeed, we see that, for $\Delta \Pi$ (left panel), the sign is positive (i.e. $\Pi^{u}>\Pi^{*}$ ) for small $\beta$ and negative (i.e. $\Pi^{u}<\Pi^{*}$ ) for large $\beta$. Moreover, the cutoff value of $\beta$ is decreasing in $\delta$. For $\triangle C S$, we obtain the reverse results as stated in Proposition 8.
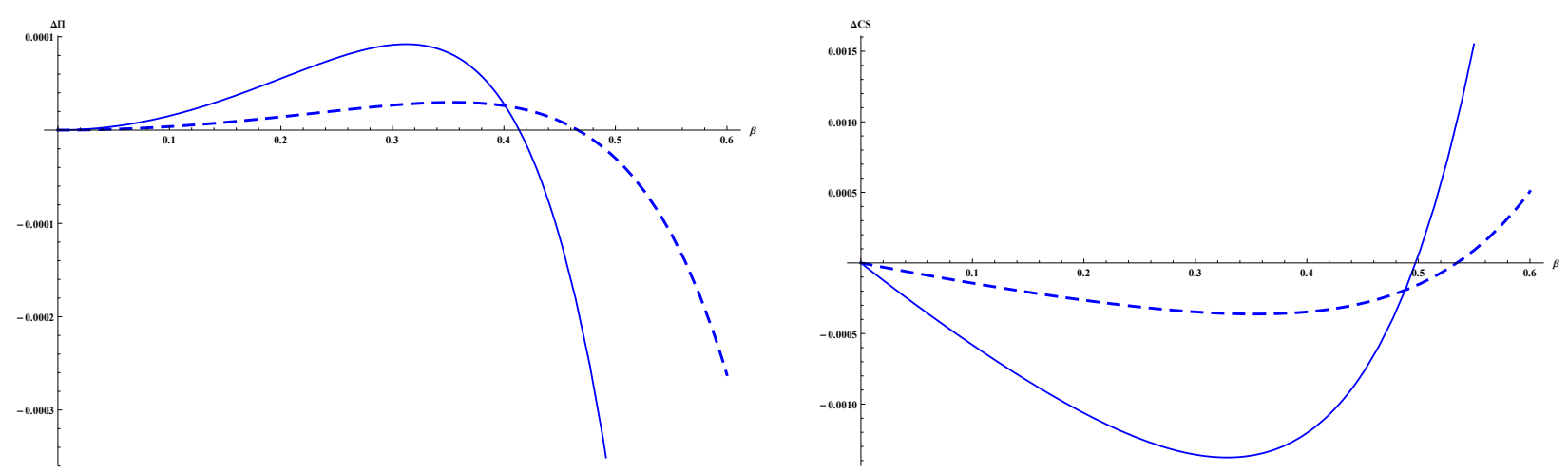

Figure 3: $\mathrm{x}$-axis is $\beta$; y-axis, left figure is $\Delta \Pi$, while right figure is $\Delta C S$; dashed line is for $\delta=0.1$, solid line is for $\delta=0.2$.

In Figure 4 , in the plan $(\beta, \delta)$, we plot the regions where $\Delta \Pi$ is positive (darker area) or negative (lighter area) in the left panel and the regions where $\Delta C S$ is positive (darker area) or negative (lighter area) in the right panel. We see that, when $\beta$ is small, the uniform price is preferred (i.e. $\Pi^{u}>\Pi^{*}$ ), even when $\delta$ is relatively high. However, when $\beta$ is large enough (above 0.5 ) so that competition becomes very fierce, then firms are better off under discriminatory pricing even when $\delta$ is close to zero. For the consumer surplus (right panel of Figure 4), the pattern is reversed.

Finally, in Figure 5, in the plane ( $\beta$, price), we plot three curves where the uniform price curve lies between the prices for the center (below) and the periphery nodes (above). The left 

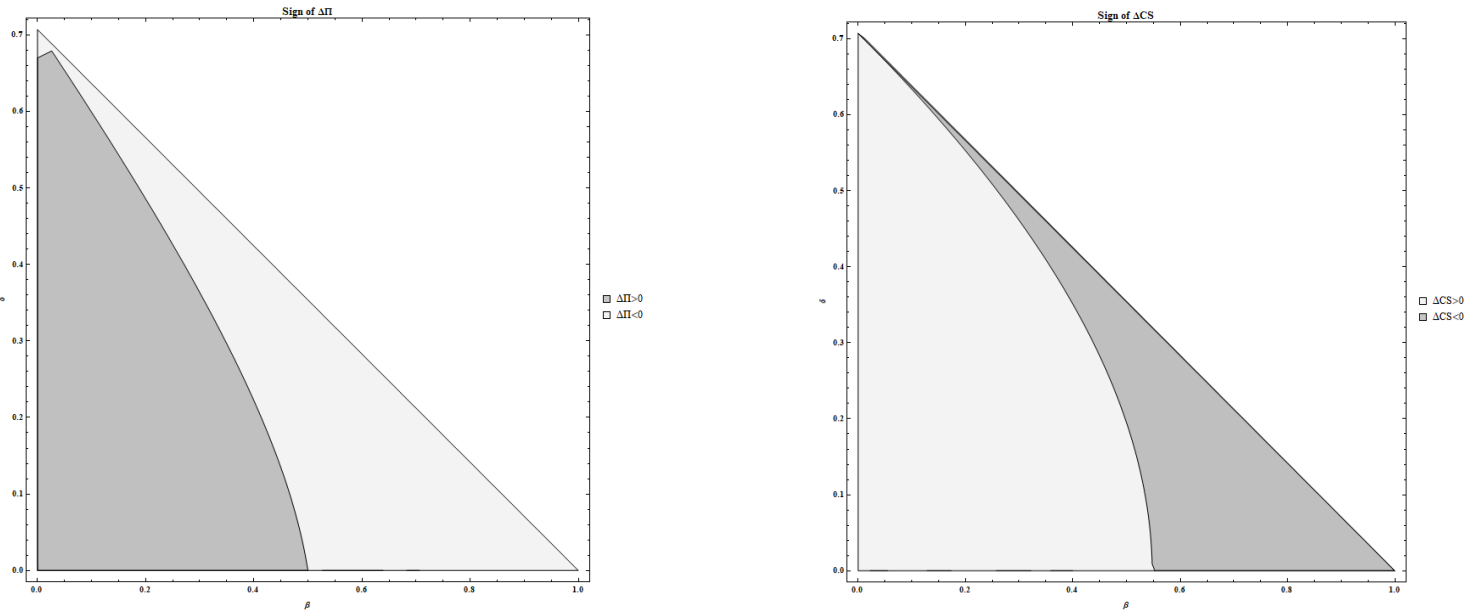

Figure 4: $\mathrm{x}$-axis is $\beta ; \mathrm{y}$-axis is $\delta$. Sign of $\Delta \Pi$ (left panel) and $\Delta C S$ (right panel), the sign is positive on the dark area, negative on the light area.

panel considers a $\delta=0.1$ while, in the right panel, $\delta=0.2$. We see that the difference in prices between the different schemes increases for larger $\beta$ or larger $\delta$. In particular, when $\beta$ increases, so that competition becomes fiercer, there are larger differences between the price charged to the periphery consumers and the one charged to the central consumer. These differences are even bigger when $\delta$ is higher because the central agent generates even more externalities to the two periphery consumers and thus deserves a higher discount.
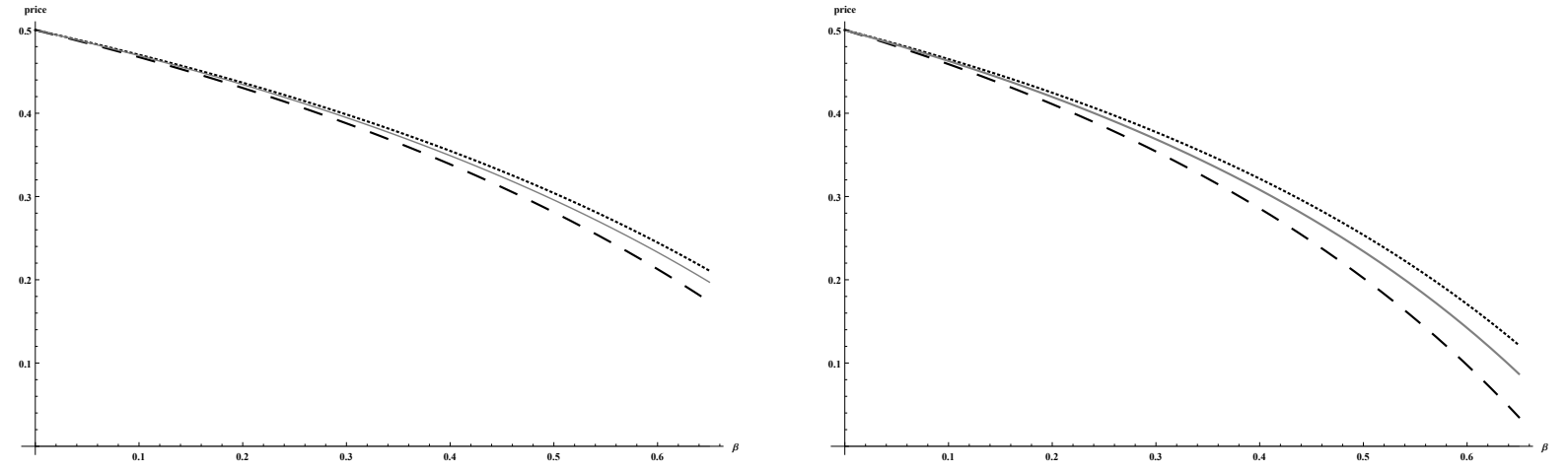

Figure 5: $\mathrm{x}$-axis is $\beta$; $\mathrm{y}$-axis is price. Left figure: $\delta=0.1$, right figure $\delta=0.2$; Dotted line: price of periphery node; Dashed line: price of center node; Solid line: uniform price.

\section{Conclusion}

In this paper, we consider a duopoly setting in which two firms sell two interdependent products to the consumers in a social network. Consumers are endowed with heterogeneous intrinsic valuations for the products and firms bear different production costs. Moreover, there are network externalities (strategic complementarities) amongst the consumers in terms of their consumption 
utilities. We provide a full characterization of the equilibrium prices and provide conditions under which the subgame perfect Nash equilibrium exists and is unique. Contrary to the monopoly case, we show that the equilibrium prices exhibit strong network dependence, which implies that the knowledge of the network structure is crucial for profit maximization. We provide some examples of networks that illustrate how the competitive prices depend on the consumers' relative positions. We also show that when the firms sell substitutable products, enhancing network externalities among consumers pushes the equilibrium price downwards. Moreover, we show that firms' equilibrium profits can be reduced when either the network becomes denser or the network effect is strengthened. Therefore, competition can lead to important distinct implications of the pricing strategies as well as of the firms' profitability.

We also investigate the welfare implications of our model. We find that consumers always benefit from being more connected to each other because this increases their consumption utilities and, at the same time, intensifies firms' competition. This suggests that the complete network maximizes consumer welfare. In contrast, firms may either prefer the complete network or the empty network, depending on the degree of product substitution. Finally, we compare uniform pricing and discriminatory pricing. We show that when the network is not regular, firms obtain higher profits under uniform pricing when the products are sufficiently differentiated. In contrast, consumers are better off under uniform pricing than under discriminatory pricing when the products are highly substitutable.

In this paper, we adopt the linear quadratic payoffs to deliver clean and intuitive results. As demonstrated by Bramoullé et al. (2014), the crucial assumption to facilitate such analysis is that players' best responses are linear. Therefore, the payoff functions can be generalized following their approach. However, going beyond the family of linear best responses, only qualitative results may be more likely to survive. For example, we anticipate that the network structure still affects the equilibrium outcomes, though we do not expect to obtain the clean characterization shown in Theorem 1. Furthermore, it is unlikely to establish linear relationships between equilibrium prices and centrality of players, even though we conjecture that more central players are likely to receive larger discounts. For the welfare analysis on the network structure, we expect that consumers would still prefer denser networks due to lower prices and larger network externalities. For firms, they will still face the fundamental trade-off between the negative effect through intensified price competition and the positive demand enhancing effect through larger network effects. That being said, thorough investigations are required to advance our knowledge towards more general payoff functions.

In our setup, the firms are restricted to set per unit charges. Allowing for two-part tariffs significantly change the strategy space of the firms. In general, the results can be quite different because both firms know that the players are located in heterogeneous network positions. Thus, even if the firms are not allowed to set discriminatory prices for different players, with two-part tariffs, second-degree discrimination is inevitable. Second-degree price discrimination occurs here because players in different network positions derive different consumption utilities from the same consumption level. The nonlinear pricing literature has already identified the 
substantial difficulties when competing firms (sellers) attempt to price discriminate players that have heterogeneous willingness to pay in a vertically differentiated setup (e.g., Armstrong and Vickers (2010)). We expect that the analysis will be more involved here because the network structure is explicitly taken into account. This deserves a separate study that we leave for future research.

\section{References}

Ambrus, A. and R. Argenziano (2009). Asymmetric networks in two-sided markets. American Economic Journal: Microeconomics 1(1), 17-52.

Aoyagi, M. (2014). Bertrand competition under network externalities. Working paper, Osaka University.

Armstrong, M. (2006). Competition in two-sided markets. The RAND Journal of Economics 37(3), 668-691.

Armstrong, M. and J. Vickers (2010). Competitive non-linear pricing and bundling. The Review of Economic Studies 77(1), 30-60.

Arthur, D., R. Motwani, A. Sharma, and Y. Xu (2009). Pricing strategies for viral marketing on social networks. In S. Leonardi (Ed.), Internet and Network Economics, pp. 101-112.

Ballester, C., A. Calvó-Armengol, and Y. Zenou (2006). Who's who in networks. wanted: the key player. Econometrica 74(5), 1403-1417.

Banerji, A. and B. Dutta (2009). Local network externalities and market segmentation. International Journal of Industrial Organization 27(5), 605-614.

Belhaj, M., S. Bervoets, and F. Deroïan (2016). Efficient networks in games with local complementarities. Theoretical Economics 11(1), 357-380.

Belhaj, M. and F. Deroïan (2016). The value of network information: Assortative mixing makes the difference. Unpublished manuscript, GREQAM.

Billand, P., B. C., S. Chakrabarti, and S. Sarangi (2014). A note on networks of collaboration in multi-market oligopolies. Annals of Regional Science 53, 325-335.

Bimpikis, K., S. Ehsani, and R. Ilkilic (2015). Cournot competition in networked markets. Unpublished paper, Stanford University.

Bimpikis, K., A. Ozdaglar, and E. Yildiz (2016). Competitive targeted advertising over networks. Operations Research 64(3), 705-720.

Bloch, F. (2016). Targeting and pricing in social networks. In: Y. Bramoullé, A. Galeotti and B. Rogers (Eds.), Oxford Handbook of the Economics of Networks, Oxford: Oxford University Press. 
Bloch, F. and N. Quérou (2013). Pricing in social networks. Games and Economic Behavior 80, 243-261.

Bonacich, P. (1987). Power and centrality: A family of measures. American journal of sociology 92(5), $1170-1182$.

Bramoullé, Y., R. Kranton, and M. d'Amours (2014). Strategic interaction and networks. American Economic Review 104, 898-930.

Bramoullé, Y., R. Kranton, and M. D'amours (2014). Strategic interaction and networks. The American Economic Review 104(3), 898-930.

Cabral, L., D. J. Salant, and G. A. Woroch (1999). Monopoly pricing with network externalities. International Journal of Industrial Organization 17(2), 199-214.

Caillaud, B. and B. Jullien (2003). Chicken \& egg: Competition among intermediation service providers. RAND Journal of Economics 34(2), 309-328.

Candogan, O., K. Bimpikis, and A. Ozdaglar (2012). Optimal pricing in networks with externalities. Operations Research 6o(4), 883-905.

Carroni, E. and S. Righi (2015). Pricing in social networks under limited information. University of Namur, Department of Economics Working Papers No. 2015/03.

Chen, Y.-J., Y. Zenou, and J. Zhou (2017). Multiple activities in networks. American Economic Journal: Microeconomics, forthcoming.

Combes, P.-P., T. Mayer, and J.-F. Thisse (2008). Economic Geography: The Integration of Regions and Nations. Princeton: Princeton University Press.

Currarini, S. and F. Feri (2015). Information sharing networks in linear quadratic games. International Journal of Game Theory 44, 701-732.

Deroïan, F. and F. Gannon (2006). Quality-improving alliances in differentiated oligopoly. International Journal of Industrial Organization 24, 629-637.

Dixit, A. (1979). A model of duopoly suggesting a theory of entry barriers. Bell Journal of Economics $10(1), 20-32$.

Dybvig, P. H. and C. S. Spatt (1983). Adoption externalities as public goods. Journal of Public Economics 20(2), 231-247.

Economides, N. (1996). The economics of networks. International Journal of Industrial Organization $14,673-699$.

Fainmesser, I. and A. Galeotti (2016). Pricing network effects. Review of Economic Studies 83(1), $165-198$. 
Fainmesser, I. P. and A. Galeotti (2017). Pricing network effects: Competition. working paper, available at SSRN: https://ssrn.com/abstract $=\mathbf{2 8 7 4 8 8 6 .}$

Farrell, J. and G. Saloner (1986). Installed base and compatibility: Innovation, product preannouncement, and predation. American Economic Review 76, 940-955.

Fazeli, A. and A. Jadbabaie (2012). Duopoly pricing game in networks with local coordination effects. In Decision and Control (CDC), 2012 IEEE 51st Annual Conference on, pp. 2684-2689. IEEE.

Grabisch, M., A. Mandel, A. Rusinowska, and E. Tanimura (2018). Strategic influence in social networks. Mathematics of Operations Research 43(1), 29-50.

Hartline, J., V. Mirrokni, and M. Sundararajan (2008). Optimal marketing strategies over social networks. In Proceedings of the 17th international conference on World Wide Web, pp. 189-198. ACM.

Hiller, T. (2017). Peer effects in endogenous networks. Games and Economic Behavior 105, 349-367.

Ioannides, Y. (2012). From Neighborhoods to Nations: The Economics of Social Interactions. Princeton: Princeton University Press.

Jackson, M. (2008). Social and Economic Networks. Princeton: Princeton University Press.

Jackson, M. and A. Wolinsky (1996). A strategic model of social and economic networks. Journal of Economic Theory 71, 44-74.

Jackson, M. and Y. Zenou (2015). Games on networks. In P. Young and S. Zamir (Eds.), Handbook of Game Theory, Vol. 4, pp. 34-61. Amsterdam: Elsevier Publisher.

Jackson, M. O., B. W. Rogers, and Y. Zenou (2017). The economic consequences of social-network structure. Journal of Economic Literature 55(1), 49-95.

Jullien, B. (2011). Competition in multi-sided markets: Divide and conquer. American Economic Journal: Microeconomics 3(4), 186-220.

Katz, L. (1953). A new status index derived from sociometric analysis. Psychometrika 18(1), 39-43.

Katz, M. and C. Shapiro (1985). Network externalities, competition, and compatibility. The American Economic Review 75(3), 424-440.

König, M. D., X. Liu, and Y. Zenou (2016). R\&d networks: Theory, empirics and policy implications. Unpublished manuscript, Monash University.

Leduc, M. V., M. O. Jackson, and R. Johari (2017). Pricing and referrals in diffusion on networks. Games and Economic Behavior 104, 568-594.

Lobel, I., E. Sadler, and L. R. Varshney (2016). Customer referral incentives and social media. Management Science 63(10), 3514-3529. 
Melitz, M. (2003). The impact of trade on intra-industry reallocations and aggregate industry productivity. Econometrica $71,1695-1725$.

Ochs, J. and I.-U. Park (2010). Overcoming the coordination problem: Dynamic formation of networks. Journal of Economic Theory 145(2), 689-720.

Ottaviano, G., T. Tabuchi, and J.-F. Thisse (2002). Agglomeration and trade revisited. International Economic Review 43, 409-436.

Rao, A., N. Spasojevic, Z. Li, and T. DSouza (2015). Klout score: Measuring influence across multiple social networks. In Big Data (Big Data), 2015 IEEE International Conference on, pp. 2282-2289. IEEE.

Rochet, J.-C. and J. Tirole (2006). Two-sided markets: A progress report. The RAND Journal of Economics 37(3), 645-667.

Rohlfs, J. (1974). A theory of interdependent demand for a communications service. The Bell Journal of Economics and Management Science 5(1), 16-37.

Shi, M. (2013). Social network-based discriminatory pricing strategy. Marketing Letters 14, 239256.

Shin, E. (2017). Monopoly pricing and diffusion of social network goods. Games and Economic Behavior 102, 162-178.

Singh, N. and X. Vives (1984). Price and quantity competition in a differentiated duopoly. RAND Journal of Economics 15(4), 546-554.

Ushchev, P. and Y. Zenou (2015). Price competition in product variety networks. CEPR Discussion Paper No. 10862.

Zhang, Y. and Y.-J. Chen (2018). Optimal nonlinear pricing in social networks under asymmetric network information. Unpublished manuscript, Hong Kong University of Science and Technology.

Zhou, J. and Y.-J. Chen (2015). Key leaders in social networks. Journal of Economic Theory 157, 212-235.

Zhou, J. and Y.-J. Chen (2017). Optimal pricing with sequential consumption in networks. Operations Research, forthcoming. 


\section{Appendix: Proofs for the results in the main text}

Proof of Proposition 1: The first-order conditions are given by:

$$
\left[\begin{array}{ll}
1 & \beta \\
\beta & 1
\end{array}\right]\left[\begin{array}{c}
x_{i}^{A} \\
x_{i}^{B}
\end{array}\right]=\left[\begin{array}{l}
a_{i}^{A}-p_{i}^{A}+\delta \sum_{j=1}^{n} g_{i j} x_{j}^{A} \\
a_{i}^{B}-p_{i}^{B}+\delta \sum_{j=1}^{n} g_{i j} x_{j}^{B}
\end{array}\right] .
$$

Taking the sum of first-order conditions in (16) yields:

$$
(1+\beta) \frac{\left(x_{i}^{A}+x_{i}^{B}\right)}{2}=\frac{\left(a_{i}^{A}+a_{i}^{B}\right)}{2}-\frac{\left(p_{i}^{A}+p_{i}^{B}\right)}{2}+\delta \sum_{j=1}^{n} g_{i j} \frac{\left(x_{j}^{A}+x_{j}^{B}\right)}{2},
$$

which can be expressed in matrix form:

$$
(1+\beta) \frac{\left(\mathbf{x}^{A}+\mathbf{x}^{B}\right)}{2}=\frac{\left(\mathbf{a}^{A}+\mathbf{a}^{B}\right)}{2}-\frac{\left(\mathbf{p}^{A}+\mathbf{p}^{B}\right)}{2}+\delta \mathbf{G} \frac{\left(\mathbf{x}^{A}+\mathbf{x}^{B}\right)}{2} .
$$

Therefore, we obtain

$$
\frac{\left(\mathbf{x}^{A}+\mathbf{x}^{B}\right)}{2}=\left[(1+\beta) \mathbf{I}_{n}-\delta \mathbf{G}\right]^{-1}\left[\left(\frac{\mathbf{a}^{A}+\mathbf{a}^{B}}{2}\right)-\left(\frac{\mathbf{p}^{A}+\mathbf{p}^{B}}{2}\right)\right] .
$$

On the other hand, taking the difference in (16) yields:

$$
\begin{aligned}
&(1-\beta) \frac{\left(x_{i}^{A}-x_{i}^{B}\right)}{2}=\frac{\left(a_{i}^{A}-a_{i}^{B}\right)}{2}-\frac{\left(p_{i}^{A}-p_{i}^{B}\right)}{2}+\delta \sum_{j=1}^{n} g_{i j} \frac{\left(x_{j}^{A}-x_{j}^{B}\right)}{2} \\
& \Longrightarrow \quad(1-\beta) \frac{\left(\mathbf{x}^{A}-\mathbf{x}^{B}\right)}{2}=\frac{\left(\mathbf{a}^{A}-\mathbf{a}^{B}\right)}{2}-\frac{\left(\mathbf{p}^{A}-\mathbf{p}^{B}\right)}{2}+\delta \mathbf{G} \frac{\left(\mathbf{x}^{A}-\mathbf{x}^{B}\right)}{2} .
\end{aligned}
$$

Therefore,

$$
\frac{\left(\mathbf{x}^{A}-\mathbf{x}^{B}\right)}{2}=\left[(1-\beta) \mathbf{I}_{n}-\delta \mathbf{G}\right]^{-1}\left[\left(\frac{\mathbf{a}^{A}-\mathbf{a}^{B}}{2}\right)-\left(\frac{\mathbf{p}^{A}-\mathbf{p}^{B}}{2}\right)\right] .
$$

Combing equations (17) and (18), we obtain (4). Finally, Assumption 1 guarantees that the two matrices: $\left[(1+\beta) \mathbf{I}_{n}-\delta \mathbf{G}\right]$ and $\left[(1-\beta) \mathbf{I}_{n}-\delta \mathbf{G}\right]$ are positive definite, hence invertible (i.e. nonsingular).

Proof of Corollary $\mathbf{1}$ : When $\mathbf{a}^{A}=\mathbf{a}^{B}=\mathbf{a}$ and $\mathbf{p}^{A}=\mathbf{p}^{B}=\mathbf{p}$, by (4) we have:

$$
\begin{aligned}
\mathbf{x}^{A} & =\mathbf{M}^{+}\left\{\frac{\mathbf{a}^{A}+\mathbf{a}^{B}}{2}-\frac{\mathbf{p}^{A}+\mathbf{p}^{B}}{2}\right\}+\mathbf{M}^{-} \underbrace{\left\{\frac{\mathbf{a}^{A}-\mathbf{a}^{B}}{2}-\frac{\mathbf{p}^{A}-\mathbf{p}^{B}}{2}\right\}}_{=\mathbf{0} \text { as } \mathbf{a}^{A}=\mathbf{a}^{B}, \text { and } \mathbf{p}^{A}=\mathbf{p}^{B}} \\
& =\mathbf{M}^{+}(\mathbf{a}-\mathbf{p}) .
\end{aligned}
$$

By the same logic, $\mathbf{x}^{B}=\mathbf{M}^{+}(\mathbf{a}-\mathbf{p})$. 
Proof of Theorem 1: First, we derive the best-reply function from each firm's perspective.

Best response pricing. Suppose the price of the second firm $\mathbf{p}^{B}$ is fixed and we are interested in firm $A^{\prime}$ s best response. We have seen that the profit of each firm is given by: $\Pi^{A}(\mathbf{p})=$ $\left\langle\mathbf{p}^{A}-\mathbf{c}^{A}, \mathbf{x}^{A}\left(\mathbf{p}^{A}, \mathbf{p}^{B}\right)\right\rangle$ and $\Pi^{B}(\mathbf{p})=\left\langle\mathbf{p}^{B}-\mathbf{c}^{B}, \mathbf{x}^{B}\left(\mathbf{p}^{A}, \mathbf{p}^{B}\right)\right\rangle$. Then, by using the demand functions $\mathbf{x}^{A}$ and $\mathbf{x}^{B}$ given in Proposition 1 , the profit of firms $A$ and $B$ can be expressed as:

$$
\mathbf{\Pi}^{A}(\mathbf{p})=\left\langle\mathbf{p}^{A}-\mathbf{c}^{A},\left(\frac{\mathbf{M}^{+}+\mathbf{M}^{-}}{2}\right)\left(\mathbf{a}^{A}-\mathbf{p}^{A}\right)+\left(\frac{\mathbf{M}^{+}-\mathbf{M}^{-}}{2}\right)\left(\mathbf{a}^{B}-\mathbf{p}^{B}\right)\right\rangle
$$

and

$$
\boldsymbol{\Pi}^{B}(\mathbf{p})=\left\langle\mathbf{p}^{A}-\mathbf{c}^{A},\left(\frac{\mathbf{M}^{+}-\mathbf{M}^{-}}{2}\right)\left(\mathbf{a}^{A}-\mathbf{p}^{A}\right)+\left(\frac{\mathbf{M}^{+}+\mathbf{M}^{-}}{2}\right)\left(\mathbf{a}^{B}-\mathbf{p}^{B}\right)\right\rangle
$$

The first-order condition is given by:

$$
\left(\frac{\mathbf{M}^{+}+\mathbf{M}^{-}}{2}\right)\left(\mathbf{a}^{A}-\mathbf{p}^{A}\right)+\left(\frac{\mathbf{M}^{+}-\mathbf{M}^{-}}{2}\right)\left(\mathbf{a}^{B}-\mathbf{p}^{B}\right)-\left(\frac{\mathbf{M}^{+}+\mathbf{M}^{-}}{2}\right)\left(\mathbf{p}^{A}-\mathbf{c}^{A}\right)=\mathbf{0}
$$

or equivalently

$$
\left(\mathbf{M}^{+}+\mathbf{M}^{-}\right)\left(\mathbf{a}^{A}+\mathbf{c}^{A}-2 \mathbf{p}^{A}\right)=-\left(\mathbf{M}^{+}-\mathbf{M}^{-}\right)\left(\mathbf{a}^{B}-\mathbf{p}^{B}\right) .
$$

Therefore, the best response for firm $A$ is equal to:

$$
\mathbf{B R}^{A}\left(\mathbf{p}^{B}\right)=\left(\frac{\mathbf{a}^{A}+\mathbf{c}^{A}}{2}\right)+\frac{1}{2}\left(\mathbf{M}^{+}+\mathbf{M}^{-}\right)^{-1}\left(\mathbf{M}^{+}-\mathbf{M}^{-}\right)\left(\mathbf{a}^{B}-\mathbf{p}^{B}\right)
$$

In a similar way, we can obtain the best response for firm $B$. We obtain:

$$
\mathbf{B} \mathbf{R}^{B}\left(\mathbf{p}^{A}\right)=\left(\frac{\mathbf{a}^{B}+\mathbf{c}^{B}}{2}\right)+\frac{1}{2}\left(\mathbf{M}^{+}+\mathbf{M}^{-}\right)^{-1}\left(\mathbf{M}^{+}-\mathbf{M}^{-}\right)\left(\mathbf{a}^{B}-\mathbf{p}^{B}\right)
$$

It is easily verified that both best-reply functions are monotone, i.e. $\mathbf{B} \mathbf{R}^{B}\left(\mathbf{p}^{A}\right)$ is increasing in $\mathbf{p}^{A}$ and $\mathbf{B} \mathbf{R}^{A}\left(\mathbf{p}^{B}\right)$ is increasing in $\mathbf{p}^{B}$.

Second order condition. From (19), we note that firm $A^{\prime}$ 's profit function is strictly concave in $\mathbf{p}^{A}$, as the Hessian matrix $\frac{\partial^{2} \Pi^{A}}{\partial\left(\mathbf{p}^{A}\right)^{2}}=-\left(\mathbf{M}^{+}+\mathbf{M}^{-}\right) / 2$ is negative definite as both matrices $\mathbf{M}^{+}$ and $\mathbf{M}^{-}$are positive definite by Assumption 1. So the second-order condition indeed holds.

Equilibrium prices. To determine the equilibrium prices, we need to solve these two bestreply functions simultaneously, that is:

$$
\left\{\begin{array}{l}
\mathbf{p}^{* A}=\mathbf{B} \mathbf{R}^{A}\left(\mathbf{p}^{* B}\right)=\left(\frac{\mathbf{a}^{A}+\mathbf{c}^{A}}{2}\right)+\frac{1}{2}\left(\mathbf{M}^{+}+\mathbf{M}^{-}\right)^{-1}\left(\mathbf{M}^{+}-\mathbf{M}^{-}\right)\left(\mathbf{a}^{B}-\mathbf{p}^{B}\right) \\
\mathbf{p}^{* B}=\mathbf{B} \mathbf{R}^{B}\left(\mathbf{p}^{* A}\right)=\left(\frac{\mathbf{a}^{B}+\mathbf{c}^{B}}{2}\right)+\frac{1}{2}\left(\mathbf{M}^{+}+\mathbf{M}^{-}\right)^{-1}\left(\mathbf{M}^{+}-\mathbf{M}^{-}\right)\left(\mathbf{a}^{B}-\mathbf{p}^{B}\right)
\end{array}\right.
$$

Using the semi-symmetry (SS) assumption given by Assumption 2, i.e. $\mathbf{a}^{A}=\mathbf{a}^{B}=\mathbf{a}$ and 
$\mathbf{c}^{A}=\mathbf{c}^{B}=\mathbf{c}$, we easily obtain the symmetric equilibrium $\mathbf{p}^{* A}=\mathbf{p}^{* B}=\mathbf{p}^{*}$, given by:

$$
\begin{gathered}
\mathbf{p}^{*}=\left(\frac{\mathbf{a}+\mathbf{c}}{2}\right)+\frac{1}{2}\left(\mathbf{M}^{+}+\mathbf{M}^{-}\right)^{-1}\left(\mathbf{M}^{+}-\mathbf{M}^{-}\right)\left(\mathbf{a}-\mathbf{p}^{*}\right) \\
\Leftrightarrow 2 \mathbf{p}^{*}=\mathbf{a}+\mathbf{c}+\left(\mathbf{M}^{+}+\mathbf{M}^{-}\right)^{-1}\left(\mathbf{M}^{+}-\mathbf{M}^{-}\right)\left(\mathbf{a}-\mathbf{p}^{*}\right) \\
\Leftrightarrow\left(\mathbf{p}^{*}-\mathbf{c}\right)=\left(\mathbf{a}-\mathbf{p}^{*}\right)+\left(\mathbf{M}^{+}+\mathbf{M}^{-}\right)^{-1}\left(\mathbf{M}^{+}-\mathbf{M}^{-}\right)\left(\mathbf{a}-\mathbf{p}^{*}\right) \\
\Leftrightarrow\left(\mathbf{M}^{+}+\mathbf{M}^{-}\right)\left(\mathbf{p}^{*}-\mathbf{c}\right)=\left(\mathbf{M}^{+}+\mathbf{M}^{-}\right)\left(\mathbf{a}-\mathbf{p}^{*}\right)+\left(\mathbf{M}^{+}-\mathbf{M}^{-}\right)\left(\mathbf{a}-\mathbf{p}^{*}\right) \\
\Leftrightarrow\left(\mathbf{M}^{+}+\mathbf{M}^{-}\right)\left(\mathbf{p}^{*}-\mathbf{c}\right)=2 \mathbf{M}^{+}\left(\mathbf{a}-\mathbf{p}^{*}\right) \\
\Leftrightarrow \mathbf{M}^{+}\left(\mathbf{a}-\mathbf{p}^{*}\right)=\frac{1}{2}\left(\mathbf{M}^{+}+\mathbf{M}^{-}\right)\left(\mathbf{p}^{*}-\mathbf{c}\right)
\end{gathered}
$$

which is equation (9). Moreover, we have:

$$
\begin{gathered}
\mathbf{M}^{+}\left(\mathbf{a}-\mathbf{p}^{*}\right)=\frac{1}{2}\left(\mathbf{M}^{+}+\mathbf{M}^{-}\right)\left(\mathbf{p}^{*}-\mathbf{c}\right) \\
\Leftrightarrow 2 \mathbf{M}^{+} \mathbf{a}-\mathbf{2} \mathbf{M}^{+} \mathbf{p}^{*}=\left(\mathbf{M}^{+}+\mathbf{M}^{-}\right) \mathbf{p}^{*}-\left(\mathbf{M}^{+}+\mathbf{M}^{-}\right) \mathbf{c} \\
\Leftrightarrow \mathbf{p}^{*}=\left(3 \mathbf{M}^{+}+\mathbf{M}^{-}\right)^{-1}\left[2 \mathbf{M}^{+} \mathbf{a}+\left(\mathbf{M}^{+}+\mathbf{M}^{-}\right) \mathbf{c}\right]
\end{gathered}
$$

We then have:

$$
\begin{aligned}
\mathbf{p}^{*} & =\left(3 \mathbf{M}^{+}+\mathbf{M}^{-}\right)^{-1}\left[2 \mathbf{M}^{+} \mathbf{a}+\left(\mathbf{M}^{+}+\mathbf{M}^{-}\right) \mathbf{c}\right] \\
& =\left[(2-\beta) \mathbf{I}_{n}-2 \delta \mathbf{G}\right]^{-1}\left\{\left((1-\beta) \mathbf{I}_{n}-\delta \mathbf{G}\right) \mathbf{a}+\left(\mathbf{I}_{n}-\delta \mathbf{G}\right) \mathbf{c}\right\} \\
& =\mathbf{c}+\left[(2-\beta) \mathbf{I}_{n}-2 \delta \mathbf{G}\right]^{-1}\left[(1-\beta) \mathbf{I}_{n}-\delta \mathbf{G}\right](\mathbf{a}-\mathbf{c}) \\
& =\left(\frac{\mathbf{a}+\mathbf{c}}{2}\right)-\frac{\beta}{2}\left[(2-\beta) \mathbf{I}_{n}-2 \delta \mathbf{G}\right]^{-1}(\mathbf{a}-\mathbf{c}) .
\end{aligned}
$$

which is equation (7). Last, this equilibrium is unique because Theorem 1 is just a special case of Theorem C.2, which is stated in Appendix C.4 and where the proof of Theorem C.2 is given in Appendix F.

Proof of Corollary 2: Recall that, by (7)

$$
\mathbf{p}^{*}=\frac{\mathbf{a}+\mathbf{c}}{2}-\frac{\beta}{2}\left[(2-\beta) \mathbf{I}_{n}-2 \delta \mathbf{G}\right]^{-1}(\mathbf{a}-\mathbf{c}) .
$$

When $\delta=0$ (no network effect),

$$
\mathbf{p}^{*}=\frac{\mathbf{a}+\mathbf{c}}{2}-\frac{\beta}{2(2-\beta)}(\mathbf{a}-\mathbf{c})=\frac{(1-\beta) \mathbf{a}+\mathbf{c}}{2-\beta} .
$$

When $\beta=0$ (two independent networks),

$$
\mathbf{p}^{*}=\frac{\mathbf{a}+\mathbf{c}}{2},
$$


which clearly is independent of parameter $\delta$ and network $\mathbf{G}$.

For the second part, first observe that

$$
\left.\frac{\partial \mathbf{p}^{*}}{\partial \delta}\right|_{\delta=0}=-\frac{\beta}{(2-\beta)^{2}} \mathbf{G}(\mathbf{a}-\mathbf{c}) .
$$

If $\mathbf{p}^{*}$ is independent of $\delta,-\frac{\beta}{(2-\beta)^{2}} \mathbf{G}(\mathbf{a}-\mathbf{c})=\mathbf{0}$. This holds only if $\beta=0$, as $\mathbf{G}(\mathbf{a}-\mathbf{c})$ is a nonzero vector. Notice that if $\mathbf{G}$ is an empty network, $\mathbf{p}^{*}$ obviously does not depend on $\delta$.

For the third part,

$$
\frac{\partial p_{i}}{\partial a_{j}}=-\frac{\beta}{2(2-\beta)} m_{i j}\left(\mathbf{G}, \frac{2 \delta}{2-\beta}\right)
$$

Since $g_{i j}>0, \delta>0$, we have $m_{i j}\left(\mathbf{G}, \frac{2 \delta}{2-\beta}\right)>0$ as well. As a result, if $p_{i}^{*}$ is independent of $a_{j}, \beta$ must be zero. Similar arguments can be used for the case when $p_{i}^{*}$ is independent of $c_{j}$.

Proof of Proposition 2: The proof is composed of four parts.

\section{Part 1:}

By (7),

$$
\mathbf{p}^{*}=\frac{\mathbf{a}+\mathbf{c}}{2}-\frac{\beta}{2}\left[(2-\beta) \mathbf{I}_{n}-2 \delta \mathbf{G}\right]^{-1}(\mathbf{a}-\mathbf{c}) .
$$

Differentiating with respect to a yields

$$
\frac{\partial \mathbf{p}^{*}}{\partial \mathbf{a}}=\underbrace{\frac{1}{2} \mathbf{I}_{n}-\frac{\beta}{2}\left[(2-\beta) \mathbf{I}_{n}-2 \delta \mathbf{G}\right]^{-1}}_{: \mathbf{\Omega}^{1}}=\frac{1}{2} \mathbf{I}_{n}-\frac{\beta}{2(2-\beta)} \mathbf{M}\left(\mathbf{G}, \frac{2 \delta}{2-\beta}\right) .
$$

The above equation implies that

$$
\begin{aligned}
\frac{\partial p_{i}^{*}}{\partial a_{j}} & =\Omega_{i j}^{1}=\frac{1}{2} \mathbf{i d}_{\{i=j\}}-\frac{\beta}{2(2-\beta)} m_{i j}\left(\mathbf{G}, \frac{2 \delta}{2-\beta}\right) \\
& = \begin{cases}\frac{1}{2}-\frac{\beta}{2(2-\beta)} m_{i i}\left(\mathbf{G}, \frac{2 \delta}{2-\beta}\right) & i=j ; \\
-\frac{\beta}{2(2-\beta)} m_{i j}\left(\mathbf{G}, \frac{2 \delta}{2-\beta}\right) & i \neq j .\end{cases}
\end{aligned}
$$

The off-diagonal entries are negative, i.e., $\frac{\partial p_{i}^{*}}{\partial a_{j}}<0$ if $i \neq j$, and the diagonal entry $\Omega_{i i}^{1}=\frac{1}{2}-$ $\frac{\beta}{2(2-\beta)} m_{i i}\left(\mathbf{G}, \frac{2 \delta}{2-\beta}\right)<1 / 2$ for any $i$.

Recall that the eigenvalues of matrix $\Omega^{1}$ are

$$
\frac{1}{2}-\frac{\beta}{2\left(2-\beta-2 \delta \lambda_{i}(\mathbf{G})\right)}=\frac{1-\beta-\delta \lambda_{i}(\mathbf{G})}{\left(2-\beta-2 \delta \lambda_{i}(\mathbf{G})\right)}
$$

where $\lambda_{i}(\mathbf{G}), i=1,2, \cdots, n$ are eigenvalues of $\mathbf{G}$. By Assumption 1,

$$
\left(1-\beta-\delta \lambda_{i}(\mathbf{G})\right)>0, \text { and }\left(2-\beta-2 \delta \lambda_{i}(\mathbf{G})\right)=\beta+2\left(1-\beta-\delta \lambda_{i}(\mathbf{G})\right)>0, \text { for all } i
$$


As a consequence, all the eigenvalues of matrix $\Omega^{1}$ are positive. Therefore, $\Omega^{1}$ is a positive definite matrix, and all the diagonal entries are strictly positive, i.e., $\frac{\partial p_{i}^{*}}{\partial a_{i}}=\Omega_{i i}^{1}>0$.

Thus, we have

$$
\frac{\partial p_{i}^{*}}{\partial a_{i}}=\Omega_{i i}^{1}=\frac{1}{2}-\frac{\beta}{2(2-\beta)} m_{i i}\left(\mathbf{G}, \frac{2 \delta}{2-\beta}\right) \in(0,1 / 2) .
$$

Moreover for $i \neq j$, by positive definiteness of $\Omega^{1},\left|\Omega_{i j}^{1}\right|<\sqrt{\Omega_{i i}^{1} \Omega_{j j}^{1}}<\sqrt{1 / 2 \cdot 1 / 2}=1 / 2$. Therefore,

$$
\frac{\partial p_{i}^{*}}{\partial a_{j}}=\Omega_{i j}^{1}=-\frac{\beta}{2(2-\beta)} m_{i i}\left(\mathbf{G}, \frac{2 \delta}{2-\beta}\right) \in(-1 / 2,0),
$$

for $i \neq j$.

Part 2:

By (7), we have

$$
\mathbf{p}^{*}=\frac{\mathbf{a}+\mathbf{c}}{2}-\frac{\beta}{2}\left[(2-\beta) \mathbf{I}_{n}-2 \delta \mathbf{G}\right]^{-1}(\mathbf{a}-\mathbf{c}) .
$$

Differentiating with respect to c yields

$$
\frac{\partial \mathbf{p}^{*}}{\partial \mathbf{c}}=\underbrace{\frac{1}{2} \mathbf{I}_{n}+\frac{\beta}{2}\left[(2-\beta) \mathbf{I}_{n}-2 \delta \mathbf{G}\right]^{-1}}_{: \Omega^{2}}=\frac{1}{2} \mathbf{I}_{n}+\frac{\beta}{2(2-\beta)} \mathbf{M}\left(\mathbf{G}, \frac{2 \delta}{2-\beta}\right) .
$$

Component-wise, we can rewrite the above as:

$$
\begin{aligned}
\frac{\partial p_{i}^{*}}{\partial c_{j}} & =\Omega_{i j}^{2}=\frac{1}{2} \mathbf{i d}_{\{i=j\}}+\frac{\beta}{2(2-\beta)} m_{i j}\left(\mathbf{G}, \frac{2 \delta}{2-\beta}\right) \\
& = \begin{cases}\frac{1}{2}+\frac{\beta}{2(2-\beta)} m_{i i}\left(\mathbf{G}, \frac{2 \delta}{2-\beta}\right) & i=j ; \\
\frac{\beta}{2(2-\beta)} m_{i j}\left(\mathbf{G}, \frac{2 \delta}{2-\beta}\right) & i \neq j ;\end{cases}
\end{aligned}
$$

Clearly, every entry of $\Omega^{2}$ is positive. More specifically, for $i=j$,

$$
\frac{\partial p_{i}^{*}}{\partial c_{i}}=\Omega_{i i}^{2}=\frac{1}{2}+\frac{\beta}{2(2-\beta)} m_{i i}\left(\mathbf{G}, \frac{2 \delta}{2-\beta}\right)=1-\Omega_{i i}^{1} \in(1 / 2,1),
$$

by (22). For $i \neq j$, we obtain

$$
\frac{\partial p_{i}^{*}}{\partial c_{j}}=\Omega_{i j}^{2}=\frac{\beta}{2(2-\beta)} m_{i i}\left(\mathbf{G}, \frac{2 \delta}{2-\beta}\right)=-\Omega_{i j}^{1} \in(0,1 / 2)
$$

by (23).

\section{Part 3:}


Differentiating with respect to $\delta$ in $(7)$, we obtain

$$
\frac{\partial \mathbf{p}^{*}}{\partial \delta}=-\beta\left[(2-\beta) \mathbf{I}_{n}-2 \delta \mathbf{G}\right]^{-2} \mathbf{G}(\mathbf{a}-\mathbf{c})
$$

Recall that $\beta>0,\left[(2-\beta) \mathbf{I}_{n}-2 \delta \mathbf{G}\right]^{-1} \succeq \mathbf{0}, \mathbf{G} \succeq \mathbf{0},(\mathbf{a}-\mathbf{c}) \succeq \mathbf{0}$. Therefore, $\frac{\partial \mathbf{p}^{*}}{\partial \delta} \preceq \mathbf{0}$, i.e., $\frac{\partial p_{i}^{*}}{\partial \delta} \leq 0$ for any $i$.

Differentiating with respect to $\delta$ in $(7)$ yields

$$
\frac{\partial \mathbf{p}^{*}}{\partial \beta}=-\frac{1}{2}\left[(2-\beta) \mathbf{I}_{n}-2 \delta \mathbf{G}\right]^{-1}(\mathbf{a}-\mathbf{c})-\frac{\beta}{2}\left[(2-\beta) \mathbf{I}_{n}-2 \delta \mathbf{G}\right]^{-2}(\mathbf{a}-\mathbf{c}) .
$$

Clearly $\frac{\partial \mathbf{p}^{*}}{\partial \beta} \preceq \mathbf{0}$ because $\beta>0,\left[(2-\beta) \mathbf{I}_{n}-2 \delta \mathbf{G}\right]^{-1} \succeq \mathbf{0},\left[(2-\beta) \mathbf{I}_{n}-2 \delta \mathbf{G}\right]^{-2} \succeq \mathbf{0},(\mathbf{a}-\mathbf{c}) \succeq \mathbf{0}$.

Part 4:

Since $\mathrm{G}^{\prime} \succ \mathrm{G}$, we have

$$
\begin{aligned}
& {\left[(2-\beta) \mathbf{I}_{n}-2 \delta \mathbf{G}^{\prime}\right]^{-1}-\left[(2-\beta) \mathbf{I}_{n}-2 \delta \mathbf{G}\right]^{-1} } \\
= & {\left[(2-\beta) \mathbf{I}_{n}-2 \delta \mathbf{G}^{\prime}\right]^{-1} 2 \delta \underbrace{\left(\mathbf{G}^{\prime}-\mathbf{G}\right)}_{\succeq \mathbf{0}}\left[(2-\beta) \mathbf{I}_{n}-2 \delta \mathbf{G}\right]^{-1} } \\
\succeq & \mathbf{0} .
\end{aligned}
$$

As a consequence, by (7),

$$
\begin{aligned}
\mathbf{p}^{*}\left(\mathbf{G}^{\prime}\right)-\mathbf{p}^{*}(\mathbf{G}) & =\left\{\frac{\mathbf{a}+\mathbf{c}}{2}-\frac{\beta}{2}\left[(2-\beta) \mathbf{I}_{n}-2 \delta \mathbf{G}^{\prime}\right]^{-1}(\mathbf{a}-\mathbf{c})\right\}-\left\{\frac{\mathbf{a}+\mathbf{c}}{2}-\frac{\beta}{2}\left[(2-\beta) \mathbf{I}_{n}-2 \delta \mathbf{G}\right]^{-1}(\mathbf{a}-\mathbf{c})\right\} \\
& =-\frac{\beta}{2} \underbrace{\left[(2-\beta) \mathbf{I}_{n}-2 \delta \mathbf{G}^{\prime}\right]^{-1}-\left[(2-\beta) \mathbf{I}_{n}-2 \delta \mathbf{G}\right]^{-1}}_{\succeq \mathbf{0}}(\mathbf{a}-\mathbf{c}) \preceq \mathbf{0} .
\end{aligned}
$$

In other words, $\mathbf{p}^{*}\left(\mathbf{G}^{\prime}\right) \preceq \mathbf{p}^{*}(\mathbf{G})$.

Proof of Proposition 3: Given the prices $\mathbf{p}=\left(\mathbf{p}^{A}, \mathbf{p}^{B}\right)$, we let $D_{i}^{k}(\mathbf{p})=\mathbb{E}\left[1_{\left\{\eta_{i}=A\right\}}\right]$ denote consumer $i$ 's probability of buying product $k=A, B$. Then

$$
\begin{aligned}
D_{i}^{A}(\mathbf{p}) & =\operatorname{Pr}\left(v_{i}^{A}+\delta \sum_{j} g_{i j} D_{j}^{A}(\mathbf{p})-p_{i}^{A}+\epsilon_{i}^{A} \geq v_{i}^{B}+\delta \sum_{j} g_{i j} D_{j}^{B}(\mathbf{p})-p_{i}^{B}+\epsilon_{i}^{B}\right) \\
& =\operatorname{Pr}\left(\epsilon_{i}^{A}-\epsilon_{i}^{B} \geq-\left(\left(v_{i}^{A}+\delta \sum_{j} g_{i j} D_{j}^{A}(\mathbf{p})-p_{i}^{A}\right)-\left(v_{i}^{B}+\delta \sum_{j} g_{i j} D_{j}^{B}(\mathbf{p})-p_{i}^{B}\right)\right)\right) \\
& =\frac{1}{2}+\frac{\left(\left(v_{i}^{A}+\delta \sum_{j} g_{i j} D_{j}^{A}(\mathbf{p})-p_{i}^{A}\right)-\left(v_{i}^{B}+\delta \sum_{j} g_{i j} D_{j}^{B}(\mathbf{p})-p_{i}^{B}\right)\right.}{2 t} .
\end{aligned}
$$

where in the last step we use the fact that $\epsilon_{i}^{A}-\epsilon_{i}^{B}$ is uniformly distributed on the interval $[-t, t]$, 
and $v_{i}^{A}=v_{i}^{B}$. Note that $D_{i}^{A}(\mathbf{p})+D_{i}^{B}(\mathbf{p})=1$ by full market coverage. So, in matrix term, we have

$$
\mathbf{D}^{A}=\frac{1}{2} \mathbf{1}_{n}+\frac{1}{2 t}\left[\delta \mathbf{G}\left(2 \mathbf{D}^{A}-\mathbf{1}_{n}\right)-\mathbf{p}^{A}+\mathbf{p}^{B}\right]
$$

or

$$
\mathbf{D}^{A}=\frac{1}{2} \mathbf{1}_{n}+\left[\mathbf{I}-\frac{\delta}{t} \mathbf{G}\right]^{-1} \frac{1}{2 t}\left(-\mathbf{p}^{A}+\mathbf{p}^{B}\right) .
$$

Similarly, we have

$$
\mathbf{D}^{B}=\frac{1}{2} \mathbf{1}_{n}-\left[\mathbf{I}-\frac{\delta}{t} \mathbf{G}\right]^{-1} \frac{1}{2 t}\left(-\mathbf{p}^{A}+\mathbf{p}^{B}\right) .
$$

The profit of firm $A$ is given by

$$
\pi^{A}=\left\langle\mathbf{D}^{A}, \mathbf{p}^{A}\right\rangle=\left\langle\frac{1}{2} \mathbf{1}_{n}+\left[\mathbf{I}-\frac{\delta}{t} \mathbf{G}\right]^{-1} \frac{1}{2 t}\left(-\mathbf{p}^{A}+\mathbf{p}^{B}\right), \mathbf{p}^{A}\right\rangle
$$

Since $t-\delta \lambda_{1}(\mathbf{G})>0$, the matrix $\left[\mathbf{I}-\frac{\delta}{t} \mathbf{G}\right]^{-1}$ is positive definite, hence the profit $\pi^{A}$ is strictly concave in $\mathbf{p}^{A}$. The FOCs are both necessary and sufficient for profit maximization, therefore we have

$$
\mathbf{D}^{A}+\left(\frac{\partial \mathbf{D}^{A}}{\partial \mathbf{p}^{A}}\right)^{\prime} \mathbf{p}^{A}=\mathbf{0}
$$

Similar expression is obtained for firm B. After plugging the expressions of $\mathbf{D}^{A}$ and $\mathbf{D}^{B}$, we solve these simultaneous equations and obtain that the equilibrium price vectors are

$$
\mathbf{p}^{A *}=\mathbf{p}^{B *}=t \mathbf{1}_{n}-\delta \mathbf{G}^{\prime} \mathbf{1}_{n} .
$$

In other words, the price charged to each consumer $i$ is equal to: $p_{i}^{*}=t-\delta d_{i}$. Moreover, the equilibrium demand vectors are $\mathbf{D}^{A *}=\mathbf{D}^{B *}=\frac{1}{2} \mathbf{1}_{n}$. Proposition 3 follows directly.

Proof of Proposition 4: By Theorem E.1, we have $\mathbf{x}^{*}(\mathbf{G} ; \beta, \delta):=\phi^{E X}(\mathbf{G} ; \beta, \delta)(\mathbf{a}-\mathbf{c})$. Therefore,

$$
\frac{\partial \mathbf{x}^{*}(\mathbf{G} ; \beta, \delta)}{\partial \mathbf{a}}=-\frac{\partial \mathbf{x}^{*}(\mathbf{G} ; \beta, \delta)}{\partial \mathbf{c}}=\phi^{E X}(\mathbf{G} ; \beta, \delta) \succeq \mathbf{0} .
$$

Similarly, by direct differentiation, we have

$$
\frac{\partial \mathbf{x}^{*}(\mathbf{G} ; \beta, \delta)}{\partial \beta}=\frac{\partial \phi^{E X}(\mathbf{G} ; \beta, \delta)}{\partial \beta}(\mathbf{a}-\mathbf{c}) .
$$

and

$$
\frac{\partial \mathbf{x}^{*}(\mathbf{G} ; \beta, \delta)}{\partial \delta}=\frac{\partial \phi^{E X}(\mathbf{G} ; \beta, \delta)}{\partial \delta}(\mathbf{a}-\mathbf{c}) .
$$

Moreover $\frac{\partial \mathbf{x}^{*}(\mathbf{G} ; \beta, \delta)}{\partial \delta} \succeq \mathbf{0}$. 
Note that

$$
\mathbf{x}^{*}(\mathbf{G} ; \beta, \delta)=\left[(1+\beta) \mathbf{I}_{n}-\delta \mathbf{G}\right]^{-1}\left(\frac{\mathbf{a}+\mathbf{c}}{c}+\frac{\beta}{2}\left[(2-\beta) \mathbf{I}_{n}-2 \delta \mathbf{G}\right]^{-1}(\mathbf{a}-\mathbf{c})\right) .
$$

Since both terms $\left[(1+\beta) \mathbf{I}_{n}-\delta \mathbf{G}\right]^{-1}$ and $\left[(2-\beta) \mathbf{I}_{n}-2 \delta \mathbf{G}\right]^{-1}$ are increasing in $\delta$ and $\mathbf{G}, \mathbf{x}^{*}(\mathbf{G} ; \beta, \delta)$ is increasing in $\delta$ and $\mathbf{G}$.

Proof of Corollary 3: These results just follow from direct differentiations with respect to the parameters $\beta, \delta$ and the fact that $\mathbf{C S}^{*}(\mathbf{G} ; \beta, \delta)$ is monotone in equilibrium consumption $\mathbf{x}^{*}(\mathbf{G} ; \beta, \delta)$.

Proof of Proposition 5: These results just follow from direct differentiations with respect to the parameters $\beta, \delta$. Moreover,

$$
\left.\frac{\partial \phi^{P T}}{\partial \beta}=\frac{-2(1-\delta z)}{(1+\beta-\delta z)^{2}(2-\beta-2 \delta z)^{3}}\left\{\left(\beta-\frac{1-\delta z}{2}\right)^{2}+\frac{3}{4}(1-\delta z)^{2}\right)\right\}
$$

It is easy to check that $\left.\frac{\partial \phi^{P T}(z)}{\partial \beta}\right|_{z=\lambda_{i}(\mathbf{G})}<0$, where $\lambda_{i}(\mathbf{G})$ is the eigenvalue value of $\mathbf{G}$. Hence, $\frac{\partial \phi^{P T}(\mathbf{G} ; \beta, \delta)}{\partial \beta}$ is a negative definite matrix; consequently, the sign

$$
\frac{\partial \mathbf{\Pi}^{*}(\mathbf{G} ; \beta, \delta)}{\partial \beta}=\left\langle(\mathbf{a}-\mathbf{c}), \frac{\partial \phi^{P T}(\mathbf{G} ; \beta, \delta)}{\partial \beta}(\mathbf{a}-\mathbf{c})\right\rangle
$$

is negative. The sign of $\Pi^{*}\left(\mathbf{G}^{\prime} ; \beta, \delta\right)-\Pi^{*}(\mathbf{G} ; \beta, \delta)$ in general is ambiguous by Proposition 7 .

Proof of Proposition 6: By Theorem E.1, the equilibrium firm profit is given by:

$$
\boldsymbol{\Pi}^{*}(d ; \beta, \delta)=n \phi^{P T}(d ; \beta, \delta)=n \frac{(1-\delta d)(1-\beta-\delta d)}{(1+\beta-\delta d)(2-\beta-2 \delta d)^{2}} .
$$

It is easily verified that:

$$
\frac{\partial \boldsymbol{\Pi}^{*}(d ; \beta, \delta)}{\partial \beta}=-n \frac{2(1-d \delta)\left[(1-d \delta)^{2}-(1-d \delta) \beta+\beta^{2}\right]}{(2-\beta-2 \delta d)^{3}(1+\beta-\delta d)^{2}}<0
$$

since $(1-d \delta)^{2}-(1-d \delta) \beta+\beta^{2}=\left(1-d \delta-\frac{\beta}{2}\right)^{2}+\frac{3}{4} \beta^{2}>0$. On the other hand,

$$
\frac{\partial \Pi^{*}(d ; \beta, \delta)}{\partial \delta}=-n d \frac{d\left[\beta^{3}+3(1-d \delta)^{2} \beta-2(1-d \delta)^{3}\right]}{(2-\beta-2 \delta d)^{3}(1+\beta-\delta d)^{2}} .
$$

Note that $\beta^{3}+3(1-d \delta)^{2} \beta-2(1-d \delta)^{3}>0$ if and only if $\beta>\beta^{*}(1-d \delta)$, where $\beta^{*} \approx 0.596072$ is the unique real root of $\beta^{3}+3 \beta-2$. Therefore,

$$
\frac{\partial \Pi^{*}(d ; \beta, \delta)}{\partial \delta}>0 \text { if and only if } \beta<\beta^{*}(1-d \delta) .
$$


Next, we investigate the impact of the degree $d$ on the equilibrium profit. Intuitively, this should be the same as increasing $\delta$ since:

$$
\frac{\partial \boldsymbol{\Pi}^{*}(d ; \beta, \delta)}{\partial d}=n(a-c)^{2} \frac{-\delta\left(\beta^{3}+3(1-d \delta)^{2} \beta-2(1-d \delta)^{3}\right)}{(2-\beta-2 \delta d)^{3}(1+\beta-\delta d)^{2}} .
$$

We observe that, when $\delta>0$,

$$
\frac{\partial \Pi^{*}(d ; \beta, \delta)}{\partial d}>0 \text { if and only if } \frac{\partial \Pi^{*}(d ; \beta, \delta)}{\partial \delta}>0 \text { if and only if } \beta<\beta^{*}(1-d \delta) .
$$

Therefore, the sign of $\partial \Pi^{*} / \partial d$ could be positive or negative, depending on the parameters.

Proof of Proposition 7: First we have the following Taylor expansion for $\phi^{P T}$ for small $\delta$ :

$$
\phi^{P T}(z ; \beta, \delta)=\frac{(1-\beta)}{(1+\beta)(2-\beta)^{2}}+\delta \frac{\left(2-3 \beta-\beta^{3}\right)}{(1+\beta)^{2}(2-\beta)^{3}} z+\mathcal{O}\left(\delta^{2}\right) .
$$

Hence, by (E.1) in Theroem E.1, we obtain

$$
\phi^{P T}(\mathbf{G} ; \beta, \delta)=\frac{(1-\beta)}{(1+\beta)(2-\beta)^{2}} \mathbf{I}_{n}+\delta \frac{\left(2-3 \beta-\beta^{3}\right)}{(1+\beta)^{2}(2-\beta)^{3}} \mathbf{G}+\mathcal{O}\left(\delta^{2}\right) .
$$

As a consequence, we have the following Taylor expansion for the equilibrium profit for small $\delta$ :

$$
\begin{aligned}
\boldsymbol{\Pi}^{*} & =\left\langle(\mathbf{a}-\mathbf{c}), \phi^{P T}(\mathbf{G} ; \beta, \delta)(\mathbf{a}-\mathbf{c})\right\rangle \\
& =\frac{(1-\beta)}{(1+\beta)(2-\beta)^{2}}\langle(\mathbf{a}-\mathbf{c}),(\mathbf{a}-\mathbf{c})\rangle+\delta \frac{\left(2-3 \beta-\beta^{3}\right)}{(1+\beta)^{2}(2-\beta)^{3}}\langle(\mathbf{a}-\mathbf{c}), \mathbf{G}(\mathbf{a}-\mathbf{c})\rangle+\mathcal{O}\left(\delta^{2}\right) .
\end{aligned}
$$

Now we proceed to establish the statement of the proposition. From the above Taylor expansions, we find that

$$
\boldsymbol{\Pi}\left(\mathbf{G}^{\prime}\right)-\boldsymbol{\Pi}(\mathbf{G})=\delta \frac{\left(2-3 \beta-\beta^{3}\right)}{(1+\beta)^{2}(2-\beta)^{3}}\left\langle(\mathbf{a}-\mathbf{c}),\left(\mathbf{G}^{\prime}-\mathbf{G}\right)(\mathbf{a}-\mathbf{c})\right\rangle+\mathcal{O}\left(\delta^{2}\right) .
$$

Observe that $\left\langle(\mathbf{a}-\mathbf{c}),\left(\mathbf{G}^{\prime}-\mathbf{G}\right)(\mathbf{a}-\mathbf{c})\right\rangle>0$ as $\mathbf{G}^{\prime} \succ \mathbf{G}$ by assumption. The cubic polynomial $2-3 \beta-\beta^{3}$ is decreasing in $\beta$ and it has a unique real root at $\beta^{*}=\sqrt[3]{1+\sqrt{2}}-\frac{1}{\sqrt[3]{1+\sqrt{2}}} \approx 0.596072$. Therefore, $2-3 \beta-\beta^{3}>0$ if and only if $\beta<\beta^{*}$. This cutoff value $\beta^{*}$ determines the sign of $\frac{\partial \Pi}{\partial \delta}$ around $\delta=0$. In other words, for a fixed $\beta>0$, the sign of $\Pi\left(\mathbf{G}^{\prime}\right)-\boldsymbol{\Pi}(\mathbf{G})$ is solely determined by the sign of $\operatorname{sign}\left\{\beta^{*}-\beta\right\}$ for $\delta>0$ but close to 0 .

Now suppose that we pick any number, say $\beta_{1}=0.4<\beta^{*}$. In this case, we can find a $\delta_{1}>0$ small enough such that $\Pi\left(\mathbf{G}^{\prime}\right)-\left.\boldsymbol{\Pi}(\mathbf{G})\right|_{\left(\beta_{1}, \delta_{1}\right)}>0$. Similarly, if we pick another number, say $\beta_{2}=0.7>\beta^{*}$, we can find $\delta_{2}$ small enough such that $\Pi\left(\mathbf{G}^{\prime}\right)-\left.\Pi(\mathbf{G})\right|_{\left(\beta_{2}, \delta_{2}\right)}>0$. The rest just follows from the continuity argument. 
Proof of Theorem 3: By equation (26), we have

$$
\Pi^{*}=\frac{(1-\beta)}{(1+\beta)(2-\beta)^{2}}\langle(\mathbf{a}-\mathbf{c}),(\mathbf{a}-\mathbf{c})\rangle+\delta \frac{\left(2-3 \beta-\beta^{3}\right)}{(1+\beta)^{2}(2-\beta)^{3}}\langle(\mathbf{a}-\mathbf{c}), \mathbf{G}(\mathbf{a}-\mathbf{c})\rangle+\mathcal{O}\left(\delta^{2}\right) .
$$

Since there are only a finite number of networks with $n$ nodes, there exists a small number $\bar{\delta}$ such that for $\delta<\bar{\delta}$, the leading term of $\Pi^{*}$ is determined by sign of $2-3 \beta-\beta^{3}$ and the number $\langle(\mathbf{a}-\mathbf{c}), \mathbf{G}(\mathbf{a}-\mathbf{c})\rangle$. Hence,

$$
\operatorname{sign}\left\{\Pi^{*}\left(\mathbf{G}^{\prime} ; \beta, \delta\right)-\Pi^{*}(\mathbf{G} ; \beta, \delta)\right\}=\operatorname{sign}\left\{\beta^{*}-\beta\right\}, \text { whenever } \mathbf{G}^{\prime} \succ \mathbf{G} \text {. }
$$

Proof of Theorem 4: Let $\mathbf{p}^{* A}=\mathbf{p}^{* B}=p^{u} \mathbf{1}_{n}$ be the unique symmetry uniform price. Now suppose firm A unilaterally deviates and decreases his price by $\Delta \mathbf{p}^{* A}=\Delta p^{A} \mathbf{1}_{n}$ (lowers the price by $\Delta p^{A}$ for every consumer), i.e., $\mathbf{p}^{* A}$ becomes equal to $\mathbf{p}^{*}-\Delta \mathbf{p}^{* A}$. It has two effects. On one hand, the price margins per unit are lower. This gives rise to the total marginal loss

$$
\approx\left\langle\mathbf{x}^{*}, \boldsymbol{\Delta} \mathbf{p}^{* A}\right\rangle=\left\langle\mathbf{M}^{+}\left(\mathbf{a}-p^{u} \mathbf{1}_{n}\right), \Delta \mathbf{p}^{* A}\right\rangle=\left\langle\mathbf{M}^{+}\left(\mathbf{a}-p^{u} \mathbf{1}_{n}\right), \Delta p^{A} \mathbf{1}_{n}\right\rangle .
$$

On the other hand, there is marginal benefit due to demand enhancing, which is $\approx\left\langle\Delta \mathbf{x}^{\mathbf{A}}, p^{u} \mathbf{1}_{n}-\right.$ $\mathbf{c}\rangle$. The change in consumption for $A$ due to lower $\mathbf{p}^{* A}$ is $\Delta \mathbf{x}^{\mathbf{A}}=\frac{\mathbf{M}^{+}+\mathbf{M}^{-}}{2} \Delta \mathbf{p}^{* A}=\frac{\mathbf{M}^{+}+\mathbf{M}^{-}}{2} \Delta p^{A} \mathbf{1}_{n}$. In equilibrium both effects must cancel out, i.e.,

$$
\left\langle\mathbf{M}^{+}\left(\mathbf{a}-p^{u} \mathbf{1}_{n}\right), \Delta p^{A} \quad \mathbf{1}_{n}\right\rangle=\left\langle\frac{\mathbf{M}^{+}+\mathbf{M}^{-}}{2} \Delta p^{A} \mathbf{1}_{n}, p^{u} \mathbf{1}_{n}-\mathbf{c}\right\rangle, \text { for any } \Delta p^{A} \in \mathbf{R} .
$$

As a consequence,

$$
\left\langle\mathbf{M}^{+}\left(\mathbf{a}-p^{u} \mathbf{1}_{n}\right), \mathbf{1}_{n}\right\rangle=\left\langle\frac{\mathbf{M}^{+}+\mathbf{M}^{-}}{2} \mathbf{1}_{n}, p^{u} \mathbf{1}_{n}-\mathbf{c}\right\rangle .
$$

Plugging in $\mathbf{M}^{+}$and $\mathbf{M}^{-}$and simplifying yields the theorem.

Proof of Proposition 8: The first part of the proposition is obvious since when either $G$ is a regular network, or $\beta=0$, or $\delta=0$, the equilibrium price in Theorem 1 is actually the same for every player and is equal to the uniform price. As a result, the firms' profit and consumer surplus are the same under both price regimes.

Let us now focus on the second part of the proposition where $G$ is not a regular network, and $\beta \neq 0$, and $\delta \neq 0$. For that, assume that $\delta$ is close to zero so that it is sufficient to prove the result for Taylor expansion expressions of $\Delta \Pi$ and $\Delta C S$. We expand the profit and surplus under two the pricing schemes up to the second order terms of $\delta$, and evaluate the difference.

First we consider the case with discriminatory pricing. Recall that the equilibrium pricing is given by Theorem 1 and the welfare results are shown in Theorem E. 1 in Appendix E. To derive 
the Taylor expansion, we first note that:

$$
\begin{aligned}
& \mathbf{M}^{+}=[(1+\beta) \mathbf{I}-\delta \mathbf{G}]^{-1}=\frac{1}{(1+\beta)}\left[\mathbf{I}+\frac{\delta \mathbf{G}}{(1+\beta)}+\frac{\delta^{2} \mathbf{G}^{2}}{(1+\beta)^{2}}+\mathcal{O}\left(\delta^{3}\right)\right] \\
& \mathbf{M}^{-}=[(1-\beta) \mathbf{I}-\delta \mathbf{G}]^{-1}=\frac{1}{(1-\beta)}\left[\mathbf{I}+\frac{\delta \mathbf{G}}{(1-\beta)}+\frac{\delta^{2} \mathbf{G}^{2}}{(1-\beta)^{2}}+\mathcal{O}\left(\delta^{3}\right)\right]
\end{aligned}
$$

Since we assume that $\mathbf{c}=\mathbf{0}, \mathbf{a}=\mathbf{1}_{n}$, by Theorem $\mathbf{1}$, we have:

$$
\begin{aligned}
\mathbf{p}^{*} & =\left(\frac{\mathbf{a}+\mathbf{c}}{2}\right)-\frac{\beta}{2}\left[(2-\beta) \mathbf{I}_{n}-2 \delta \mathbf{G}\right]^{-1}(\mathbf{a}-\mathbf{c}) \\
& =\left[\frac{1-\beta}{2-\beta} \mathbf{I}-\frac{\beta \delta \mathbf{G}}{(2-\beta)^{2}}-\frac{2 \beta \delta^{2} \mathbf{G}^{2}}{(2-\beta)^{3}}\right] \mathbf{1}_{n}+\mathcal{O}\left(\delta^{3}\right)
\end{aligned}
$$

The equilibrium demand is given by: $\mathbf{x}^{*}=\mathbf{M}^{+}\left(\mathbf{a}-\mathbf{p}^{*}\right)$. Using (27), we can compute the Taylor expansions for consumer surplus and firm profit as follows:

$$
\begin{aligned}
C S^{*} & =(1+\beta)\left\langle\mathbf{x}^{*}, \mathbf{x}^{*}\right\rangle=(1+\beta)\left\langle\mathbf{M}^{+}\left(\mathbf{a}-\mathbf{p}^{*}\right), \mathbf{M}^{+}\left(\mathbf{a}-\mathbf{p}^{*}\right)\right\rangle \\
& =\frac{n}{(2-\beta)^{2}(1+\beta)}\left[1+\frac{2(1+\beta)^{2}}{(2-\beta)(1+\beta)} \delta A d 1+\frac{\left(12+16 \beta^{2}+2 \beta^{3}+\beta^{4}\right)}{(2-\beta)^{2}(1+\beta)^{2}} \delta^{2} A d 2\right]+\mathcal{O}\left(\delta^{3}\right)(28) \\
\Pi^{*} & =\left\langle\mathbf{x}^{*},\left(\mathbf{p}^{*}-\mathbf{c}\right)\right\rangle=\left\langle\mathbf{M}^{+}\left(\mathbf{a}-\mathbf{p}^{*}\right),\left(\mathbf{p}^{*}-\mathbf{c}\right)\right\rangle \\
& =\frac{n}{(2-\beta)^{2}(1+\beta)}\left[(1-\beta)+\frac{2-3 \beta-\beta^{3}}{(2-\beta)(1+\beta)} \delta A d 1+\frac{2\left(2-4 \beta-4 \beta^{3}-\beta^{4}\right)}{(2-\beta)^{2}(1+\beta)^{2}} \delta^{2} A d 2\right]+\mathcal{O}\left(\delta^{3}\right)
\end{aligned}
$$

where $A d 1 \equiv \frac{1}{n} \sum d_{i}$ and $A d 2 \equiv \frac{1}{n} \sum d_{i}^{2}$.

Next we consider the uniform pricing. Since we assume that $\mathbf{c}=\mathbf{0}, \mathbf{a}=\mathbf{1}_{n}$, by Theorem 4 , the uniform price is given by:

$$
p^{u}=\frac{\left\langle\mathbf{1}_{n},\left(\mathbf{M}^{+} \mathbf{a}+\frac{\mathbf{M}^{+}+\mathbf{M}^{-}}{2} \mathbf{c}\right)\right\rangle}{\left\langle\mathbf{1}_{n},\left(\mathbf{M}^{+} \mathbf{1}_{n}+\frac{\mathbf{M}^{+}+\mathbf{M}^{-}}{2} \mathbf{1}_{n}\right)\right\rangle}=\frac{\left\langle\mathbf{1}_{n},\left(\mathbf{M}^{+} \mathbf{1}_{n}+\frac{\mathbf{M}^{+}+\mathbf{M}^{-}}{2} \mathbf{1}_{n}\right)\right\rangle}{\left\langle\mathbf{1}_{n},\left(\mathbf{M}^{+} \mathbf{1}_{n}+\frac{\mathbf{M}^{+}+\mathbf{M}^{-}}{2} \mathbf{1}_{n}\right)\right\rangle} .
$$

Let $\mathbf{x}^{u}=\mathbf{M}^{+}\left(\mathbf{a}-p^{u} \mathbf{1}_{n}\right)$ denote the equilibrium demand under uniform pricing. We can simplify the consumer surplus and write it as follows:

$$
\begin{aligned}
C S^{u}= & (1+\beta)\left\langle\mathbf{x}^{u}, \mathbf{x}^{u}\right\rangle=(1+\beta)\left\langle\mathbf{M}^{+}\left(\mathbf{a}-p^{u} \mathbf{1}_{n}\right), \mathbf{M}^{+}\left(\mathbf{a}-p^{u} \mathbf{1}_{n}\right)\right\rangle \\
= & \frac{n}{(2-\beta)^{2}(1+\beta)}\left[1+\frac{2(1+\beta)^{2}}{(2-\beta)(1+\beta)} \delta A d 1\right. \\
& \left.+\frac{\left(12-16 \beta+19 \beta^{2}-7 \beta^{3}\right) A d 2+\left(4 \beta-3 \beta^{2}-7 \beta^{3}-\beta^{4}-\beta^{5}\right)(A d 1)^{2}}{(2-\beta)^{2}(1+\beta)^{2}(1-\beta)} \delta^{2}\right]+\mathcal{O}\left(\delta^{3}\right)(29)
\end{aligned}
$$

where in the last step we use the Taylor expansions of $\mathbf{M}^{+}$and $\mathbf{M}^{-}$to simplify $p^{u}$. As a result, 
the difference in consumer surplus between the two pricing regimes is equal to:

$$
\Delta C S=C S^{u}-C S^{*}=\operatorname{Var}(d) \frac{n \beta\left(-4+7 \beta+\beta^{3}\right)}{(1-\beta)(1+\beta)^{2}(2-\beta)^{4}} \delta^{2}+\mathcal{O}\left(\delta^{3}\right) .
$$

where $\operatorname{Var}(d)=A d 2-(A d 1)^{2}=\left(\frac{1}{n} \sum d_{i}^{2}\right)-\left(\frac{1}{n} \sum d_{i}\right)^{2} \geq 0$.

In a similar way, we can compute the profit under uniform pricing $\Pi^{u}=\left\langle\mathbf{x}^{u},\left(p^{u} \mathbf{1}_{n}-\mathbf{c}\right)\right\rangle$ and the difference in profits between the two price regimes is given by:30

$$
\Delta \Pi=\Pi^{u}-\Pi^{*}=\operatorname{Var}(d) \frac{n \beta^{2}(1-2 \beta)}{\left(1-\beta^{2}\right)(2-\beta)^{4}} \delta^{2}+\mathcal{O}\left(\delta^{3}\right) .
$$

Clearly, when the variance of the degrees in the network is equal to zero, i.e., $\operatorname{Var}(d)=0$, so that $d_{i}=d$ for every $i$, the network $G$ is regular and $\Delta \Pi=\Delta C S=0$

Assume $\operatorname{Var}(d)>0$ (equivalently $\mathbf{G}$ is not regular). From these two expressions (30) and (31), when $\delta$ is close to zero, then

$$
\Delta \Pi \gtreqless 0 \Longleftrightarrow \beta \lesseqgtr \frac{1}{2}
$$

and

$$
\Delta C S \gtreqless 0 \Longleftrightarrow \beta \gtreqless 0.548
$$

Indeed, to obtain the second result, it suffices to observe that $-4+7 \beta+\beta^{3}$ is strictly increasing in $\beta$ and 0.548 is the unit real root of $-4+7 \beta+\beta^{3}$. By continuity, these two results have to be true for values of $\delta$ that are small but greater than zero.

\footnotetext{
${ }^{30}$ Details about intemediate steps are available upon request.
} 


\title{
Online Appendix Competitive Pricing Strategies in Social Networks
}

\author{
By Ying-Ju Chen ${ }^{1}$, Yves Zenou ${ }^{2}$ and Junjie Zhou ${ }^{3}$
}

March 12, 2018

In this Online Appendix, we derive some matrix operations and define the Katz-Bonacich centrality (Appendix A), deal with the single representative consumer case (Appendix B), provide additional results for the duopoly case (Appendix $C$ ), illustrate our results for specific networks (Appendix D), characterize the total welfare of the economy (Appendix E) and provide the proofs of all the results in this Appendix (Appendix F).

\section{A Matrix notation and Katz-Bonacich centrality}

Matrix notation. Let us have some notation for the matrices and vectors in general. In this paper, $\mathbf{I}_{k}$ is the $k \times k$ identity matrix, $\mathbf{J}_{p q}$ is the $p \times q$ matrix with 1 's, and $\mathbf{1}_{n}=\mathbf{J}_{n 1}$ is a column vector with 1s:

$$
\mathbf{I}_{k}=\left[\begin{array}{ccc}
1 & \cdots & 0 \\
& \ddots & \\
0 & \cdots & 1
\end{array}\right]_{k \times k}, \quad \mathbf{J}_{p q}=\left[\begin{array}{ccc}
1 & \cdots & 1 \\
\vdots & \ddots & \vdots \\
1 & \cdots & 1
\end{array}\right]_{p \times q}, \mathbf{1}_{n}=\left[\begin{array}{c}
1 \\
\vdots \\
1
\end{array}\right]_{n \times 1} .
$$

The inner product of two vectors $\mathbf{x}=\left(x_{1}, \cdots, x_{n}\right)^{\prime}$ and $\mathbf{y}=\left(y_{1}, \cdots, y_{n}\right)^{\prime}$ in $\mathbf{R}^{n}$ is denoted as $\langle\mathbf{x}, \mathbf{y}\rangle=\sum_{i} x_{i} y_{i}$. We use $\mathbf{0}$ to denote the zero matrix with suitable dimensions. For any two matrices $\mathbf{H}$ and $\mathbf{D}, \mathbf{H} \preceq(\succeq) \mathbf{D}$ if component-wise $h_{i j} \leq(\geq) d_{i j}$ for all $i, j$, where $\left\{h_{11}, \ldots, h_{m n}\right\}$ 's and $\left\{d_{11}, \ldots, d_{m n}\right\}$ 's are the components of $\mathbf{H}$ and $\mathbf{D}$, respectively. Consequently, $\mathbf{H}$ is a positive matrix if $\mathbf{H} \succeq \mathbf{0}$. $\mathbf{H}^{\prime}$ represents the transpose of a matrix $\mathbf{H}$. A square symmetric matrix $\mathbf{H}$ is called positive definite if all of its eigenvalues are strictly positive.

Katz-Bonacich centrality. Let us define the Katz-Bonacich centrality. Denote by $\lambda_{1}(\mathbf{G})$ the spectral radius of matrix G. Since G is a nonnegative matrix, by the Perron-Frobenius Theorem it is also equal to its largest eigenvalue.

Definition A.1. Assume $0 \leq \delta<1 / \lambda_{1}(\mathbf{G})$. Then, for any vector $\mathbf{a}=\left(a_{1}, \cdots, a_{n}\right)^{\prime} \in \mathbf{R}^{n}$, the KatzBonacich centrality vector with weight a is defined as:

$$
\mathbf{b}(\mathbf{G}, \delta, \mathbf{a}):=\mathbf{M}(\mathbf{G}, \delta) \mathbf{a},
$$

\footnotetext{
${ }^{1}$ School of Business and Management \& School of Engineering, The Hong Kong University of Science and Technology; e-mail: imchen@ust.hk.

${ }^{2}$ Department of Economics, Monash University, IFN and CEPR. Email: yves.zenou@monash.edu.

${ }_{3}^{3}$ Department of Economics, National University of Singapore; e-mail: zhoujjozoo1@gmail.com.
} 
where

$$
\mathbf{M}(\mathbf{G}, \delta)=[\mathbf{I}-\delta \mathbf{G}]^{-1}=\mathbf{I}+\sum_{k \geq 1} \delta^{k} \mathbf{G}^{k} .
$$

Let $b_{i}(\mathbf{G}, \delta, \mathbf{a})$ be the ith entry of $\mathbf{b}(\mathbf{G}, \delta, \mathbf{a})$. Let $m_{i j}(\mathbf{G}, \delta)$ be the ij entry of $\mathbf{M}(\mathbf{G}, \delta)$. Then,

$$
b_{i}(\mathbf{G}, \delta, \mathbf{a})=\sum_{j} m_{i j}(\mathbf{G}, \delta) a_{j} .
$$

\section{B Single representative consumer case}

In this appendix, we consider the case without the network effects. We investigate this benchmark using the single representative consumer setup $(n=1)$. The consumer's utility function is:

$$
u\left(x^{A}, x^{B}\right)+I=a^{A} x^{A}+a^{B} x^{B}-\left(\frac{1}{2}\left(x^{A}\right)^{2}+\left(x^{B}\right)^{2}+\beta x^{A} x^{B}\right)+I .
$$

Here $I$ is the composite good (i.e., it serves as the numeraire). The parameter $\beta$ satisfies $|\beta|<1$.

Given this utility function, the consumer's problem is

$$
\max _{\left\{x^{A}, x^{B}, I\right\}}\left\{U\left(x^{A}, x^{B}, I\right) \mid \text { s.t. } p^{A} x^{A}+p^{B} x^{B}+I \leq w\right\},
$$

or equivalently,

$$
\max _{\left\{x^{A}, x^{B}\right\}}\left\{\left(a^{A}-p^{A}\right) x^{A}+\left(a^{B}-p^{B}\right) x^{B}-\left(\frac{1}{2}\left(x^{A}\right)^{2}+\left(x^{B}\right)^{2}+\beta x^{A} x^{B}\right)\right\} .
$$

Let

$$
\Psi=\left[\begin{array}{ll}
1 & \beta \\
\beta & 1
\end{array}\right], \Psi^{-1}=\frac{1}{1-\beta^{2}}\left[\begin{array}{cc}
1 & -\beta \\
-\beta & 1
\end{array}\right]
$$

The first-order conditions can be written as

$$
\left[\begin{array}{l}
a^{A}-p^{A} \\
a^{B}-p^{B}
\end{array}\right]=\Psi\left[\begin{array}{l}
x^{A} \\
x^{B}
\end{array}\right] \text {, or }\left[\begin{array}{l}
x^{A} \\
x^{B}
\end{array}\right]=\Psi^{-1}\left[\begin{array}{c}
a^{A}-p^{A} \\
a^{B}-p^{B}
\end{array}\right]=\frac{1}{1-\beta^{2}}\left[\begin{array}{cc}
1 & -\beta \\
-\beta & 1
\end{array}\right]\left[\begin{array}{c}
a^{A}-p^{A} \\
a^{B}-p^{B}
\end{array}\right] \text {. }
$$

These are the demand functions for the differentiated Bertrand competition model.

Monopoly setup. In this case, a monopoly firm sells both products. Let $c^{A}$ and $c^{B}$ be the marginal costs of products $A$ and $B$. The monopoly firm's objective is

$$
\max _{\left\{p^{A}, p^{B}\right\}}\left[\begin{array}{ll}
p^{A}-c^{A} & p^{B}-c^{B}
\end{array}\right] \frac{1}{1-\beta^{2}}\left[\begin{array}{cc}
1 & -\beta \\
-\beta & 1
\end{array}\right]\left[\begin{array}{c}
a^{A}-p^{A} \\
a^{B}-p^{B}
\end{array}\right] .
$$


Let $\bar{p}^{A}, \bar{p}^{B}$ be the solutions. The first-order conditions for the joint maximization lead to

$$
\frac{1}{1-\beta^{B}}\left[\begin{array}{cc}
1 & -\beta \\
-\beta & 1
\end{array}\right]\left[\begin{array}{c}
a^{A}+c^{A}-2 \bar{p}^{A} \\
a^{B}+c^{B}-2 \bar{p}^{B}
\end{array}\right]=\left[\begin{array}{l}
0 \\
0
\end{array}\right]
$$

Since $\Psi^{-1}$ is invertible, we have

$$
\left[\begin{array}{c}
a^{A}+c^{A}-2 \bar{p}^{A} \\
a^{B}+c^{B}-2 \bar{p}^{B}
\end{array}\right]=\left[\begin{array}{l}
0 \\
0
\end{array}\right], \Longrightarrow\left[\begin{array}{c}
\bar{p}^{A} \\
\bar{p}^{B}
\end{array}\right]=\left[\begin{array}{c}
\frac{a^{A}+c^{A}}{{ }^{2}} \\
\frac{a^{B}+c^{B}}{2}
\end{array}\right]
$$

Duopoly setup. Now suppose that the two prices are controlled by two different firms. Let

$$
\begin{aligned}
& \pi^{A}\left(p^{A}, p^{B}\right)=\left(p^{A}-c^{A}\right) x^{A}=\frac{1}{1-\beta^{2}}\left(p^{A}-c^{A}\right)\left(\left(a^{A}-p^{A}\right)-\beta\left(a^{B}-p^{B}\right)\right), \\
& \pi^{B}\left(p^{A}, p^{B}\right)=\left(p^{B}-c^{B}\right) x^{B}=\frac{1}{1-\beta^{2}}\left(p^{B}-c^{B}\right)\left(\left(a^{B}-p^{B}\right)-\beta\left(a^{A}-p^{A}\right)\right) .
\end{aligned}
$$

The first-order conditions for the Nash equilibrium are:

$$
\begin{aligned}
& \frac{\partial \pi^{A}\left(p^{A}, p^{B}\right)}{\partial p^{A}}=0, \Longrightarrow\left(\left(a^{A}-p^{A}\right)-\beta\left(a^{B}-p^{B}\right)\right)=\left(p^{A}-c^{A}\right), \\
& \frac{\partial \pi^{B}\left(p^{A}, p^{B}\right)}{\partial p^{B}}=0, \Longrightarrow\left(\left(a^{B}-p^{B}\right)-\beta\left(a^{A}-p^{A}\right)\right)=\left(p^{B}-c^{B}\right) .
\end{aligned}
$$

In matrix forms, we obtain that: $\left[\begin{array}{cc}2 & -\beta \\ -\beta & 2\end{array}\right]\left[\begin{array}{c}p^{A} \\ p^{B}\end{array}\right]=\left[\begin{array}{c}a^{A}+c^{A}-\beta a^{B} \\ a^{B}+c^{B}-\beta a^{A}\end{array}\right]$. Therefore, the equilibrium prices are

$$
\left[\begin{array}{c}
p^{* A} \\
p^{* B}
\end{array}\right]=\left[\begin{array}{cc}
2 & -\beta \\
-\beta & 2
\end{array}\right]^{-1}\left[\begin{array}{c}
a^{A}+c^{A}-\beta a^{B} \\
a^{B}+c^{B}-\beta a^{A}
\end{array}\right]=\frac{1}{4-\beta^{2}}\left[\begin{array}{c}
2\left(a^{A}+c^{A}-\beta a^{B}\right)+\beta\left(a^{B}+c^{B}-\beta a^{A}\right) \\
2\left(a^{B}+c^{B}-\beta a^{A}\right)+\beta\left(a^{A}+c^{A}-\beta a^{B}\right)
\end{array}\right] .
$$

When $a^{A}=a^{A}=a, c^{A}=c^{B}=c$, the duopoly prices are $\left[\begin{array}{l}p^{* A} \\ p^{* B}\end{array}\right]=\left[\begin{array}{c}\frac{(1-\beta) a+c}{2-\beta} \\ \frac{(1-\beta) a+c}{2-\beta}\end{array}\right]$. When $\beta>0$, for substitute products, the duopoly price $\frac{(1-\beta) a+c}{2-\beta}$ is lower than the monopoly price $\frac{(a+c)}{2}$. When $\beta<0$, for complements products, the duopoly price $\frac{(1-\beta) a+c}{2-\beta}$ is actually higher than the monopoly price $\frac{(a+c)}{2}$. This results have obvious counter-parts for $l \geq 2$ products.

\section{Additional results under duopoly competition}

This section collects some auxiliary results that are not reported in the main text. 


\section{C.1 Equilibrium prices when network effects are small}

Here, we consider an asymptotic regime with sufficiently small network effects, i.e., when $\delta$ is sufficiently small. Using Taylor expansions, we can obtain more transparent expressions of the equilibrium prices.

Theorem C.1. Suppose Assumptions 1 and 2 hold. When $\delta$ is sufficiently small, the equilibrium price $\mathbf{p}^{*}$ is equal to:

$$
\mathbf{p}^{*}=\frac{(1-\beta) \mathbf{a}+\mathbf{c}}{2-\beta}-\frac{\delta \beta}{(2-\beta)^{2}} \mathbf{G}(\mathbf{a}-\mathbf{c})+\mathcal{O}\left(\delta^{2}\right) .
$$

In other words, for each consumer $i$,

$$
p_{i}^{A}=p_{i}^{B}=\frac{(1-\beta) a_{i}+c_{i}}{2-\beta}-\frac{\delta \beta}{(2-\beta)^{2}} \sum_{j} g_{i j}\left(a_{j}-c_{j}\right)+\mathcal{O}\left(\delta^{2}\right) .
$$

Furthermore, if we assume that $a_{i}=a, c_{i}=c$ for all $i$ (i.e. Assumption 3 holds), then

$$
p_{i}^{A}=p_{i}^{B}=\frac{(1-\beta) a+c}{2-\beta}-\frac{\delta \beta(a-c)}{(2-\beta)^{2}} d_{i}+\mathcal{O}\left(\delta^{2}\right)
$$

where $d_{i}=\sum_{j} g_{i j}$ is the degree of consumer $i$.

When $\delta$ is small, we obtain an intuitive pricing rule based on the degree of each consumer. In equation (C.2), a consumer with a higher degree will always be charged a lower price. In this respect, the firms compensate consumers that are well-connected because their consumptions will boost other consumers' willingness to pay. Again this result has no counterpart in the monopoly case. More generally, this means that firms, when setting their price, do not need to know the whole network but, as in Fainmesser and Galeotti (2016), only need to know the degree of each consumer.

\section{C.2 Collusive pricing and merger analysis}

Previously we study the competitive pricing between firms. Now we analyze the case where the two firms determine the prices $\mathbf{p}^{A}$ and $\mathbf{p}^{B}$ jointly to maximize their total profits. This could be the case when two firms merge into a single firm that controls both $\mathbf{p}^{A}$ and $\mathbf{p}^{B}$. In such a benchmark, the optimal collusive prices solve

$$
\max _{\left\{\mathbf{p}^{A}, \mathbf{p}^{B}\right\}}\left\{\Pi^{A}(\mathbf{p})+\Pi^{B}(\mathbf{p})\right\} .
$$

Let $\overline{\mathbf{p}}^{A}, \overline{\mathbf{p}}^{B}$ be the solutions of this program.

Lemma C.1. Suppose that the two firms jointly determine their prices. If Assumption 1 holds, the 
corresponding collusive prices are given by

$$
\overline{\mathbf{p}}^{A}=\frac{\mathbf{a}^{A}+\mathbf{c}^{A}}{2}, \quad \overline{\mathbf{p}}^{B}=\frac{\mathbf{a}^{B}+\mathbf{c}^{B}}{2} .
$$

Lemma C.1 shows that the collusive price for a consumer $i=1, \ldots, n$ only depends on this consumer's marginal utility and the marginal cost of product $t$. It is, however, independent of the network structure $\mathbf{G}$, the strength of network effect $\delta$, and the degree of substitution $\beta$ between the two products. Moreover, if both firms are symmetric (i.e., Assumption 2 holds), then the collusive prices are also symmetric, and $\overline{\mathbf{p}}^{A}=\overline{\mathbf{p}}^{B}=\frac{\mathbf{a}+\mathbf{c}}{2}$. This network-independent result in the two-product case shares some similarity with the outcomes in the monopoly setting studied by Bloch and Quérou (2013) and Candogan et al. (2012), which have only one product. It also gives us a useful benchmark for the equilibrium prices with competition.

\section{C.3 Complementary goods}

Under Assumption 1, the mathematical formulas for equilibrium analysis carry over line to line for complementary goods $(\beta<0)$. However, some of the signs of comparative statics are reversed due to different interdependent pattern between two goods. For example, when $\beta<0$, we still have

$$
\frac{\partial \mathbf{x}^{B}}{\partial \mathbf{a}^{A}}=-\frac{\partial \mathbf{x}^{B}}{\partial \mathbf{p}^{A}}=\frac{\mathbf{M}^{+}-\mathbf{M}^{-}}{2}
$$

but the sign is different:

$$
\frac{\partial \mathbf{x}^{B}}{\partial \mathbf{a}^{A}}=-\frac{\partial \mathbf{x}^{B}}{\partial \mathbf{p}^{A}} \succeq \mathbf{0}
$$

since $\beta \leq 0$ implies that $\mathbf{M}^{+} \succeq \mathbf{M}^{-}$. In other words, when two goods $A$ and $B$ are complements, then if the price of good $A$ rises, each consumer will consume less of product $B$. When product $A$ becomes more attractive, the consumption for good $B$ also rises. Clearly, these predictions are in contrast with the substitutable case $(\beta<0)$.

Another difference is the price implication. The second term in the price $\mathbf{p}^{*}$ in Theorem 1 is now positive as $\beta<0$. Therefore, each consumer need to pay a price premium, on top of $\frac{a+c}{2}$. The proof is omitted.

Proposition C.1. The competitive prices $\mathbf{p}^{*}$ characterized by Theorem 1 exhibit influence-based discount when goods are substitutable (i.e. the more central the consumers are in the network, the lower is their price paid for consuming goods $A$ and $B$ ) but exhibit influence-based premium when goods are complements (i.e. the more central the consumers are in the network, the higher is their price paid for consuming goods $A$ and $B)$.

Potentially this proposition has some empirical implications. Specifically, it ties the product characteristics with the firms' competitive pricing strategies. One might use consumer surveys to identify whether the products are substitutable or complementary separately, and then draw 
the connection between consumer survey results and the observable price quotes.

\section{C.4 Asymmetric firms}

So far, we have assumed that each consumer has the same intrinsic marginal utility for different products. This assumption implies that the equilibrium prices for different products are also the same for a fixed consumer. In this subsection, we remove this symmetry assumption and solve for equilibrium prices with heterogeneous intrinsic marginal utilities and marginal costs. For simplicity, we concentrate on the duopoly case. We have the following result:

Theorem C.2. Suppose that Assumption 1 holds. Then, for any $\mathbf{a}^{A}, \mathbf{a}^{B}$ and $\mathbf{c}^{A}, \mathbf{c}^{B}$, there exists a unique equilibrium in prices $\left(\hat{\mathbf{p}}^{A}, \hat{\mathbf{p}}^{B}\right)$ that satisfies:

$$
\left\{\begin{array}{l}
\hat{\mathbf{p}}^{A}=\frac{\mathbf{a}^{A}+\mathbf{c}^{A}}{2}-\frac{\beta}{2(2-\beta)} \mathbf{b}\left(\mathbf{G}, \frac{2 \delta}{2-\beta^{\prime}},\left(\frac{\mathbf{a}^{A}+\mathbf{a}^{B}}{2}-\frac{\mathbf{c}^{A}+\mathbf{c}^{B}}{2}\right)\right)+\frac{\beta}{2(2+\beta)} \mathbf{b}\left(\mathbf{G}, \frac{2 \delta}{2+\beta^{\prime}},\left(\frac{\mathbf{a}^{A}-\mathbf{a}^{B}}{2}-\frac{\mathbf{c}^{A}-\mathbf{c}^{B}}{2}\right)\right), \\
\hat{\mathbf{p}}^{B}=\frac{\mathbf{a}^{B}+\mathbf{c}^{B}}{2}-\frac{\beta}{2(2-\beta)} \mathbf{b}\left(\mathbf{G}, \frac{2 \delta}{2-\beta},\left(\frac{\mathbf{a}^{A}+\mathbf{a}^{B}}{2}-\frac{\mathbf{c}^{A}+\mathbf{c}^{B}}{2}\right)\right)-\frac{\beta}{2(2+\beta)} \mathbf{b}\left(\mathbf{G}, \frac{2 \delta}{2+\beta^{\prime}},\left(\frac{\mathbf{a}^{A}-\mathbf{a}^{B}}{2}-\frac{\mathbf{c}^{A}-\mathbf{c}^{B}}{2}\right)\right) .
\end{array}\right.
$$

We see that the equilibrium prices depend of the average marginal willingness to pay for the products and on the position of the consumers in the network as captured by their Katz-Bonacich centralities. From Theorem C.2, we can express the price differential as follows:

$$
\frac{\hat{\mathbf{p}}^{A}-\hat{\mathbf{p}}^{B}}{2}=\frac{1}{2}\left(\frac{\mathbf{a}^{A}-\mathbf{a}^{B}}{2}+\frac{\mathbf{c}^{A}-\mathbf{c}^{B}}{2}\right)+\frac{\beta}{2(2+\beta)} \mathbf{b}\left(\mathbf{G}, \frac{2 \delta}{2+\beta^{\prime}},\left(\frac{\mathbf{a}^{A}-\mathbf{a}^{B}}{2}-\frac{\mathbf{c}^{A}-\mathbf{c}^{B}}{2}\right)\right) .
$$

The above equation leads to an intriguing implication. Suppose that for all $i, a_{i}^{A}>a_{i}^{B}, c_{i}^{A}=c_{i}^{B}$, so product $A$ is more "attractive" than product $B$, then firm $A$ charges higher prices than firm $B$ due to its competitive advantage. Moreover, these price differentials are amplified when the network grows.

\section{Some specific networks}

Let us now illustrate our main results regarding equilibrium prices and their properties for some specific network structures. We will first illustrate the results obtained in Theorem 1 where we showed that price competition between two firms leads to the fact that, in equilibrium, the structure of the network and the intensity of network effects matter in the price determination of the goods. We will then illustrate the comparative statics results of Proposition 2, especially the impact on the density of the network on equilibrium prices. In all of the examples in this section, we assume that Assumptions 1 and 2 hold, and thus we can apply Theorem 1 to compute the equilibrium prices. In some cases, we will impose a stronger condition by replacing Assumption 2 with Assumption 3. 


\section{D.1 The dyad: complete graph with 2 nodes $\left(\mathrm{K}_{2}\right)$}

Let us start with the simplest possible network, the dyad (denoted by $\mathbf{K}_{2}$ ), which is the complete graph with only 2 consumers. It is displayed in Figure D.I

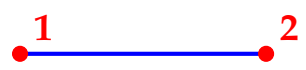

Figure D.1: The dyad

As stated above, we adopt Assumption 2 so that, for $i=1,2, a_{i}^{A}=a_{i}^{B}=a_{i}$ and $c_{i}^{A}=c_{i}^{B}=c_{i}$. Using Theorem 1 , we obtain the following equilibrium prices $\left(p_{1}^{A *}=p_{1}^{B *}=p_{1}^{*}\right.$ and $p_{2}^{A *}=p_{2}^{B *}=$ $\left.p_{2}^{*}\right): 4$

$$
\begin{aligned}
& {\left[(2-\beta)^{2}-4 \delta^{2}\right] \mathbf{p}^{*}=\left[(2-\beta)^{2}-4 \delta^{2}\right]\left[\begin{array}{l}
p_{1}^{*} \\
p_{2}^{*}
\end{array}\right] } \\
= & {\left[\begin{array}{cc}
(1-\beta)(2-\beta)-2 \delta^{2} & -\delta \beta \\
-\delta \beta & (1-\beta)(2-\beta)-2 \delta^{2}
\end{array}\right]\left[\begin{array}{l}
a_{1} \\
a_{2}
\end{array}\right]+\left[\begin{array}{cc}
(2-\beta)-2 \delta^{2} & \delta \beta \\
\delta \beta & (2-\beta)-2 \delta^{2}
\end{array}\right]\left[\begin{array}{l}
c_{1} \\
c_{2}
\end{array}\right] . }
\end{aligned}
$$

For the dyad, the network only plays a little role and thus firms do not discriminate consumers according to their location in the network. It is easily verified that, when the marginal cost $c_{1}$ for serving consumer 1 increases, equilibrium prices for both consumers increase $\left(\partial p_{1}^{*} / \partial c_{1}>0\right.$ and $\left.\partial p_{2}^{*} / \partial c_{1}>0\right)$. By contrast, when $a_{1}$, the marginal intrinsic value of consumer 1 increases, the equilibrium price for consumer 1 increases, but the price for consumer 2 decreases $\left(\partial p_{1}^{*} / \partial a_{1}>0\right.$ and $\left.\partial p_{2}^{*} / \partial a_{1}<0\right)$. Moreover, when $a_{1}=a_{2}=a, c_{1}=c_{2}=c$, we obtain:

$$
p_{1}^{*}=p_{2}^{*}=\frac{(1-\delta-\beta) a+(1-\delta) c}{2-2 \delta-\beta}
$$

Let us now present some numerical examples of this model min Table D.1. As can be seen, even for this very simple network, the comparative statics results are not trivial. We start with the first row when the parameters are: $a_{1}=3, a_{2}=4, c_{1}=c_{2}=1, \beta=0.4$ and $\delta=0.2$. Then, when we increase $a_{1}$ by 1 (second row), $p_{1}^{*}$ increases by 22.5 percent but $p_{2}^{*}$ decreases by only 1.6 percent. In other words, the effect of an increase of the marginal intrinsic value of consumer 1 has different impact on prices.

Next, suppose that we increase $c_{2}$ by 1 (third row). In this case, both prices increase, but $p_{1}^{*}$ increases by only 2.1 percent while $p_{2}^{*}$ increase by 31.2 percent. When $\beta$ increases or $\delta$ increases (columns four and five, respectively), both prices decrease. These signs and percentage changes of prices are consistent with Proposition 2. All these results depend on the value of the degree of substitution (or degree of product differentiation) $\beta$ between the two goods and that of the network externalities $\delta$.

${ }^{4}$ We assume that Assumption 1 holds, that is $1-\beta-\delta>0$. 


\begin{tabular}{|c|c|c|c|c|}
\hline$\left(a_{1}, a_{2}\right)$ & $\left(c_{1}, c_{2}\right)$ & $\beta$ & $\delta$ & $\left(p_{1}^{*}, p_{2}^{*}\right)$ \\
\hline$(3,4)$ & $(1, \mathbf{1})$ & 0.4 & 0.2 & $(1.633,2.033)$ \\
\hline$(\underline{\mathbf{4}}, 4)$ & $(\mathbf{1}, \mathbf{1})$ & 0.4 & 0.2 & $(2.000,2.000)$ \\
$(3,4)$ & $(\mathbf{1}, \underline{2})$ & 0.4 & 0.2 & $(1.667,2.667)$ \\
$(3,4)$ & $(1, \mathbf{1})$ & $\underline{\mathbf{0 . 5}}$ & 0.2 & $(1.498,1.866)$ \\
$(3,4)$ & $(1, \mathbf{1})$ & $\underline{0.4}$ & $\underline{\mathbf{0 . 3}}$ & $(1.545,1.955)$ \\
\hline
\end{tabular}

Table D.1: Equilibrium prices for different parameters for the dyad network. The underlined parameters in bold denote changes compared with the case on the first row.

\section{D.2 Regular graphs}

We now consider the family of regular graphs. A network $\mathbf{G}$ is regular of degree $d$ if each node has exactly $d$ neighbors, ${ }^{5}$ i.e., $\mathbf{G} \mathbf{1}_{n}=d \mathbf{1}_{n}$. Figure D.2 displays an example of a regular graph of degree 2. For simplicity, we adopt here Assumption 3, i.e., $a_{i}=a_{j}=a, c_{i}=c_{j}=c$.

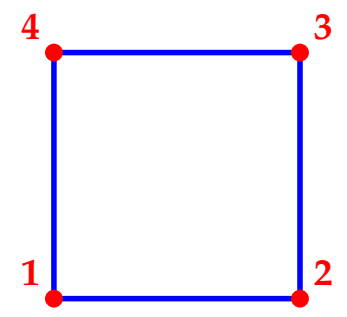

Figure D.2: A circle of four nodes $\mathbf{O}_{4}$, which is also a regular graph of degree 2.

By Theorem 1, we obtain the following equilibrium prices for an regular graph of degree $d: 6$

$$
\mathbf{p}^{*}=c+\left(\frac{1-d \delta-\beta}{2-2 d \delta-\beta}\right)(a-c) \mathbf{1}_{n}
$$

By differentiating this equation, we obtain $\frac{\partial p^{*}}{\partial a}>0, \frac{\partial p^{*}}{\partial c}>0$. Moreover,

$$
\frac{\partial p^{*}}{\partial \beta}<0, \operatorname{sign}\left\{\frac{\partial p^{*}}{\partial \delta}\right\}=-\operatorname{sign}\{\beta\}, \operatorname{sign}\left\{\frac{\partial p^{*}}{\partial d}\right\}=-\operatorname{sign}\{\beta \delta\} .
$$

When $\beta>0$ and $\delta>0$, the equilibrium price $p^{*}$ is decreasing in $\delta, \beta$, and the degree $d$. As above, these results are due to intensified competition between the two products when each of these parameters increases. In particular, our last result says that the more connected consumers are (e.g. by having a denser network), the lower is the price paid for consuming the two goods.

5The dyad network studied above is clearly a regular network of degree $d=1$.

${ }^{6}$ We assume that Assumption 1 holds, that is $1-\beta-2 \delta>0$. 


\section{D.3 The complete bipartite graph $\mathbf{K}_{p q}$}

Let us finally consider the complete bipartite graph, which is commonly used to model two-sided markets (see e.g. Ambrus and Argenziano (2009) and Jullien (2011)). In a complete bipartite graph $\mathbf{K}_{m q}$, there are two disjoint groups $M$ and $Q$ such that any node in $M$ is connected to any node in $Q$. Let $m=|M|$ and $q=|Q|$. Then, the network size satisfies $n=m+q$. The adjacency matrix of a complete bipartite graph is given by: $\mathbf{G}=\left[\begin{array}{cc}0 & \mathbf{J}_{m q} \\ \mathbf{J}_{q m} & 0\end{array}\right]$ where $\mathbf{J}_{m q}=\left[\begin{array}{ccc}1 & \cdots & 1 \\ \vdots & \ddots & \vdots \\ 1 & \cdots & 1\end{array}\right]_{m \times q}$. Figures D.3 and D.4 display two examples of bipartite networks for $m=1, q=5$ (Figure D.3) and $m=2, q=3$ (Figure D.4).

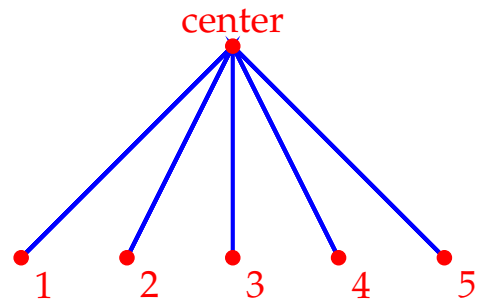

Figure D.3: A bipartite graph for $\mathbf{K}_{15}$

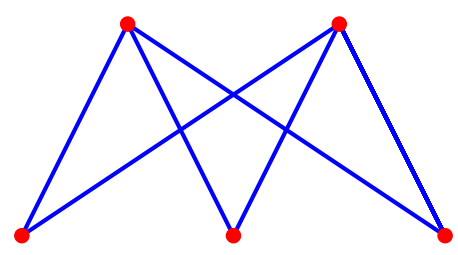

Figure D.4: A bipartite graph for $\mathbf{K}_{23}$.

For $\mathbf{K}_{p q}$, the adjacency matrix is

$$
\mathbf{G}=\left[\begin{array}{cc}
0 & \mathbf{J}_{p q} \\
\mathbf{J}_{q p} & 0
\end{array}\right]
$$

and

$$
\left[(2-\beta) \mathbf{I}_{n}-2 \delta \mathbf{G}\right]^{-1}=\frac{1}{(2-\beta)}\left[\begin{array}{cc}
{\left[\mathbf{I}_{p}+\frac{4 \delta^{2} q}{(2-\beta)^{2}-4 \delta^{2} q p} J_{p p}\right]} & \frac{2 \delta(2-\beta)}{(2-\beta)^{2}-4 \delta^{2} p q} J_{p q} \\
\frac{2 \delta(2-\beta)}{(2-\beta)^{2}-4 \delta^{2} p q} J_{q p} & {\left[\mathbf{I}_{q}+\frac{4 \delta^{2} p}{(2-\beta)^{2}-4 \delta^{2} q p} J_{q q}\right]}
\end{array}\right] .
$$

Using (7), we obtain

$$
\mathbf{p}^{*}=\frac{(1-\beta) \mathbf{a}+\mathbf{c}}{2-\beta}-\frac{\beta}{(2-\beta)\left((2-\beta)^{2}-4 \delta^{2} q p\right)}\left[\begin{array}{cc}
2 \delta^{2} q \mathbf{J}_{p p} & \delta(2-\beta) \mathbf{J}_{p q} \\
\delta(2-\beta) \mathbf{J}_{q p} & 2 \delta^{2} p \mathbf{J}_{q q}
\end{array}\right](\mathbf{a}-\mathbf{c}) .
$$

Moreover, under Assumption $3\left(a_{i}=a, c_{i}=c\right)$, this can be further simplified to:

$$
\mathbf{p}^{*}=\frac{(1-\beta) a+c}{2-\beta} \mathbf{1}_{n}-\frac{\beta(a-c)}{(2-\beta)\left((2-\beta)^{2}-4 \delta^{2} q p\right)}\left[\begin{array}{l}
\left(2 \delta^{2} p q+\delta(2-\beta) q\right) \mathbf{1}_{p} \\
\left(2 \delta^{2} p q+\delta(2-\beta) p\right) \mathbf{1}_{q}
\end{array}\right] .
$$


Therefore, the equilibrium prices can be expressed as:

$$
\mathbf{p}^{*}=\frac{(1-\beta) \mathbf{a}+\mathbf{c}}{2-\beta}-\frac{\beta}{(2-\beta)\left((2-\beta)^{2}-4 \delta^{2} q m\right)}\left[\begin{array}{cc}
2 \delta^{2} q \mathbf{J}_{m m} & \delta(2-\beta) \mathbf{J}_{m q} \\
\delta(2-\beta) \mathbf{J}_{q m} & 2 \delta^{2} p \mathbf{J}_{q q}
\end{array}\right](\mathbf{a}-\mathbf{c}) \text {. }
$$

Let us interpret equation (D.2). Consider two consumers $i_{1}, i_{2}$ in one group of the bipartite graph. We find that $p_{i_{1}}^{*}>p_{i_{2}}^{*}$ if and only if $a_{i_{1}}>a_{i_{2}}$. Thus, within each group, the consumer with a higher intrinsic valuation of the good will be charged a higher price. However, there is no clear comparison for the prices across different groups. Moreover, under Assumption 3 (i.e. $a_{i}=a, c_{i}=c$ ), the equilibrium prices can be written as (D.2). Therefore, in equilibrium, there are only two equilibrium prices, one for group $M$ (denoted by $p_{M}^{*}$ ), the other for group $Q$ (denoted by $p_{Q}^{*}$ ) where

$$
\left[\begin{array}{l}
p_{M}^{*} \\
p_{Q}^{*}
\end{array}\right]=\frac{1}{2-\beta}\left[\begin{array}{c}
(1-\beta) a+c-\frac{\beta(a-c)\left(2 \delta^{2} m q+\delta(2-\beta) q\right)}{(2-\beta)^{2}-4 \delta^{2} q m} \\
(1-\beta) a+c-\frac{\beta(a-c)\left(2 \delta^{2} m q+\delta(2-\beta) m\right)}{(2-\beta)^{2}-4 \delta^{2} q m}
\end{array}\right] .
$$

As a result, the price difference between group $P$ and $Q$ is equal to:

$$
p_{M}^{*}-p_{Q}^{*}=\frac{\beta \delta(2-\beta)(a-c)}{(2-\beta)\left((2-\beta)^{2}-4 \delta^{2} q m\right)} \times(m-q) .
$$

Thus, we obtain that

$$
p_{M}^{*}>p_{Q}^{*} \text { if and only if }|M|=m>|Q|=q .
$$

Applying this result to the star network (where $m=1$ ), we conclude that the consumer located in the center is charged with a lower price than the consumers located at the periphery.

\section{D.4 Star versus complete networks}

Consider the following two networks and let us analyze the impact of the network structure (density) on equilibrium prices. It can be seen that adding one link between nodes 2 and 3 in the star network $\mathbf{K}_{12}$ in Figure D.5 leads to the complete network $\mathbf{K}_{3}$ in Figure D.6.

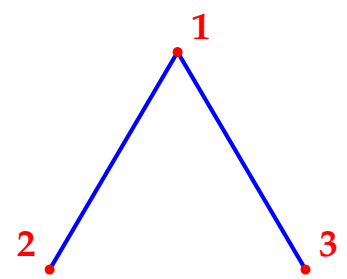

Figure D.5: $\operatorname{star} \mathbf{K}_{12}$

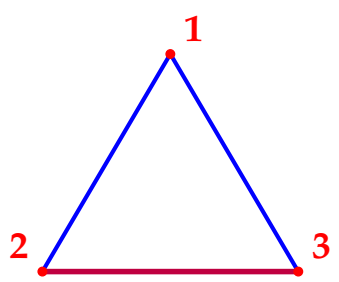

Figure D.6: $\mathbf{K}_{3}$

Using Proposition 2, the equilibrium price for every consumer in the complete network $\mathbf{K}_{3}$ is lower than in the star network $\mathbf{K}_{12}$ for any parameter value. This is because the network $\mathbf{K}_{3}$ 
is denser than the network $\mathbf{K}_{12}$. This can be seen by comparing the second and third columns in Table D.2 where we have calculated the equilibrium prices for these two networks for specific parameter values.

\begin{tabular}{|c|ccc|ccc|}
\hline network & \multicolumn{3}{|c|}{$\mathbf{K}_{12}$} & \multicolumn{3}{c|}{$\mathbf{K}_{3}$} \\
\hline$\left(a_{1}, a_{2}, a_{3}\right)$ & $p_{1}^{*}$ & $p_{2}^{*}$ & $p_{3}^{*}$ & $p_{1}^{*}$ & $p_{2}^{*}$ & $p_{3}^{*}$ \\
\hline$(3,3,3)$ & 1.764 & 1.790 & 1.790 & 1.754 & 1.754 & 1.754 \\
\hline$(4,3,3)$ & 2.172 & 1.777 & 1.777 & 2.162 & 1.739 & 1.739 \\
\hline$(3,4,3)$ & 1.751 & 2.200 & 1.788 & 1.739 & 2.162 & 1.739 \\
\hline$(3.03,3.01,3)$ & 1.776 & 1.794 & 1.790 & 1.766 & 1.758 & 1.753 \\
\hline
\end{tabular}

Table D.2: Equilibrium prices for two different networks. The parameters are $c_{i}=1, i=1,2,3$, $\beta=0.3$ and $\delta=0.12$.

Furthermore, for the complete network $\mathbf{K}_{3}$, the network position of every node is the same and, thus, a higher marginal utility $a_{i}$ means a higher price $p_{i}^{*}$ (see third row in Table D.2). For the star network $\mathbf{K}_{12}$, when all consumers have the same $a$, the consumer with higher (KatzBonacich) centrality will have a lower price. Indeed, the price for the center consumer 1 in $\mathbf{K}_{12}$ is $p_{1}^{*}=1.764$, which is lower than $p_{2}^{*}=p_{3}^{*}=1.790$. In the last row of Table D.2, consumer 1 has the largest marginal utility of consuming the product. When the network is the star $\mathbf{K}_{12}$, her price is the lowest. However, when consumers 2 and 3 form a link, i.e., when the network becomes complete, it will then be consumer 1 who will experience the highest price. This is because, in the star network, the central position of individual I "compensates" for her strong willingness to pay for the product. This is clearly not anymore the case in the complete network where 1 has no positional advantage and thus does not generate more network externalities than the other consumers. This highlights the key trade off that firms face when deciding on their prices. They have some monopoly power over consumers who have a strong preference for consuming the good but they also need to take into account how much network externalities each consumer generates, which is captured by the individual's network centrality.

\section{E Welfare characterization}

In this section, we determine the firms' equilibrium profits and consumer welfare. Let us define the following rational functions of $z$ :

$$
\begin{aligned}
\phi^{E X}(z ; \beta, \delta) & :=\frac{1-\delta z}{(1+\beta-\delta z)(2-\beta-2 \delta z)} \\
\phi^{P T}(z ; \beta, \delta) & :=\frac{(1-\delta z)(1-\beta-\delta z)}{(1+\beta-\delta z)(2-\beta-2 \delta z)^{2}}
\end{aligned}
$$




$$
\begin{aligned}
\phi^{C S}(z ; \beta, \delta): & =(1+\beta)\left[\frac{1-\delta z}{(1+\beta-\delta z)(2-\beta-2 \delta z)}\right]^{2}, \\
\phi^{T W}(z ; \beta, \delta) & :=\phi^{C S}(z)+2 \phi^{P T}(z) .
\end{aligned}
$$

Theorem E.1. Suppose Assumptions 1 and 2 hold. Then, the equilibrium consumption for each product is given by:

$$
\mathbf{x}^{*}(\mathbf{G} ; \beta, \delta):=\phi^{E X}(\mathbf{G} ; \beta, \delta)(\mathbf{a}-\mathbf{c})
$$

while each firm's equilibrium profit is equal to:

$$
\mathbf{\Pi}^{*}(\mathbf{G} ; \beta, \delta):=\left\langle(\mathbf{a}-\mathbf{c}), \phi^{P T}(\mathbf{G} ; \beta, \delta)(\mathbf{a}-\mathbf{c})\right\rangle .
$$

Furthermore, the total consumer surplus is equal to:

$$
\mathbf{C S}^{*}(\mathbf{G} ; \beta, \delta):=\left\langle(\mathbf{a}-\mathbf{c}), \phi^{C S}(\mathbf{G} ; \beta, \delta)(\mathbf{a}-\mathbf{c})\right\rangle .
$$

while the total welfare, defined as the sum of the consumer surplus and the equilibrium profit, is given by:

$$
\mathbf{T W}^{*}(\mathbf{G} ; \beta, \delta):=\left\langle(\mathbf{a}-\mathbf{c}), \phi^{T W}(\mathbf{G} ; \beta, \delta)(\mathbf{a}-\mathbf{c})\right\rangle .
$$

Remark 1. Note that $\phi^{E X}(\mathbf{G} ; \beta, \delta)$ should be interpreted as

$$
\left[\mathbf{I}_{n}-\delta \mathbf{G}\right] \times\left[(1+\beta) \mathbf{I}_{n}-\delta \mathbf{G}\right]^{-1} \times\left[(2-\beta) \mathbf{I}_{n}-2 \delta \mathbf{G}\right]^{-1} .
$$

using equation (E.1). Since the matrices $\left[\mathbf{I}_{n}-\delta \mathbf{G}\right],\left[(1+\beta) \mathbf{I}_{n}-\delta \mathbf{G}\right]^{-1}$ and $\left[(2-\beta) \mathbf{I}_{n}-2 \delta \mathbf{G}\right]^{-1}$ commute, the order of multiplications does not matter. Similar explanations for $\phi^{P T}(\mathbf{G} ; \beta, \delta), \phi^{C S}(\mathbf{G} ; \beta, \delta)$ and $\phi^{T W}(\mathbf{G} ; \beta, \delta)$ in Theorem E.1.

\section{F Proofs for the results in the online Appendix}

Proof of Theorem C.1: By (7), we obtain

$$
\mathbf{p}^{*}=\frac{\mathbf{a}+\mathbf{c}}{2}-\frac{\beta}{2}\left[(2-\beta) \mathbf{I}_{n}-2 \delta \mathbf{G}\right]^{-1}(\mathbf{a}-\mathbf{c}) .
$$

For small $\delta$, the inverse matrix can be expanded as follows:

$$
\left[(2-\beta) \mathbf{I}_{n}-2 \delta \mathbf{G}\right]^{-1}=\frac{1}{2-\beta}\left[\mathbf{I}_{n}-\frac{2 \delta}{(2-\beta)} \mathbf{G}\right]^{-1}=\frac{1}{2-\beta} \mathbf{I}_{n}+\frac{2 \delta}{(2-\beta)^{2}} \mathbf{G}+\mathcal{O}\left(\delta^{2}\right) .
$$


Hence,

$$
\begin{aligned}
\mathbf{p}^{*} & =\frac{\mathbf{a}+\mathbf{c}}{2}-\frac{\beta}{2}\left(\frac{1}{2-\beta} \mathbf{I}_{n}+\frac{2 \delta}{(2-\beta)^{2}} \mathbf{G}+\mathcal{O}\left(\delta^{2}\right)\right)(\mathbf{a}-\mathbf{c}) \\
& =\frac{(1-\beta) \mathbf{a}+\mathbf{c}}{2-\beta}+\delta\left(\frac{-\beta}{(2-\beta)^{2}} \mathbf{G}(\mathbf{a}-\mathbf{c})\right)+\mathcal{O}\left(\delta^{2}\right) .
\end{aligned}
$$

Specifically, for each consumer $i$, we have

$$
p_{i}^{A}=p_{i}^{B}=\frac{(1-\beta) a_{i}+c_{i}}{2-\beta}-\frac{\delta \beta}{(2-\beta)^{2}} \sum_{j} g_{i j}\left(a_{j}-c_{j}\right)+\mathcal{O}\left(\delta^{2}\right) .
$$

When $a_{i}=a, c_{i}=c$ for all $i$, we can simplify the above term further:

$$
p_{i}^{A}=p_{i}^{B}=\frac{(1-\beta) a+c}{2-\beta}-\frac{\delta \beta(a-c)}{(2-\beta)^{2}} d_{i}+\mathcal{O}\left(\delta^{2}\right) .
$$

Proof of Lemma C.1: We can express the joint profit as

$$
\begin{aligned}
\pi^{A}\left(\mathbf{p}^{A}, \mathbf{p}^{B}\right)+\pi^{B}\left(\mathbf{p}^{A}, \mathbf{p}^{B}\right) & =\left[\begin{array}{ll}
\mathbf{p}^{A}-\mathbf{c}^{A} & \mathbf{p}^{B}-\mathbf{c}^{B}
\end{array}\right]\left[\begin{array}{c}
x^{A}\left(\mathbf{p}^{A}, \mathbf{p}^{B}\right) \\
x^{B}\left(\mathbf{p}^{A}, \mathbf{p}^{B}\right)
\end{array}\right] \\
& =\left[\begin{array}{ll}
\mathbf{p}^{A}-\mathbf{c}^{A} & \mathbf{p}^{B}-\mathbf{c}^{B}
\end{array}\right]\left[\begin{array}{ll}
\frac{\mathbf{M}^{+}+\mathbf{M}^{-}}{2} & \frac{\mathbf{M}^{+}-\mathbf{M}^{-}}{2} \\
\frac{\mathbf{M}^{+}-\mathbf{M}^{-}}{2} & \frac{\mathbf{M}^{+}+\mathbf{M}^{-}}{2}
\end{array}\right]\left[\begin{array}{c}
\mathbf{a}^{A}-\mathbf{p}^{A} \\
\mathbf{a}^{B}-\mathbf{p}^{B}
\end{array}\right]
\end{aligned}
$$

The corresponding first-order conditions are

$$
\left[\begin{array}{ll}
\frac{\mathbf{M}^{+}+\mathbf{M}^{-}}{2} & \frac{\mathbf{M}^{+}-\mathbf{M}^{-}}{2} \\
\frac{\mathbf{M}^{+}-\mathbf{M}^{-}}{2} & \frac{\mathbf{M}^{+}+\mathbf{M}^{-}}{2}
\end{array}\right]\left[\begin{array}{c}
\mathbf{a}^{A}-\overline{\mathbf{p}}^{A} \\
\mathbf{a}^{B}-\overline{\mathbf{p}}^{B}
\end{array}\right]-\left[\begin{array}{ll}
\frac{\mathbf{M}^{+}+\mathbf{M}^{-}}{2} & \frac{\mathbf{M}^{+}-\mathbf{M}^{-}}{2} \\
\frac{\mathbf{M}^{+}-\mathbf{M}^{-}}{2} & \frac{\mathbf{M}^{+}+\mathbf{M}^{-}}{2}
\end{array}\right]^{\prime}\left[\begin{array}{c}
\overline{\mathbf{p}}^{A}-\mathbf{c}^{A} \\
\overline{\mathbf{p}}^{B}-\mathbf{c}^{B}
\end{array}\right]=0
$$

Notice that matrix $\left[\begin{array}{ll}\frac{\mathbf{M}^{+}+\mathbf{M}^{-}}{2} & \frac{\mathbf{M}^{+}-\mathbf{M}^{-}}{2} \\ \frac{\mathbf{M}^{+}-\mathbf{M}^{-}}{2} & \frac{\mathbf{M}^{+}+\mathbf{M}^{-}}{2}\end{array}\right]$ is symmetric. As a result, (F.1) can be simplified to

$$
\left[\begin{array}{ll}
\frac{\mathbf{M}^{+}+\mathbf{M}^{-}}{2} & \frac{\mathbf{M}^{+}-\mathbf{M}^{-}}{2} \\
\frac{\mathbf{M}^{+}-\mathbf{M}^{-}}{2} & \frac{\mathbf{M}^{+}+\mathbf{M}^{-}}{2}
\end{array}\right]\left[\begin{array}{c}
\mathbf{a}^{A}+\mathbf{c}^{A}-2 \overline{\mathbf{p}}^{A} \\
\mathbf{a}^{B}+\mathbf{c}^{B}-2 \overline{\mathbf{p}}^{B}
\end{array}\right]=\left[\begin{array}{l}
\mathbf{0} \\
\mathbf{0}
\end{array}\right]
$$

Recall that the eigenvalues of $\left[\begin{array}{ll}\frac{\mathbf{M}^{+}+\mathbf{M}^{-}}{2} & \frac{\mathbf{M}^{+}-\mathbf{M}^{-}}{2} \\ \frac{\mathbf{M}^{+}-\mathbf{M}^{-}}{2} & \frac{\mathbf{M}^{+}+\mathbf{M}^{-}}{2}\end{array}\right]$ are $\frac{\lambda_{i}(\mathbf{G})}{1 \pm \beta}, i=1, \cdots, n$, which are positive by Assumption 1. Hence, it is an invertible matrix. This then leads to:

$$
\left[\begin{array}{c}
\mathbf{a}^{A}+\mathbf{c}^{A}-2 \overline{\mathbf{p}}^{A} \\
\mathbf{a}^{B}+\mathbf{c}^{B}-2 \overline{\mathbf{p}}^{B}
\end{array}\right]=\left[\begin{array}{l}
\mathbf{0} \\
\mathbf{0}
\end{array}\right] \Longleftrightarrow\left[\begin{array}{c}
\overline{\mathbf{p}}^{A} \\
\overline{\mathbf{p}}^{B}
\end{array}\right]=\left[\begin{array}{c}
\frac{\mathbf{a}^{A}+\mathbf{c}^{A}}{2} \\
\frac{\mathbf{a}^{B}+\mathbf{c}^{B}}{2}
\end{array}\right]
$$


The result just follows.

Proof of Theorem C.2: In equilibrium, the following must hold:

$$
\left.\frac{\partial \Pi^{A}}{\partial \mathbf{p}^{A}}\right|_{\mathbf{p}^{A}=\hat{\mathbf{p}}^{A}}=\mathbf{0}, \text { and }\left.\frac{\partial \Pi^{B}}{\partial \mathbf{p}^{B}}\right|_{\mathbf{p}^{B}=\hat{\mathbf{p}}^{B}}=\mathbf{0}
$$

(Since second order conditions hold, these conditions are both necessary and sufficient to determine the equilibrium prices.) From (19) and (20), these first-order conditions can be written as

$$
\left\{\begin{array}{l}
\frac{\mathbf{M}^{+}+\mathbf{M}^{-}}{2}\left(\mathbf{a}^{A}-\hat{\mathbf{p}}^{A}\right)+\frac{\mathbf{M}^{+}-\mathbf{M}^{-}}{2}\left(\mathbf{a}^{B}-\hat{\mathbf{p}}^{B}\right)=\frac{\mathbf{M}^{+}+\mathbf{M}^{-}}{2}\left(\hat{\mathbf{p}}^{A}-\mathbf{c}^{A}\right), \\
\frac{\mathbf{M}^{+}-\mathbf{M}^{-}}{2}\left(\mathbf{a}^{A}-\hat{\mathbf{p}}^{A}\right)+\frac{\mathbf{M}^{+}+\mathbf{M}^{-}}{2}\left(\mathbf{a}^{B}-\hat{\mathbf{p}}^{B}\right)=\frac{\mathbf{M}^{+}+\mathbf{M}^{-}}{2}\left(\hat{\mathbf{p}}^{B}-\mathbf{c}^{B}\right) .
\end{array}\right.
$$

In matrix form, we have

$$
\left[\begin{array}{ll}
\frac{\mathbf{M}^{+}+\mathbf{M}^{-}}{2} & \frac{\mathbf{M}^{+}-\mathbf{M}^{-}}{2} \\
\frac{\mathbf{M}^{+}-\mathbf{M}^{-}}{2} & \frac{\mathbf{M}^{+}+\mathbf{M}^{-}}{2}
\end{array}\right]\left[\begin{array}{c}
\mathbf{a}^{A}-\hat{\mathbf{p}}^{A} \\
\mathbf{a}^{B}-\hat{\mathbf{p}}^{B}
\end{array}\right]=\left[\begin{array}{cc}
\frac{\mathbf{M}^{+}+\mathbf{M}^{-}}{2} & \mathbf{0} \\
\mathbf{0} & \frac{\mathbf{M}^{+}+\mathbf{M}^{-}}{2}
\end{array}\right]\left[\begin{array}{c}
\hat{\mathbf{p}}^{A}-\mathbf{c}^{A} \\
\hat{\mathbf{p}}^{B}-\mathbf{c}^{B}
\end{array}\right] .
$$

Taking the summation in (F.2) yields

$$
\mathbf{M}^{+}\left(\mathbf{a}^{A}+\mathbf{a}^{B}-\hat{\mathbf{p}}^{A}-\hat{\mathbf{p}}^{B}\right)=\frac{\mathbf{M}^{+}+\mathbf{M}^{-}}{2}\left(\hat{\mathbf{p}}^{A}+\hat{\mathbf{p}}^{B}-\mathbf{c}^{A}-\mathbf{c}^{B}\right)
$$

Therefore,

$$
\hat{\mathbf{p}}^{A}+\hat{\mathbf{p}}^{B}=\left(3 \mathbf{M}^{+}+\mathbf{M}^{-}\right)^{-1}\left\{2 \mathbf{M}^{+}\left(\mathbf{a}^{A}+\mathbf{a}^{B}\right)+\left(\mathbf{M}^{+}+\mathbf{M}^{-}\right)\left(\mathbf{c}^{A}+\mathbf{c}^{B}\right)\right\} .
$$

Plugging in $\mathbf{M}^{+}=\left[(1+\beta) \mathbf{I}_{n}-\delta \mathbf{G}\right]^{-1}, \quad \mathbf{M}^{-}=\left[(1-\beta) \mathbf{I}_{n}-\delta \mathbf{G}\right]^{-1}$, and simplifying it, we obtain that

$$
\begin{aligned}
\frac{\hat{\mathbf{p}}^{A}+\hat{\mathbf{p}}^{B}}{2} & =\left[(2-\beta) \mathbf{I}_{n}-2 \delta \mathbf{G}\right]^{-1}\left[\left((1-\beta) \mathbf{I}_{n}-\delta \quad \mathbf{G}\right) \frac{\mathbf{a}^{A}+\mathbf{a}^{B}}{2}+\left(\mathbf{I}_{n}-\delta \mathbf{G}\right) \frac{\mathbf{c}^{A}+\mathbf{c}^{B}}{2}\right] \\
& =\frac{\frac{\mathbf{a}^{A}+\mathbf{a}^{B}}{2}+\frac{\mathbf{c}^{A}+\mathbf{c}^{B}}{2}}{2}-\frac{\beta}{2(2-\beta)} \mathbf{b}\left(\mathbf{G}, \frac{2 \delta}{2-\beta},\left(\frac{\mathbf{a}^{A}+\mathbf{a}^{B}}{2}-\frac{\mathbf{c}^{A}+\mathbf{c}^{B}}{2}\right)\right) .
\end{aligned}
$$

Similarly, taking the difference in (F.2) yields

$$
\mathbf{M}^{-}\left(\mathbf{a}^{A}-\mathbf{a}^{B}-\hat{\mathbf{p}}^{A}+\hat{\mathbf{p}}^{B}\right)=\frac{\mathbf{M}^{+}+\mathbf{M}^{-}}{2}\left(\hat{\mathbf{p}}^{A}-\hat{\mathbf{p}}^{B}-\mathbf{c}^{A}+\mathbf{c}^{B}\right),
$$

and therefore

$$
\hat{\mathbf{p}}^{A}-\hat{\mathbf{p}}^{B}=\left(3 \mathbf{M}^{-}+\mathbf{M}^{+}\right)^{-1}\left\{2 \mathbf{M}^{-}\left(\mathbf{a}^{A}-\mathbf{a}^{B}\right)+\left(\mathbf{M}^{+}+\mathbf{M}^{-}\right)\left(\mathbf{c}^{A}-\mathbf{c}^{B}\right)\right\} .
$$

Plugging in $\mathbf{M}^{+}=\left[(1+\beta) \mathbf{I}_{n}-\delta \mathbf{G}\right]^{-1}, \quad \mathbf{M}^{-}=\left[(1-\beta) \mathbf{I}_{n}-\delta \mathbf{G}\right]^{-1}$ and simplifying it, the above 
equation can be rewritten as

$$
\begin{aligned}
\frac{\hat{\mathbf{p}}^{A}-\hat{\mathbf{p}}^{B}}{2} & =\left[(2+\beta) \mathbf{I}_{n}-2 \delta \mathbf{G}\right]^{-1}\left[\left((1+\beta) \mathbf{I}_{n}-\delta \quad \mathbf{G}\right) \frac{\mathbf{a}^{A}-\mathbf{a}^{B}}{2}+\left(\mathbf{I}_{n}-\delta \mathbf{G}\right) \frac{\mathbf{c}^{A}-\mathbf{c}^{B}}{2}\right] \\
& =\frac{\frac{\mathbf{a}^{A}-\mathbf{a}^{B}}{2}+\frac{\mathbf{c}^{A}-\mathbf{c}^{B}}{2}}{2}+\frac{\beta}{2(2+\beta)} \mathbf{b}\left(\mathbf{G}, \frac{2 \delta}{2+\beta},\left(\frac{\mathbf{a}^{A}-\mathbf{a}^{B}}{2}-\frac{\mathbf{c}^{A}-\mathbf{c}^{B}}{2}\right)\right) .
\end{aligned}
$$

Combing results in (F.3) and (F.4) yields

$$
\left\{\begin{array}{l}
\hat{\mathbf{p}}^{A}=\frac{\mathbf{a}^{A}+\mathbf{c}^{A}}{2}-\frac{\beta}{2(2-\beta)} \mathbf{b}\left(\mathbf{G}, \frac{2 \delta}{2-\beta},\left(\frac{\mathbf{a}^{A}+\mathbf{a}^{B}}{2}-\frac{\mathbf{c}^{A}+\mathbf{c}^{B}}{2}\right)\right)+\frac{\beta}{2(2+\beta)} \mathbf{b}\left(\mathbf{G}, \frac{2 \delta}{2+\beta},\left(\frac{\mathbf{a}^{A}-\mathbf{a}^{B}}{2}-\frac{\mathbf{c}^{A}-\mathbf{c}^{B}}{2}\right)\right) . \\
\hat{\mathbf{p}}^{B}=\frac{\mathbf{a}^{B}+\mathbf{c}^{B}}{2}-\frac{\beta}{2(2-\beta)} \mathbf{b}\left(\mathbf{G}, \frac{2 \delta}{2-\beta},\left(\frac{\mathbf{a}^{A}+\mathbf{a}^{B}}{2}-\frac{\mathbf{c}^{A}+\mathbf{c}^{B}}{2}\right)\right)-\frac{\beta}{2(2+\beta)} \mathbf{b}\left(\mathbf{G}, \frac{2 \delta}{2+\beta},\left(\frac{\mathbf{a}^{A}-\mathbf{a}^{B}}{2}-\frac{\mathbf{c}^{A}-\mathbf{c}^{B}}{2}\right)\right) .
\end{array}\right.
$$

Note that for the special case with $\mathbf{a}^{A}=\mathbf{a}^{B}=\mathbf{a}$ and $\mathbf{c}^{A}=\mathbf{c}^{B}=\mathbf{c}$, we must have $\hat{\mathbf{p}}^{A}=\hat{\mathbf{p}}^{B}$ by (F.4). Therefore, the equilibrium price is symmetric. Moreover, this common price vector equals

$$
\left[(2-\beta) \mathbf{I}_{n}-2 \delta \mathbf{G}\right]^{-1}\left[\left((1-\beta) \mathbf{I}_{n}-\delta \mathbf{G}\right) \mathbf{a}+\left(\mathbf{I}_{n}-\delta \mathbf{G}\right) \mathbf{c}\right]=\frac{\mathbf{a}+\mathbf{c}}{2}-\frac{\beta}{2}\left[(2-\beta) \mathbf{I}_{n}-2 \delta \mathbf{G}\right]^{-1}(\mathbf{a}-\mathbf{c})
$$

by (F.3), which is consistent with Theorem 1. Hence, we obtain the result in the theorem.

Proof of Theorem E.1: For the first result, note that $\mathbf{x}^{*}(\mathbf{G} ; \beta, \delta)=\mathbf{M}^{+}\left(\mathbf{a}-\mathbf{p}^{*}\right)$ by Corollary 1 . Plugging this formula for $\mathbf{p}^{*}$ in Theorem 1 and simplifying it yield the result. The second result follows from the fact $\Pi^{*}(\mathbf{G} ; \beta, \delta)=\left\langle\mathbf{p}^{*}-\mathbf{c}, \mathbf{x}^{*}\right\rangle$ and by straightforward calculation.

For the third result, we first compute the equilibrium payoff for player $i$ as follows:

$u_{i}^{*}:=\max _{x_{i}^{A}, x_{i}^{B}} a_{i}\left(x_{i}^{A}+x_{i}^{B}\right)-\left\{\frac{1}{2}\left(x_{i}^{A}\right)^{2}+\frac{1}{2}\left(x_{i}^{B}\right)^{2}+\beta x_{i}^{A} x_{i}^{B}\right\}+\delta \sum_{j=1}^{n} g_{i j} x_{i}^{A} x_{j}^{A}+\delta \sum_{j=1}^{n} g_{i j} x_{i}^{B} x_{j}^{B}-p_{i}^{A} x_{i}^{A}-p_{i}^{B} x_{i}^{B}$.

We can then plug into this expression the equilibrium prices $p_{i}^{A}=p_{i}^{A}=p_{i}^{*}$. Then, we use the first-order conditions in equilibrium to obtain:

$$
u_{i}^{*}=(1+\beta)\left(x_{i}^{*}\right)^{2} .
$$

The total consumer surplus is then given by:

$$
C S^{*}(\mathbf{G} ; \beta, \delta)=\sum_{i} u_{i}^{*}=\sum_{i}(1+\beta)\left(x_{i}^{*}\right)^{2}=(1+\beta)\left\langle\mathbf{x}^{*}, \mathbf{x}^{*}\right\rangle .
$$

The rest just follows from using the expressions of $\mathbf{x}^{*}(\mathbf{G} ; \beta, \delta)$ given in the theorem. To obtain the equilibrium total welfare $T W^{*}(\mathbf{G} ; \beta, \delta)$, we just need to add the consumer surplus and the profit. 